\title{
Speckle observations with PISCO in Calern (France): I. Astrometric measurements of visual binaries in 2015-2016.
}

\author{
M. Scardia ${ }^{1,2}$, J.-P. Rivet ${ }^{3}$, J.-L. Prieur ${ }^{4,5}$, L. Pansecchi ${ }^{1}$, R.W. Argyle ${ }^{6}$, J.F. Ling ${ }^{7}$, E. \\ Aristidi $^{3}$, A. Zanutta ${ }^{1}$, D. Vernet ${ }^{2}$, L. Abe ${ }^{3}$, P. Bendjoya ${ }^{3}$, C. Dimur ${ }^{\star \star \star}$, and O. Suarez ${ }^{2}$ \\ 1 INAF - Osservatorio Astronomico di Brera, Via E. Bianchi 46, 23807 Merate, Italy \\ 2 Université Côte d'Azur, Observatoire de la Côte d'Azur, France \\ 3 Université Côte d'Azur, Observatoire de la Côte d'Azur, CNRS, Laboratoire Lagrange, France \\ ${ }^{4}$ Université de Toulouse - UPS-OMP - IRAP, Toulouse, France \\ ${ }^{5}$ CNRS - IRAP, 14 avenue Edouard Belin, 31400 Toulouse, France \\ 6 Institute of Astronomy, Madingley Road, Cambridge, CB3 0HA, United Kingdom \\ 7 Observatorio Astrónomico R.M. Aller, Avda das Ciencias s/n. Departamento de Matemática Aplicada, \\ Universidad de Santiago de Compostela, 15782, Spain
}

Received June 13, 2019; accepted

Key words Stars: binaries: close - binaries: visual — astrometry — techniques: interferometric

We present relative astrometric measurements of visual binaries, made in 2015-2016 with the speckle camera PISCO at the 1-m Epsilon telescope of the C2PU facility (Observatoire de la Côte d'Azur, Calern site). Our observing list contains orbital couples as well as binaries whose motion is still uncertain. From our 1233 observations of 1173 multiple stars, we obtained 1170 new measurements with angular separations in the range $0^{\prime \prime} .1-8^{\prime \prime} .2$, and an average accuracy of $0^{\prime \prime} .0078$. The mean error on the position angles is $0^{\circ} .54$. Most of the position angles were determined without the usual $180^{\circ}$ ambiguity with the application of the direct vector autocorrelation technique and/or by inspection of the long integration files.

(C) 0000 WILEY-VCH Verlag GmbH \& Co. KGaA, Weinheim

${ }^{* *}$ Deceased October 28, 2017

\section{Introduction}

This paper presents the results of speckle observations of visual binary stars made in Calern (France) in 2015-2016 with the Pupil Interferometry Speckle camera and COronagraph (PISCO) on the 104-cm 'Epsilon' (East) telescope of the Centre Pédagogique Planètes et Univers (C2PU) facility (Bendjoya et al. 2012, https://www.oca.eu/fr/c2puaccueil, Lat $=43^{\circ} 45^{\prime} 13.2^{\prime \prime} \mathrm{N}$, Lon $=06^{\circ} 55^{\prime} 22.7^{\prime \prime} \mathrm{E}$ ). This telescope is located in the Calern site of the Côte d'Azur Observatory (OCA, France).

The purpose of this paper is to contribute to a better knowledge of the apparent relative motion of longperiod binaries so that very accurate orbits could be determined in the future and fundamental parameters such as stellar masses could be inferred from them. This papers opens a new series that will be the counterpart of the series of papers that have reported the results of observations with PISCO in Brera Astronomical Observatory (Merate, Italy): Scardia et al. 2005, 2006, 2007, 2008, Prieur et al. 2008, Scardia et al. 2009, Prieur et al. 2009, Scardia et al. 2010, Prieur et al. 2010, Scardia et al. 2011, Prieur et al. 2012, Scardia et al. 2013, Prieur et al. 2014, Scardia et al. 2015, Prieur et al. 2017, and Scardia et al. 2018, herein: Papers I to XVI. The focal instrument PISCO (Prieur et al., 1998) was developed at Observatoire Midi-Pyrénées (France) and first used at Pic du Midi observatory (France) from 1993 to 1998 . It was moved to Merate in 2003 and installed on the INAF Zeiss telescope that was dedicated to binary star observations until the first semester of 2015. In summer 2015, PISCO was moved to the Calern observing site of the Côte d'Azur Observatory in France. It was mounted on the Epsilon telescope and has been operated there since then. This paper presents the first observations made in Calern in 2015-2016.

In Sect. 2, we briefly describe our observations. In Sect. 3, we present and discuss the astrometric measurements. We also compare those measurements with the ephemerides computed with the published orbital elements, when available.

\section{Observations}

The observations were carried out with the Epsilon telescope in the Calern observing site (Fig.1) with the PISCO speckle camera equipped with the Andor iXon Ultra 897 back-illuminated EMCCD detector. We present in detail this detector in Section 2.3. PISCO was mounted on the side of the telescope (Fig.2) which allowed other experiments to be installed at 
the Cassegrain focus, when the telescope time was allocated to other observers. The procedure we used for observing binary stars with PISCO in Calern was the same as the one used in Merate (see Paper VI). For each observation, a series of about 10,000 shortexposure frames were processed in real-time with a PC running on Microsoft Windows 7 that was used for performing real-time processing with our program PiscoSpeck2. This program controlled the Andor camera, displayed the images and for each elementary image computed the mean auto-correlation with Worden's (1977) method (which subtracted most of the continuum), the mean power spectrum, the integration of the individual frames, the mean restricted triple correlation frame used for the quadrant determination (Aristidi et al. 1997) and the mean direct vector autocorrelation image which provided another way of obtaining this quadrant with the method proposed by Bagnuolo et al. (1992). Each set of 10,000 elementary frames was also recorded as a FITS cube on an internal SATA disk, for archiving and further processing if needed.

Our program PiscoSpeck2 had many parameters that could be changed by the user according to the observing conditions. We are giving here the usual values of some of those parameters. The Andor camera was used in the Electron-Multiplied mode with an exposure time of $20 \mathrm{~ms}$ and a cooling temperature of $-85^{\circ} \mathrm{C}$. Our program processed sets of 200 elementary frames of $256 \times 256$ pixels, which led to a field of $10^{\prime \prime} .8 \times 10^{\prime \prime} .8$ with the $20 \mathrm{~mm}$ eyepiece (see Table 1 ). To achieve realtime processing during the acquisition, the processing of one set of frames was done by one thread of our program, while another thread was acquiring the frames of the next set. Our observations required rather good seeing conditions, (i.e., FWHM seeing less than $2^{\prime \prime}$ ), and we added a selection of the best frames according to the seeing. Another selection criteria based on the mean value of the frame, was sometimes used to discard abnormal frames generated by the electronics. The background of each autocorrelation of index $k$ was estimated from the cross-correlation of the frame of index $k$ with the frame of index $k-4$. There was therefore a time delay of $80 \mathrm{~ms}$ between those two (uncorrelated) elementary frames, which led to a good estimation of the background (see Worden 1977) .

The scale of the eyepieces was calibrated with a grating mask placed on top of the telescope (see Fig. 1), as described in Paper III and Paper XII. This procedure allowed an absolute astrometric calibration of PISCO. The values found for the 10, 20, and $32 \mathrm{~mm}$ eyepieces are given in Table 1. In Calern we could use the metallic mask that was originally made for the 1-m Zeiss telescope in Merate, since the Epsilon telescope has the same diameter.
Table 1 Scale values obtained with a grating mask for the three magnifications that have been used in Table 2 .

\begin{tabular}{lll}
\hline $\begin{array}{l}\text { Eyepiece } \\
(\mathrm{mm})\end{array}$ & $\begin{array}{l}\text { scale } \\
(\text { mas/pixel })\end{array}$ & $\begin{array}{l}\text { error } \\
(\text { mas/pixel })\end{array}$ \\
\hline 10 & 18.01 & 0.14 \\
20 & 42.35 & 0.33 \\
32 & 89.86 & 0.66 \\
\hline
\end{tabular}

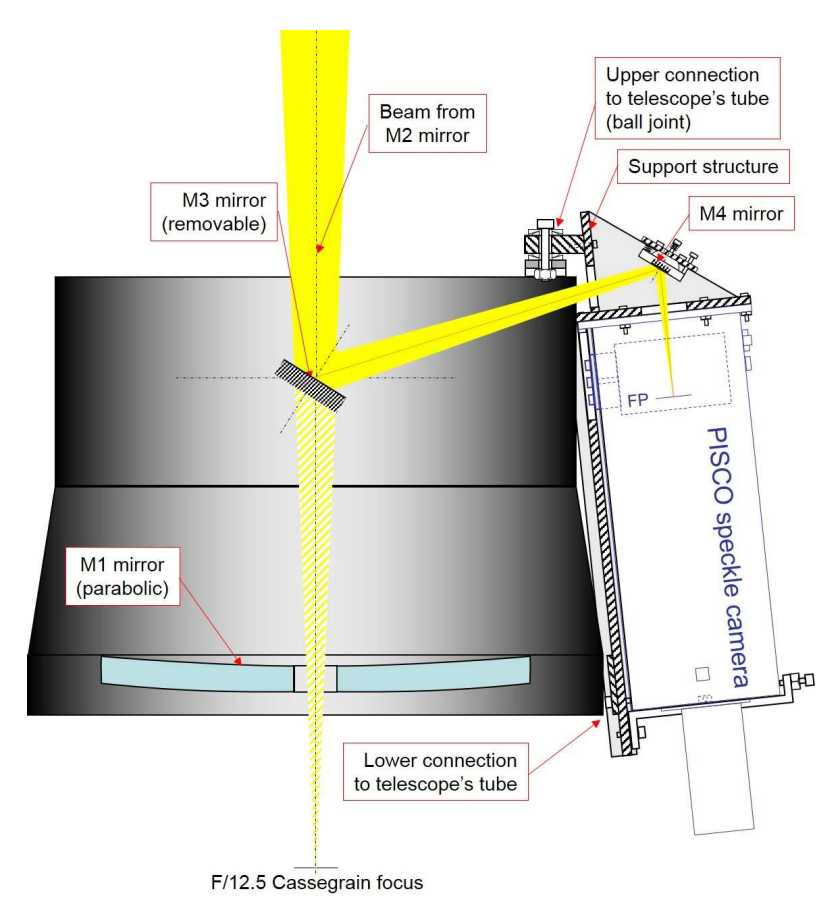

Fig. 2 PISCO was mounted on the side of the Epsilon telescope tube. The F/12.5 Cassegrain beam was deviated into PISCO through two flat mirrors M3 and M4. If needed, the M3 mirror could be removed to give access to the Cassegrain beam for other observations.

\subsection{Observing list}

Our observing list basically includes all the visual binaries from the Washington Double Star Catalog (Mason et al. 2019, hereafter WDS catalog) that require new measurements to improve their orbits, and that meet the technical constraints of our setup (declination range, magnitude, magnitude difference, and angular separation).

Compared to the Merate site, the new site in Calern is better, with a higher rate of observable nights (about twice as much). During the night, the artificial lighting is considerably reduced in Calern and the sky background is much darker, which allow us to observe fainter objects. The gain in air transparency is also explained by the higher altitude of Calern, which is $1270 \mathrm{~m}$ above the sea level, compared to the altitude of $320 \mathrm{~m}$ of Merate.

The EMCCD detector that we now use with PISCO is more sensitive than the ICCD detector we used in Merate. This leads to a substantial gain in sensitivity 

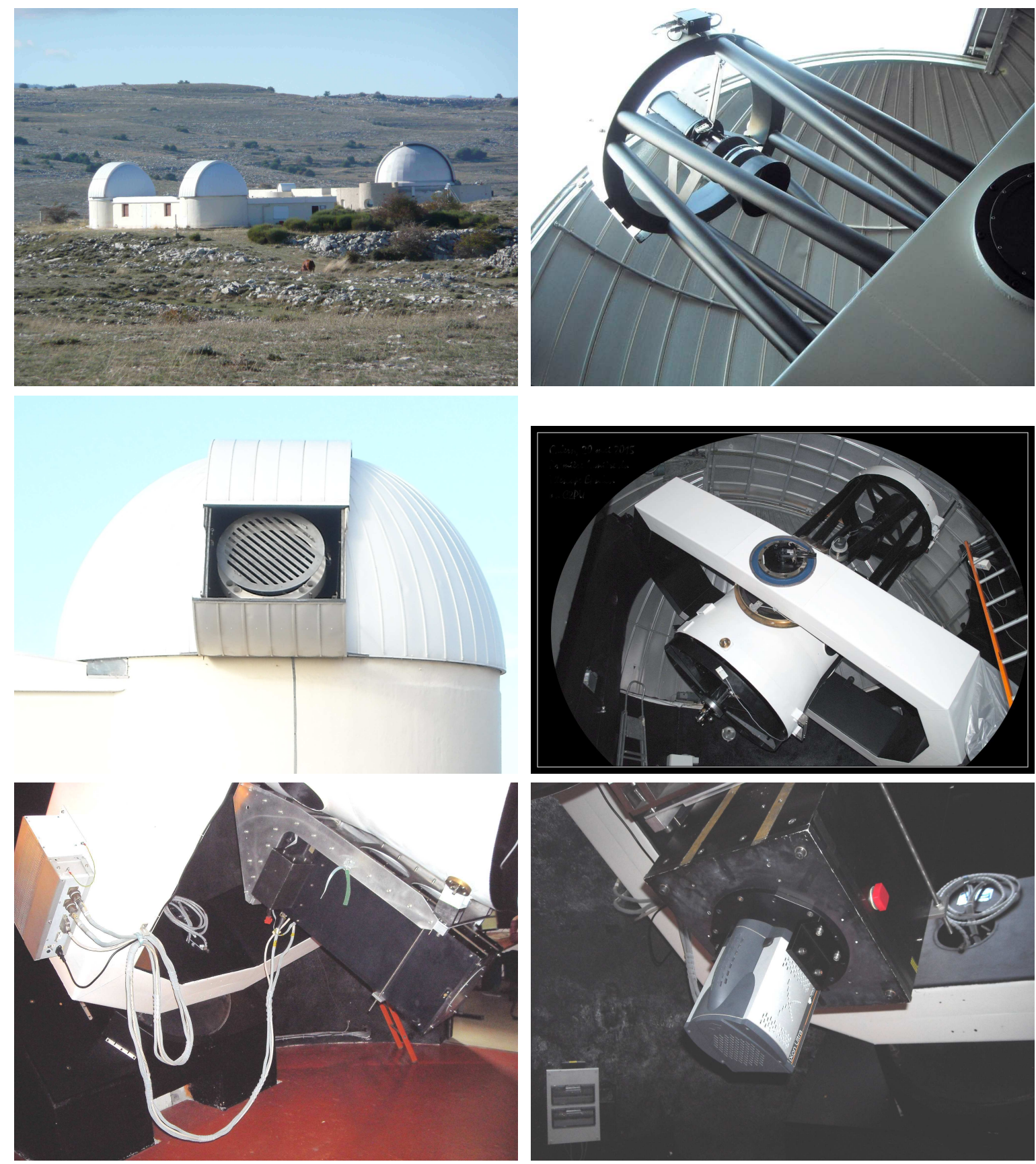

Fig. 1 Photographs of PISCO mounted on the 1-m Epsilon telescope in Calern. From top to bottom and left to right: overview of the observatory (the Epsilon telescope dome is the central one), upper part of the telescope, grating mask fitted on top of the telescope during scale calibration, telescope before the installation of the instrument (May 2015), PISCO mounted on the side of the telescope tube, EMCCD Andor iXon Ultra 897 camera attached to PISCO.

and the limiting magnitude is now around $V=13$ mag (see Fig. 4b), which is about three magnitudes fainter than in Merate. The equatorial yoke mount of the Epsilon telescope has unfortunately some constraints that were not present with the equatorial German mount of the Zeiss telescope. For instance, objects close to the Northern pole with a declination north of $60^{\circ}$ cannot be observed with the Epsilon telescope (see Fig. 3). The atmospheric seeing becomes seriously degraded at low heights and generally prevents observations of objects with declinations south of $-5^{\circ}$ (although objects with smaller declination, down to $-10^{\circ}$ have been observed in good seeing conditions).

Our sample consists of visual binaries with the following characteristics, that are linked to instrumental or atmospheric limitations:

- declination north of $-5^{\circ}$ and south of $60^{\circ}$,

- brighter than 13th magnitude in $V$, 


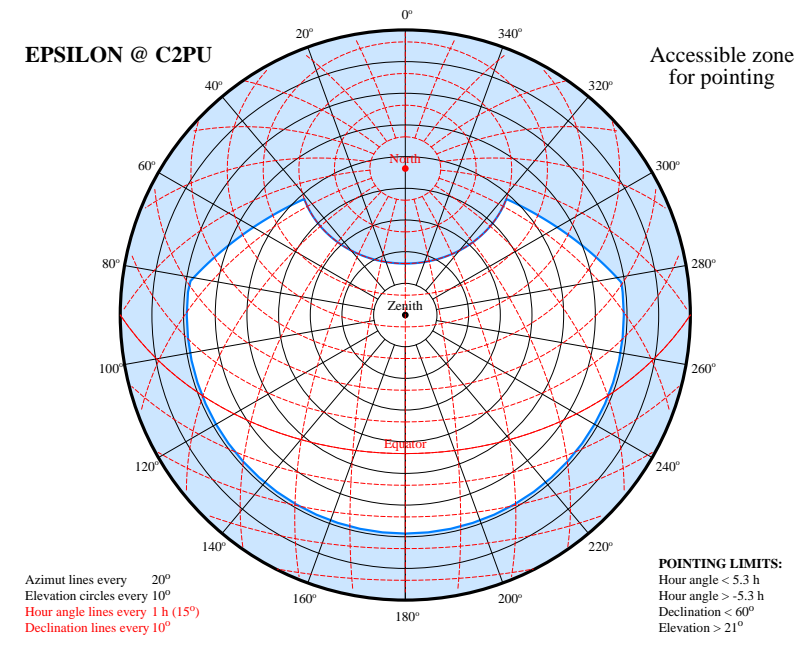

Fig. 3 Azimuthal boundaries of the targets imposed by the equatorial yoke mount of the Epsilon telescope.

- magnitude difference less than 4 ,

- angular separation smaller than $\approx 8^{\prime \prime}$.

- angular separation larger than $0 .{ }^{\prime \prime} 13$.

The last two limitations were chosen so that the binary systems fit inside the field of the camera and that their separation is larger than the diffraction limit of the telescope. The closest separation that can be measured with PISCO in Calern is of the order of this diffraction limit, which is $\rho_{d}=\lambda / D=0^{\prime \prime} .13$ with the $R$ filter (i.e. $\lambda=650 \mathrm{~nm})$ and the Epsilon telescope whose diameter is $D=1.04 \mathrm{~m}$. Our targets are taken from the WDS catalog data base when imposing the limitations given above. Our sample thus contains of a few thousands objects.

\subsection{Statistics on our observations made in 2015-2016}

The distribution of the angular separations measured in this paper is displayed in Fig. 4a and shows a maximum for $\rho \approx 0^{\prime \prime} .6$. The largest separation of $8^{\prime \prime} .2$ was obtained for $03579+5142$ SCA176BC. The smallest separation was measured for $15009+5745$ MLR 602 with $\rho=0^{\prime \prime} .130$, which is very close to the diffraction limit of $\rho_{d}=0^{\prime \prime} .13$ for the Epsilon telescope and the $R$ filter.

The distribution of the apparent magnitudes $m_{V}$ and of the difference of magnitudes $\Delta m_{V}$ between the two components are plotted in Figs. 4b and 4c, respectively. The telescope aperture and detector sensitivity led to a limiting magnitude of $m_{V} \approx 13$ (Fig. $4 \mathrm{~b}$ ) and a maximum $\Delta m_{V}$ for speckle measurements of about 3.7 (Fig. 4c).

Using the Hipparcos parallaxes, we were able to construct the HR diagram of those binaries, which is displayed in Fig. 5. Out of the 491 objects whose parallax was measured by Hipparcos, we only plotted the

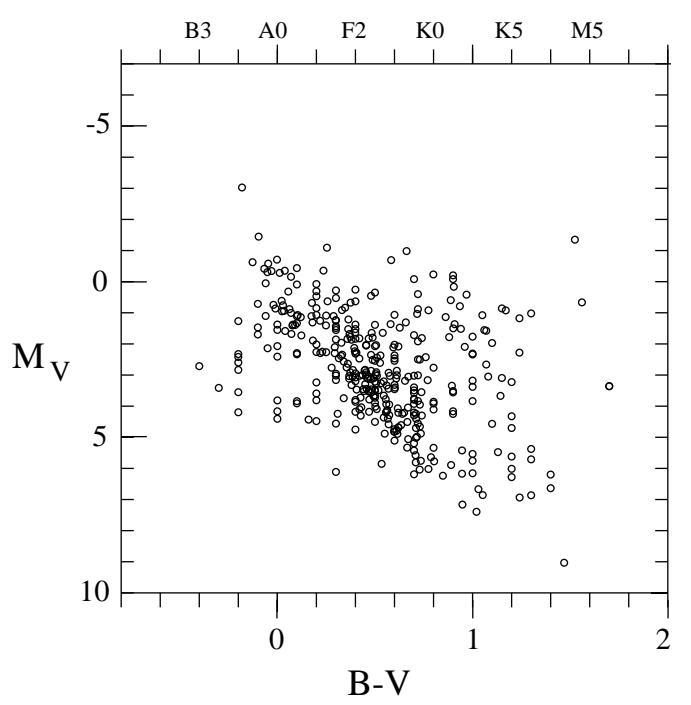

Fig. 5 HR diagram of the binaries measured in Table 2, for which the Hipparcos parallaxes have a relative error smaller than $50 \%$ (i.e., 378 objects).

378 objects for which the relative uncertainty on the Hipparcos parallax was smaller than $50 \%$.

\subsection{The EMCCD Andor iXon Ultra 897 camera}

The camera we have used for our observations is a back-illuminated Andor iXon Ultra 897, with a sensor format of $512 \times 512$ and $16 \times 16 \mu \mathrm{m}$ square pixels. It uses the Electron Multiplying CCD ultra-sensitive technology, which is well adapted to very low light intensities. Its low read-out noise allows single photon detection. The theoretical quantum efficiency of the chip is shown in Fig. 6. It is much better than the ICCD camera that we used in Merate from 2004 to 2015, whose quantum efficiency was about $6 \%$. The corresponding gain in sensitivity is about of $15 \mathrm{x}$, which corresponds to $2.5 \times \log (15) \approx 3$ mag. Indeed, we experimentally confirmed this expected gain: the limiting magnitude of our instrumentation increased from $V=10$ in Merate to $V=13$ in Calern.

The Andor iXon Ultra 897 is fitted with a four-stage Peltier cooling assembly which utilizes the thermoelectric effect (TE) to rapidly cool the sensor down to the stable operating temperature. TE coolers have a cold end (in contact with the sensor) and a hot end where heat must be efficiently dissipated for effective cooling of the sensor. Although water cooling is possible with that camera and would be more effective, we use a simpler (and cheaper) system, with forced air cooling (in-built fan) which allow us to operate the sensor with a cooling temperature of $-85^{\circ} \mathrm{C}$. The iXon Ultra 

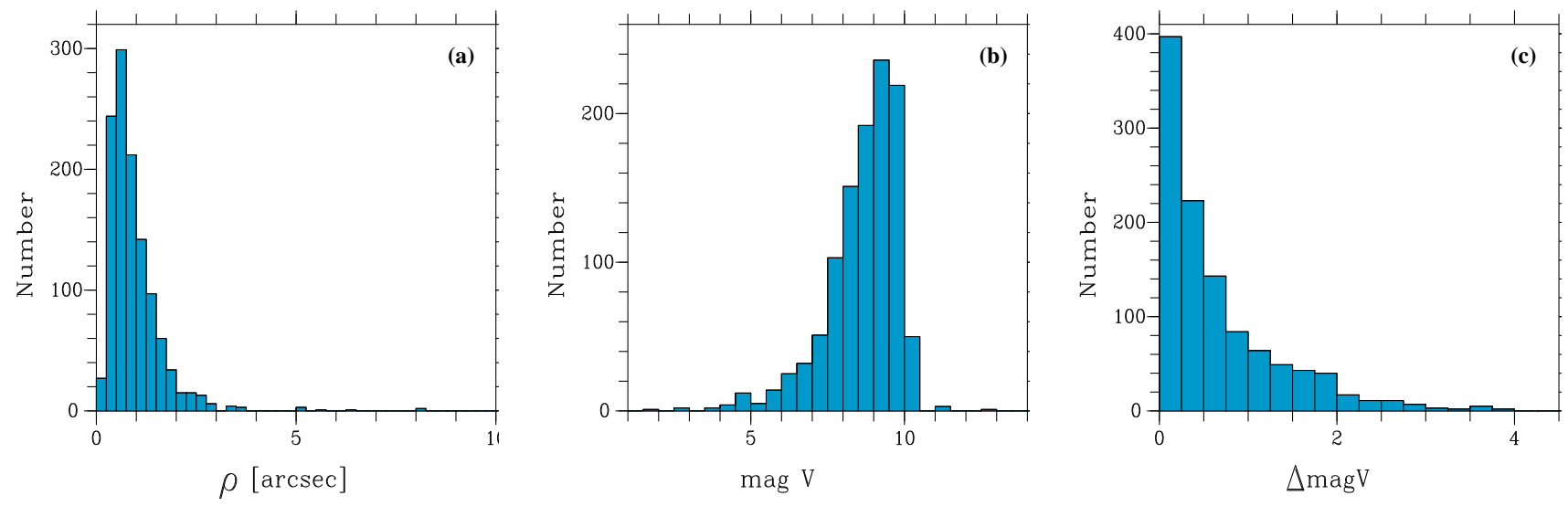

Fig. 4 Distribution of the angular separations of the 1170 measurements of Table 2 in (a), of the total visual magnitudes of the corresponding binaries in (b) and of the differences of magnitude between their two components in (c).

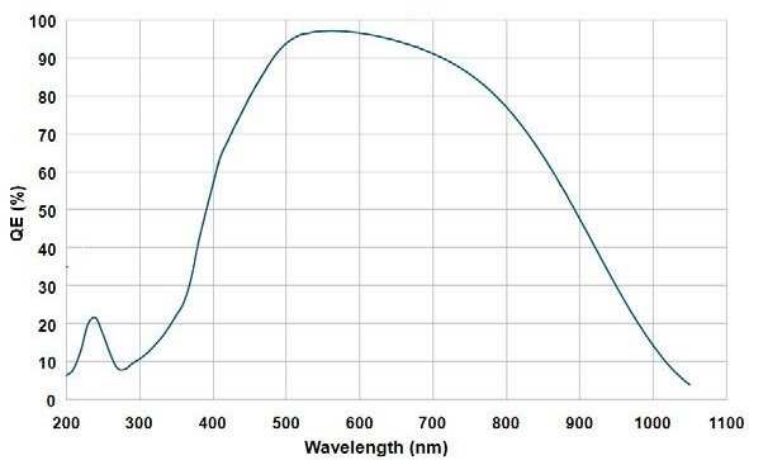

Fig. 6 Quantum efficiency of the back-illuminated EMCCD Andor iXon Ultra 897 (from the Andor user manual).

camera also contains temperature control components, which regulate the cooling of the camera and ensure that a stable temperature is maintained between and throughout measurements. We have found this regulation very effective for reaching the set point in a few minutes only, and very stable during the observations with a variation of temperature less than $0.1^{\circ} \mathrm{C}$.

The sensor has a frame transfer architecture. The frame-transfer EMCCD uses a two-part sensor in which one-half of the array is used as a storage region and is protected from light by a light-tight mask. Incoming photons are allowed to fall on the uncovered portion of the array and the accumulated charge is then rapidly shifted into the masked storage region for transfer to the serial output register. While the signal is being integrated on the light-sensitive portion of the sensor, the stored charge is read out. Frame transfer devices have typically faster frame rates than full frame devices, and have the advantage of a high duty cycle i.e. the sensor is always collecting light.

Essentially, the EMCCD sensor is capable of detecting single photon events without an image inten- sifier, achievable by way of a unique electron multiplying structure built into the chip. Traditional CCD cameras offered high sensitivity, with readout noises in single figure $<10 e-$ but at the expense of slow readout. Hence they were often referred to as 'slow scan' cameras. The fundamental constraint came from the CCD charge amplifier. To have high speed operation the bandwidth of the charge amplifier needs to be as wide as possible. However, it is a fundamental principle that the noise scales with the bandwidth of the amplifier, hence higher speed amplifiers have higher noise. Slow scan CCD's have relatively low bandwidth and hence can only be read out at modest speeds, typically less than 1MHz. EMCCD cameras avoid this constraint by amplifying the charge signal before the charge amplifier and hence maintain unprecedented sensitivity at high speeds. By amplifying the signal the readout noise is effectively by-passed and, as such, EMCCD readout noise is no longer a limit on sensitivity (and can often be considered negligible).

We have developed a program, PiscoSpeck2, in $\mathrm{C}++$ with the Widget graphic library that allows full control of most of the functions of this camera and performs real-time processing of the elementary frames. It has similar functionality and user-interface as the program vcrb that we had written for the ICCD mounted on PISCO in Merate and that we have used there for more than ten years. Although PiscoSpeck2 was designed for the Andor iXon Ultra 897 camera which has a serial USB2 interface, our new program is generic and has been successfully tested and used with different EMCCD cameras, such as the Andor LUCA and Andor DV885 that we employ for observing binaries with the OCA $76 \mathrm{~cm}$-refractor (see Gili et al., 2014). It can also be used for processing FITS files obtained with other cameras and can be easily installed on any 64 -bit 
Windows PC (please contact us if you are interested in using PiscoSpeck2).

\section{Astrometric measurements}

The 1233 observations made in 2015-2016 with PISCO at Calern Observatory are presented in Table 2. They concern 1173 visual binaries or multiple objects. For each object, we report its WDS name in Col. 1, the official double star designation in Col. 2 (sequence is "discoverer-number"), and the ADS number in Col. 3 (Aitken, 1932) when available. For each observation, we then give the epoch in Besselian years (Col. 4), the filter (Col. 5), the focal length of the eyepiece used for magnifying the image (Col. 6), the angular separation $\rho$ (Col. 7) with its error (Col. 8) in arcseconds, and the position angle $\theta$ (Col. 9) with its error (Col. 10) in degrees. In Col. 11, we report some notes and some information about the secondary peaks of the autocorrelation files (e.g. diffuse, faint or elongated) or about the power spectrum (NF: no fringes). The mention "artifacts" was used when some spurious noise horizontal pattern was present on the autocorrelations. This was caused by the detector noise at low levels.

We had to face some technical problems in 2015, when setting up the new configuration of PISCO in Calern (e.g. when focusing the eyepieces). This explains why some measurements in separation are missing in Table 2 for some of the first measurements made in 2015, because they appeared to be unreliable. Those problems slightly changed the magnification scale only, and had no implications on the position angle measurements.

For the systems with a known orbit, the $(O-C)$ (Observed minus Computed) residuals of the $\rho$ and $\theta$ measurements are displayed in Cols. 13 and 14, respectively. The corresponding authors are given in Col. 12, using the bibliographic style of the "Sixth Catalog of Orbits of Visual Binary Stars" (Hartkopf \& Mason, 2019, hereafter OC6).

When not explicitly specified, the measurements refer to the $\mathrm{AB}$ components of those systems. We precise 'AB' when that the system is multiple and known to have other components. In Col. 14, an exponent ${ }^{Q}$ is appended to the values for which a quadrant inconsistency was found between our measures and the positions derived from the orbital elements published for this object.

The characteristics of the $\mathrm{R}$ and RL filters used for obtaining those measurements are given in Table 2 of Paper XII. Some objects were observed without any filter because they were too faint. This is indicated with W (for "white" light) in the filter column (Col. 5 of Table 2). In that case, the bandpass and central wavelength correspond to that of the EMCCD Andor detector (see Fig 6).
As for the other papers of the Merate observations series, position measurements were obtained by an interactive processing of the autocorrelation files computed in real time during the observations. This processing led to a series of measurements with different background estimates and simulated noise, from which we derived the mean values and the standard deviation of those multiple measurements (see Paper III for more details). The final measures and their errors are displayed in Table 2. The average error values of the measurements reported in this table are $\left\langle\sigma_{\rho}\right\rangle=0^{\prime \prime} .0078 \pm 0^{\prime \prime} .0049$ and $\left\langle\sigma_{\theta}\right\rangle=0^{\circ} .54 \pm 1^{\circ} .41$, for the angular separations and the position angles, respectively.

\subsection{Quadrant determination}

As our astrometric measurements were obtained from the symmetric autocorrelation files, the $\theta$ values first presented a $180^{\circ}$ ambiguity. To resolve this ambiguity and determine the quadrant containing the companion, we used the "direct vector autocorrelation" (DVA) method proposed by Bagnuolo et al (1992) which is now implemented in our new real-time processing software. The other method which is also implemented in our software is the "restricted triple correlation" (RTC) of Aristidi et al. (1997), which was widely used in Merate with our old Intensified CCD camera. In this paper we prefer not using it, since the RTC often led to wrong results with that data, even for wide binaries. The reason may be due to low light levels and photon noise effects. Conversely, we used the DVA method that led to better results with the observational data presented here. Of course, for the couples with the largest separations, a straightforward quadrant determination was done when the companions could be directly spotted on the long integration files.

As a result, in Table 2, we have been able to give the unambiguous (i.e. "absolute") position angles of 454 out of 1170 measurements, i.e. $39 \%$ of the total. They are marked with an asterisk in Col 9. When our quadrant determination procedure failed, the angular measurement was reduced to the quadrant reported in the WDS catalog, which is extracted from the Fourth Catalog of Interferometric Measurements of Binary Stars (Hartkopf et al. 2018, hereafter IC4). For HU1267, our two observations of April 20th and May 2nd 2016, clearly show that the brightest component was not in the same quadrant (see Fig. 7), which may be an indication that one of the components is a variable star.

In Table 3, we display some information about the objects for which our "absolute" $\theta$ values are not consistent with the values tabulated in the WDS. In Col. 3, we indicate the quadrant $(\mathrm{Q})$ that we obtained from our observations, using the usual convention of numbering it from 1 to 4 to indicate the North-East, South-East, South-West and North-West quadrants, respectively. In 
Table 2 Table of speckle measurements and O-C residuals with published orbits (begin.)

\begin{tabular}{|c|c|c|c|c|c|c|c|c|c|c|c|c|c|}
\hline WDS & Name & ADS & Epoch & Fil. & $\begin{array}{l}\text { Eyep. } \\
(\mathrm{mm})\end{array}$ & $\begin{array}{c}\rho \\
\left({ }^{\prime \prime}\right)\end{array}$ & $\begin{array}{l}\sigma_{\rho} \\
\left({ }^{\prime \prime}\right)\end{array}$ & $\begin{array}{c}\theta \\
\left({ }^{\circ}\right)\end{array}$ & $\begin{array}{l}\sigma_{\theta} \\
\left(^{\circ}\right)\end{array}$ & Notes & Orbit & $\begin{array}{r}\Delta \rho(\mathrm{O}-\mathrm{C}) \\
\left({ }^{\prime \prime}\right)\end{array}$ & $\begin{array}{r}\Delta \theta(\mathrm{O}-\mathrm{C}) \\
\left({ }^{\circ}\right)\end{array}$ \\
\hline $00013+3351$ & TDS1248 & - & 2016.715 & $\mathrm{~W}$ & 20 & - & - & - & - & Unres. & & & \\
\hline $00013+3351$ & TDS1248 & - & 2016.677 & $\mathrm{~W}$ & 20 & - & - & - & - & Unres. & & & \\
\hline $00014+3937$ & HLD60 & 17178 & 2015.940 & $\mathrm{R}$ & 20 & 1.331 & 0.015 & $166.0^{*}$ & ${ }^{*} 0.3$ & & Hrt2011a & 0.03 & -1.2 \\
\hline $00015+3044$ & HO208 & 17167 & 2016.035 & $\mathrm{R}$ & 20 & 1.108 & 0.006 & $185.6^{*}$ & ${ }^{*} 0.3$ & Elongated & & & \\
\hline $00026+5534$ & A1499 & 3 & 2016.854 & $\mathrm{R}$ & 20 & 1.560 & 0.008 & $208.4^{*}$ & ${ }^{*} 0.3$ & & & & \\
\hline $00028+0208$ & BU281AB & 9 & 2016.879 & $\mathrm{R}$ & 20 & 1.565 & 0.008 & $159.8^{*}$ & ${ }^{*} 0.3$ & & $\operatorname{Lin} 2015 a$ & 0.01 & 0.2 \\
\hline $00029+4715$ & A 800 & 10 & 2015.915 & $\mathrm{R}$ & 32 & 1.653 & 0.035 & 113.2 & 0.4 & & & & \\
\hline $00029+4715$ & A 800 & 10 & 2015.940 & $\mathrm{R}$ & 20 & 1.620 & 0.015 & $113.8^{*}$ & ${ }^{*} 0.3$ & Elongated & & & \\
\hline $00037+5329$ & WOR30 & - & 2016.854 & $\mathrm{R}$ & 20 & 0.851 & 0.006 & $181.9^{*}$ & ${ }^{*} 0.5$ & & & & \\
\hline $00039+2759$ & $\mathrm{~A} 429 \mathrm{AB}$ & 24 & 2015.920 & $\mathrm{R}$ & 20 & 0.546 & 0.006 & 329.6 & 0.3 & & & & \\
\hline $00048+3810$ & BU862 & 34 & 2015.940 & $\mathrm{R}$ & 20 & 0.878 & 0.015 & $26.6^{*}$ & ${ }^{*} 0.5$ & & Cou1986b & 0.04 & -1.7 \\
\hline $00048+3810$ & BU862 & 34 & 2016.650 & $\mathrm{R}$ & 20 & 0.883 & 0.004 & 27.8 & 0.4 & & Cou1986b & 0.04 & -0.8 \\
\hline $00112+3331$ & COU648 & - & 2015.920 & $\mathrm{~V}$ & 20 & 0.425 & 0.008 & 308.2 & 0.6 & & & & \\
\hline $00112+3331$ & COU648 & - & 2015.920 & $\mathrm{R}$ & 20 & 0.424 & 0.007 & 308.4 & 0.4 & & & & \\
\hline $00115+2936$ & HO1 & 142 & 2016.704 & $\mathrm{R}$ & 20 & 1.259 & 0.010 & 157.9 & 0.3 & & & & \\
\hline $00134+2659$ & STT2AB & 161 & 2015.915 & $\mathrm{R}$ & 20 & 0.431 & 0.025 & 156.3 & 0.6 & & Hrt2008 & 0.02 & 1.5 \\
\hline $00134+2659$ & STT2AB & 161 & 2015.915 & $\mathrm{R}$ & 20 & 0.436 & 0.025 & 156.9 & 0.7 & & Hrt2008 & 0.02 & 2.1 \\
\hline $00149+5315$ & A903 & 186 & 2015.920 & $\mathrm{~V}$ & 20 & 0.943 & 0.010 & 134.0 & 0.3 & & & & \\
\hline $00151+1907$ & A2202 & 196 & 2016.704 & $\mathrm{R}$ & 20 & 0.626 & 0.007 & 154.2 & 0.3 & Elongated & & & \\
\hline $00187+1559$ & STF25AB & 257 & 2016.704 & $\mathrm{R}$ & 20 & 1.052 & 0.005 & 195.5 & 0.4 & & & & \\
\hline $00209+1059$ & BU1093 & 287 & 2016.879 & $\mathrm{R}$ & 20 & 0.777 & 0.017 & $119.1^{*}$ & * 0.8 & & Lin $2010 c$ & 0.01 & -0.5 \\
\hline $00211+3539$ & HU1202 & 291 & 2016.879 & $\mathrm{R}$ & 20 & 1.101 & 0.025 & $195.3^{*}$ & * 0.8 & Elongated & & & \\
\hline $00220+4033$ & A1502 & 301 & 2016.879 & $\mathrm{R}$ & 20 & 0.942 & 0.016 & $245.3^{*}$ & * 1.6 & & & & \\
\hline $00256+3629$ & HO210 & 344 & 2016.879 & $\mathrm{R}$ & 20 & 0.962 & 0.010 & $77.6^{*}$ & * 0.3 & Diffuse & & & \\
\hline $00297+5855$ & A909AB & 399 & 2016.854 & $\mathrm{R}$ & 20 & 0.998 & 0.005 & $40.9^{*}$ & ${ }^{*} 0.3$ & & & & \\
\hline $00335+4006$ & HO3 & 463 & 2016.876 & $\mathrm{R}$ & 20 & 0.399 & 0.004 & $141.1^{*}$ & ${ }^{*} 0.3$ & & Tok2017a & 0.02 & -0.3 \\
\hline $00344+1844$ & COU250 & - & 2016.876 & $\mathrm{R}$ & 20 & 1.305 & 0.007 & $39.0^{*}$ & ${ }^{*} 0.3$ & & & & \\
\hline $00351+0209$ & RST5182 & - & 2016.876 & $\mathrm{R}$ & 20 & 1.415 & 0.007 & 166.9 & 0.3 & & & & \\
\hline $00357+3429$ & HU1011 & 492 & 2016.857 & $\mathrm{R}$ & 20 & 0.339 & 0.009 & 81.7 & 1.8 & Elongated & Ole2002a & -0.08 & -7.4 \\
\hline $00364+1213$ & A 807 & 506 & 2016.876 & $\mathrm{R}$ & 20 & 0.835 & 0.004 & $233.4^{*}$ & * 0.3 & & & & \\
\hline $00364+5621$ & A913 & 501 & 2016.857 & $\mathrm{R}$ & 20 & 0.711 & 0.004 & 89.9 & 0.7 & & & & \\
\hline $00366+5609$ & A914 & 504 & 2016.718 & $\mathrm{R}$ & 20 & 0.453 & 0.004 & 21.0 & 0.7 & & $\operatorname{Lin} 2007 c$ & 0.03 & 1.0 \\
\hline $00367+3814$ & A1506 & 511 & 2016.718 & $\mathrm{R}$ & 20 & 1.223 & 0.006 & 19.9 & 0.3 & & & & \\
\hline $00378+4915$ & HU512 & 522 & 2016.879 & $\mathrm{R}$ & 20 & 0.918 & 0.009 & 168.2 & 0.3 & & & & \\
\hline $00391+5128$ & HU513 & 545 & 2016.718 & $\mathrm{R}$ & 20 & 0.987 & 0.005 & 210.7 & 0.3 & Elongated & & & \\
\hline $00440+4658$ & A652 & 614 & 2016.718 & $\mathrm{R}$ & 20 & 0.349 & 0.012 & 163.0 & 0.7 & & & & \\
\hline $00508+3203$ & $\mathrm{~A} 922 \mathrm{Aa}, \mathrm{Ab}$ & 691 & 2016.873 & $\mathrm{R}$ & 20 & 0.575 & 0.010 & 335.5 & 0.4 & Elongated & Msn2001c & 0.08 & -5.1 \\
\hline $00525+1740$ & A2306 & 715 & 2016.721 & $\mathrm{R}$ & 20 & 1.395 & 0.007 & 302.1 & 0.3 & & & & \\
\hline $00542+5108$ & HU1018 & 739 & 2015.940 & $\mathrm{R}$ & 20 & 0.936 & 0.015 & 58.2 & 0.3 & & & & \\
\hline $00544+5432$ & A1258 & 741 & 2015.942 & $\mathrm{R}$ & 20 & 0.568 & 0.015 & $200.4^{*}$ & ${ }^{*} 0.3$ & Elongated & & & \\
\hline $00546+1911$ & STT20AB & 746 & 2015.942 & $\mathrm{R}$ & 20 & 0.611 & 0.015 & 176.4 & 0.3 & Elongated & Doc2014a & 0.02 & -0.6 \\
\hline $00549+1928$ & A2208 & 756 & 2016.721 & $\mathrm{R}$ & 20 & 1.553 & 0.008 & 91.5 & 0.3 & & & & \\
\hline $00554+3040$ & BU500 & 768 & 2015.942 & $\mathrm{R}$ & 20 & 0.477 & 0.015 & 122.4 & 0.3 & & & & \\
\hline $00587+4457$ & A925 & 808 & 2016.876 & $\mathrm{R}$ & 20 & 1.099 & 0.005 & $106.8^{*}$ & ${ }^{*} 0.3$ & & & & \\
\hline $01004+1803$ & BRT1927 & - & 2015.942 & $\mathrm{R}$ & 20 & 1.927 & 0.015 & $170.3^{*}$ & ${ }^{*} 0.3$ & Elon & & & \\
\hline $01006+4719$ & MAD1 & 829 & 2015.942 & $\mathrm{R}$ & 20 & 0.840 & 0.015 & 0.3 & 0.3 & Elongated & Lin2012a & 0.08 & 1.3 \\
\hline $01010+4830$ & COU2256 & - & 2016.726 & $\mathrm{R}$ & 20 & 0.575 & 0.008 & 126.5 & 0.3 & & & & \\
\hline $01014+1155$ & BU867 & 828 & 2015.942 & $\mathrm{R}$ & 20 & 0.675 & 0.015 & $351.8^{*}$ & ${ }^{*} 0.4$ & Elongated & Hrt2008 & 0.06 & -0.7 \\
\hline $01021+3658$ & TDS1731 & - & 2016.726 & $\mathrm{R}$ & 20 & 0.772 & 0.011 & 235.6 & 0.3 & Uncertain & & & \\
\hline $01041+2635$ & COU351 & - & 2016.033 & $\mathrm{R}$ & 20 & 0.804 & 0.005 & $243.5^{*}$ & ${ }^{*} 0.3$ & Elongated & & & \\
\hline $01047+0029$ & RST4744 & - & 2016.726 & $\mathrm{R}$ & 20 & 0.423 & 0.009 & 104.3 & 0.5 & & & & \\
\hline $01055+2107$ & AG14 & 896 & 2016.873 & $\mathrm{R}$ & 20 & 0.810 & 0.012 & 309.6 & 0.4 & Elongated & Fmr2014f & -0.03 & -0.5 \\
\hline $01070+3014$ & A929AB & 887 & 2016.942 & $\mathrm{R}$ & 20 & 0.672 & 0.007 & $307.7^{*}$ & * 1.0 & Elongated & & & \\
\hline $01071+4744$ & A931 & 916 & 2016.942 & $\mathrm{R}$ & 20 & - & - & - & - & Unres. & & & \\
\hline
\end{tabular}

Note: In column 9 , the exponent ${ }^{*}$ indicates that the position angle $\theta$ could be determined without the $180^{\circ}$ ambiguity. 
Table 3 Discrepant quadrant measures with WDS catalog. The difference of magnitude between the two components, from the Hipparcos and Tycho Catalogues (ESA, 1997) is reported in Col. 5.

\begin{tabular}{llccl}
\hline WDS & Name & $\mathrm{Q}$ & Filter & $\Delta m_{V}$ \\
\hline $01070+3014$ & A929AB & 4 & $\mathrm{R}$ & 0.08 Tycho \\
$01365+4336$ & ES1495 & 1 & $\mathrm{R}$ & 0.11 Tycho \\
$01509+4037$ & COU1361 & 4 & $\mathrm{~W}$ & - \\
$03279+4551$ & COU1687 & 2 & $\mathrm{R}$ & 0.20 Tycho \\
$03354+3529$ & POP83 & 1 & $\mathrm{R}$ & 0.57 Tycho \\
$03584+5645$ & TDS2700 & 3 & $\mathrm{R}$ & 0.04 Tycho \\
$04123+0939$ & STT74 & 4 & $\mathrm{R}$ & - \\
$05065+4347$ & COU2465 & 3 & $\mathrm{R}$ & 0.02 Tycho \\
$06318+2124$ & TDS254 & 2 & $\mathrm{R}$ & 0.05 \\
$10192+2034$ & STF1423 & 2 & $\mathrm{R}$ & 0.68 Tycho \\
$10382+2636$ & STF1454 & 2 & $\mathrm{R}$ & 1.96 Tycho \\
$11080+2659$ & TDS7688 & 1 & $\mathrm{R}$ & 0.07 Tycho \\
$11098+1009$ & A2775 & 2 & $\mathrm{R}$ & 1.28 Tycho \\
$11468+1500$ & BU602 & 4 & $\mathrm{R}$ & 1.82 Tycho \\
$12093+1525$ & A2056 & 2 & $\mathrm{R}$ & 0.36 Tycho \\
$12111+1228$ & HDS1719 & 4 & $\mathrm{R}$ & 0.08 Tycho \\
$12120+1300$ & A1780 & 2 & $\mathrm{R}$ & 1.98 Tycho \\
$12167+3004$ & AG176 & 1 & $\mathrm{R}$ & 0.00 Tycho \\
$12194+1744$ & A2059 & 3 & $\mathrm{R}$ & 1.70 Hipparcos \\
$12340+2650$ & COU595 & 1 & $\mathrm{R}$ & 0.92 Tycho \\
$13041+5227$ & MLR704 & 3 & $\mathrm{R}$ & 0.53 Hipparcos \\
$13222+2710$ & A565 & 3 & $\mathrm{R}$ & 0.43 Tycho \\
$13223+2631$ & A566 & 3 & $\mathrm{R}$ & 0.92 Tycho \\
$14122+4411$ & STT278 & 2 & $\mathrm{R}$ & 0.33 Hipparcos \\
$14188+5934$ & HU1267 & $1-3$ & $\mathrm{R}$ & 0.12 Tycho \\
$14333+2725$ & A688 & 3 & $\mathrm{R}$ & 0.26 Tycho \\
$15100+2807$ & TDS768 & 3 & $\mathrm{R}$ & 0.45 Tycho \\
$15368-0438$ & RST4545 & 2 & $\mathrm{R}$ & 1.24 Hipparcos \\
$20238+4146$ & COU2641 & 2 & $\mathrm{R}$ & 0.02 Tycho \\
$21555+5232$ & STT456AB & 3 & $\mathrm{R}$ & 0.68 Tycho \\
\hline & & & & \\
& & &
\end{tabular}

Col. 4 we indicate which filter we have used ( $W$ indicates the absence of filter). We report the difference of magnitude between the two components from the IC4 in Col. 5. For those objects, the small value or the uncertainty in of the magnitude difference $\Delta m_{V}$ may account for the discrepancy. For many others, the small number of published observations may account for this discrepancy. However, since our determination is based on statistical optics, we cannot rule out some spurious results due to statistical noise.

\subsection{Comparison with published ephemerides}

The $(O-C)$ (Observed minus Computed) residuals of the measurements for the systems with a known orbit in Table 2 are displayed in Cols. 13 and 14 for the separation $\rho$ and position angle $\theta$, respectively. Those residuals were obtained with a selection of valid orbits found in the OC6 catalog. We did not always use the most recent orbits since sometimes older orbits led to equivalent or even smaller residuals.

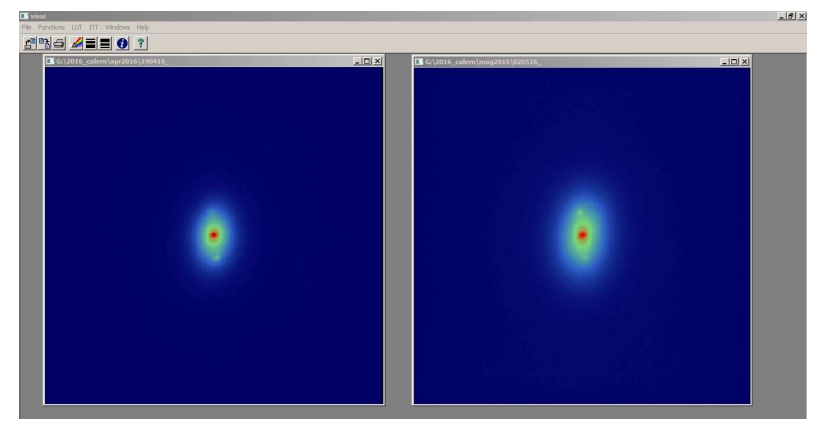

Fig. 7 The Direct Vector Autocorrelation (DVA) images of HU1267 made in April 20th (left) and May 2nd 2016 (right) show an inversion of quadrant which suggests that one of the two components is a variable star.

The $(O-C)$ residuals are plotted in Fig. 8. They have a rather large scatter which is naturally explained by the (old) age of many orbits that will need revision in the future. The mean values computed with the residuals of Table 2 are $\left\langle\Delta \rho_{O-C}\right\rangle=0^{\prime \prime} .007 \pm 0^{\prime \prime} .05$ and $\left\langle\Delta \theta_{O-C}\right\rangle=-0^{\circ} .2 \pm 1^{\circ} .7$. The small values obtained for those offsets provide a good validation of our calibration (see Paper XII).

Let us discuss now the origin of the large residuals found in Fig. 8 for the objects (and the orbits) of A764AB (Hei1995), A1315 (Doc1984b), COU812 (Cou1999), HJ2562 (Pop1996b), and STT260 (Zir2008).

For A764AB (Hei1995), the orbit is old (with observations made before 1994) and it is badly determined (grade 5 according to the grading scale of the OC6). The last observation before our measurement which is indicated in the WDS is from 2016.

For A1315 (Doc1984b), the orbit is very old (with observations made before 1982), with a long period and was not well determined (grade 5) from visual measurements only. The last measurement reported in the WDS was made in 1997 and is also visual.

For HJ2562 (Pop1996b) the orbit is old, has a long period and was not well determined (grade 5). It was only based on visual observations made before 1983 . According to the WDS, the last observation of HJ2562 was made in 1996, but the IC4 only report Hipparcos measurements (in 1991.25).

For COU812 (Cou1999), the orbit is also not well determined (grade 5) and is based on a small arc with a small curvature. It has never been published in a journal or in the Double Stars Information Circular of the International Astronomical Union. The observations indicate a rectilinear trajectory rather than an elliptical orbit.

For STT260 (Zir2008), the orbit is rather recent but the last observation used for this calculation was made in 1997. It is only based on visual measurements and on the Hipparcos measurement. The small grade of 3 


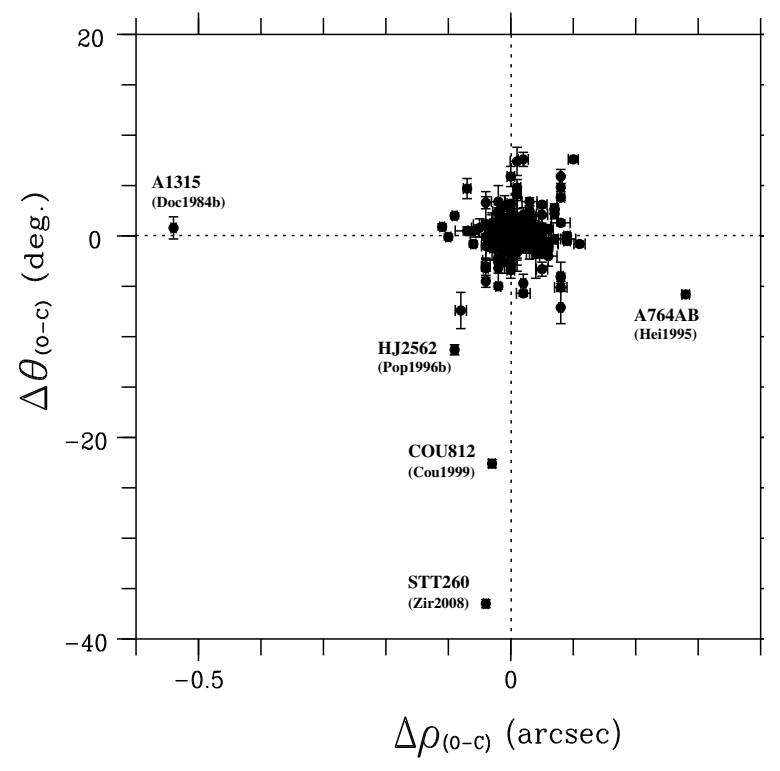

Fig. 8 Residuals of the measurements of Table 2 computed with the published orbits.

that is reported in the OC6 for this orbit seems too optimistic.

\section{Conclusion}

We have presented here the first observations obtained with PISCO with the Epsilon telescope of OCA in the Calern site and an Andor iXon EMCCD camera. The transfer from Merate to Calern included mechanical and optical changes with the development of a new software to control the telescope, the instrument and its camera with real-time processing. This transfer to a more modern environment and better site was successfully completed in less than one year. Compared to the speckle observations made previously with PISCO in Merate, we have been able to increase the overall output efficiency and reach a larger sample of stars with fainter magnitudes.

The data presented here consisted of 1233 observations of 1173 visual binaries or multiple objects made in 2015-2016. The corresponding 1170 new measurements have an average accuracy of $0^{\prime \prime} .0078$ and $0^{\circ} .54$ for the angular separation and position angle, respectively. Our PISCO group has thus succeeded his moving to another telescope and has been pursuing his efforts in providing a good contribution to the continuing monitoring of long period visual binary systems, which is fundamental for refining systemic stellar masses of long period binary systems.

Acknowledgements. We are indebted to the former director of OCA, F. Vakili, who played a key role for moving
PISCO from Merate to OCA. We thank the technical staff of the Brera Astronomical Observatory and of the Côte d'Azur Observatory for their invaluable help for transferring PISCO from Merate to Calern. We are grateful to P. Tanga from Lagrange Laboratory in OCA for allowing us to use his Andor iXon Ultra 897 camera with PISCO. We also thank the members of the U.S. Naval Observatory, Washington DC, for kindly sending us some lists of measurements of visual binaries. This work has made use of the Washington Double Star Catalog (http://ad.usno.navy.mil/wds/wds), the "Fourth Catalog of Interferometric Measurements of Binary Stars" (http://ad.usno.navy.mil/wds/int4), and the "Sixth Catalog of Orbits of Visual Binary Stars" (http://ad.usno.navy.mil/wds/orb6), maintained at the U.S. Naval Observatory. We also used the SIMBAD astronomical data base (http://simbad.u-strasbg.fr/simbad) operated by the Centre de Données Astronomiques de Strasbourg (France).

\section{References}

Aitken, R.G., 1932, "New General Catalog of Double Stars", Carnegie Institute, Washington

Aristidi, E., Carbillet, M., Lyon, J.-F., Aime, C., 1997, A\&AS, 125, 139

Bagnuolo, W.G., Mason, B.D., Barry, D.J., Hartkopf, W.I., McAlister, H.A.: 1992, AJ, 103, 1399

Bendjoya et al., 2012, Proc SF2A, 643

ESA: 1997, The Hipparcos and Tycho Catalogues, ESA SP1200, ESA Publications Division, Noordwijk

Gili, R., Prieur, J.-L., Rivet, J.-P., Vakili, F., Koechlin, L., Bonneau, D., 2014, The Observatory, 134, 267

Hartkopf, W.I., Mason, B.D., 2019, "Sixth Catalog of Orbits of Visual Binary Stars" http://ad.usno.navy.mil/wds/orb6.html (OC6)

Hartkopf, W.I., Mason, B.D., Wycoff, G.L., McAlister, H.A., 2018, "Fourth Catalog of Interferometric Measurements of Binary Stars" http://ad.usno.navy.mil/wds/int4.html (IC4)

Mason, B.D., Wycoff, G.L., Hartkopf, W.I., 2019, "Washington Double Star Catalog" http://ad.usno.navy.mil/wds/wds.html (WDS)

Prieur, J.-L, Koechlin, L., André, C., Gallou, G., Lucuix, C., 1998, Experimental Astronomy, vol 8, Issue 4, 297

Prieur, J.-L., Scardia, M., Pansecchi, L., Argyle, R.W., Sala, M., Ghigo, M., Koechlin, L., Aristidi, E., 2008, MNRAS, 387, 772 (Paper V)

Prieur, J.-L., Scardia, M., Pansecchi, L., Argyle, R.W., Sala, M., 2009, MNRAS, 395, 907 (Paper VII)

Prieur, J.-L., Scardia, M., Pansecchi, L., Argyle, R.W., Sala, M., 2010, MNRAS, 407, 1913 (Paper IX)

Prieur, J.-L., Scardia, M., Pansecchi, L., Argyle, R.W., Sala, M., 2012, MNRAS, 422, 1057 (Paper XI)

Prieur, J.-L., Scardia, M., Pansecchi, L., Argyle, R.W., Zanutta, A., Aristidi, E., 2014, Astron. Nach., 335, 817 (Paper XIII)

Prieur, J.-L., Scardia, M., Pansecchi, L., Argyle, R.W., Zanutta, A., Aristidi, E., 2017, Astron. Nach., 338, 74 (Paper XV)

Scardia, M., Prieur, J.-L., Sala, M., Ghigo, M., Koechlin, L., Aristidi, E., Mazzoleni, F., 2005, MNRAS, 357, 1255 (with erratum in MNRAS 362, 1120) (Paper I) 
Scardia, M., Prieur, J.-L., Pansecchi, L., Argyle, R.W., Sala, M., Ghigo, M., Koechlin, L., Aristidi, E., 2006, MNRAS, 367, 1170 (Paper II)

Scardia, M., Prieur, J.-L., Pansecchi, L., Argyle, R.W., Basso, S., Sala, M., Ghigo, M., Koechlin, L., Aristidi, E., 2007, MNRAS, 374, 965 (Paper III)

Scardia, M., Prieur, J.-L., Pansecchi, L., Argyle, R.W., Sala, M., Basso, S., Ghigo, M., Koechlin, L., Aristidi, E., 2008, Astron. Nach., 329, 1, 54 (Paper IV)

Scardia M., Prieur J.-L., Pansecchi L., Argyle R.W., Sala M., 2009, Astron. Nach., 330, 1, 55 (Paper VI)

Scardia, M., Prieur, J.-L., Pansecchi, L., Argyle, R.W., Sala, M., 2010, Astron. Nach., 331, 286 (Paper VIII)

Scardia, M., Prieur, J.-L., Pansecchi, L., Argyle, R.W., Sala, M., 2011, Astron. Nach., 332, 508 (Paper X)

Scardia, M., Prieur, J.-L., Pansecchi, L., Argyle, R.W., Spanó, P., Riva, M., Landoni, M., 2013, MNRAS, 434, 2803 (Paper XII)

Scardia, M., Prieur, J.L., Pansecchi, L., Argyle, R.W., Zanutta, A., Aristidi, E., 2015, Astron. Nachr., 336, 388 (Paper XIV)

Scardia, M., Prieur, J.L., Pansecchi, L., Argyle, R.W., Zanutta, A., Aristidi, E., 2018, Astron. Nachr., 339, 571 (Paper XVI) 
Table 2 Table of speckle measurements and O-C residuals with published orbits (cont.)

\begin{tabular}{|c|c|c|c|c|c|c|c|c|c|c|c|c|c|}
\hline WDS & Name & ADS & Epoch & Fil. & $\begin{array}{l}\text { Eyep. } \\
(\mathrm{mm})\end{array}$ & $\begin{array}{c}\rho \\
\left({ }^{\prime \prime}\right)\end{array}$ & $\begin{array}{l}\sigma_{\rho} \\
\left({ }^{\prime \prime}\right)\end{array}$ & $\begin{array}{c}\theta \\
\left({ }^{\circ}\right)\end{array}$ & $\begin{array}{l}\sigma_{\theta} \\
\left(^{\circ}\right)\end{array}$ & Notes & Orbit & $\begin{array}{r}\Delta \rho(\mathrm{O}-\mathrm{C}) \\
\left({ }^{\prime \prime}\right)\end{array}$ & $\begin{array}{r}\Delta \theta(\mathrm{O}-\mathrm{C}) \\
\left({ }^{\circ}\right)\end{array}$ \\
\hline $01099+4011$ & AG15 & 953 & 2015.918 & $\mathrm{R}$ & 32 & 2.862 & 0.009 & 69.2 & 0.3 & & & & \\
\hline $01108+5141$ & DOO3 & 968 & 2016.939 & $\mathrm{R}$ & 20 & 0.956 & 0.011 & $354.4^{*}$ & * 0.8 & Elongated & & & \\
\hline $01119+4748$ & BU398 & 978 & 2016.035 & $\mathrm{R}$ & 20 & 1.819 & 0.009 & $43.2^{*}$ & ${ }^{*} 0.3$ & Elongated & & & \\
\hline $01125+3747$ & COU1057 & - & 2016.035 & $\mathrm{R}$ & 20 & 0.428 & 0.004 & $173.0^{*}$ & * 0.7 & & & & \\
\hline $01128+3700$ & COU1058 & - & 2016.035 & $\mathrm{R}$ & 20 & 0.777 & 0.004 & $248.4^{*}$ & * 0.3 & & & & \\
\hline $01128+3700$ & COU1058 & - & 2016.939 & $\mathrm{R}$ & 20 & 0.777 & 0.004 & $249.7^{*}$ & * 0.6 & Elongated & & & \\
\hline $01132+5106$ & HU1024 & 989 & 2016.942 & $\mathrm{R}$ & 20 & 0.742 & 0.007 & $205.9^{*}$ & * 0.4 & & & & \\
\hline $01146+2804$ & A1904 & 1002 & 2016.704 & $\mathrm{R}$ & 20 & 1.210 & 0.006 & 72.1 & 0.3 & & & & \\
\hline $01147+4829$ & COU1858 & - & 2016.942 & $\mathrm{R}$ & 20 & 0.366 & 0.006 & 257.8 & 0.8 & & & & \\
\hline $01151+3416$ & HU803 & 1005 & 2016.704 & $\mathrm{R}$ & 20 & 0.912 & 0.006 & 214.9 & 0.4 & & & & \\
\hline $01161+5554$ & MLR654 & - & 2016.939 & $\mathrm{R}$ & 20 & 0.366 & 0.005 & $210.2^{*}$ & * 0.8 & & & & \\
\hline $01178+4901$ & STF102AB & 1040 & 2016.939 & $\mathrm{R}$ & 20 & 0.472 & 0.007 & $270.8^{*}$ & * 0.8 & & & & \\
\hline $01180+3750$ & COU856 & - & 2016.939 & $\mathrm{R}$ & 20 & 1.166 & 0.006 & $23.6^{*}$ & ${ }^{*} 0.3$ & & & & \\
\hline $01194+0731$ & HEI834 & - & 2016.950 & $\mathrm{R}$ & 20 & 1.057 & 0.008 & $276.7^{*}$ & ${ }^{*} 0.3$ & Elongated & & & \\
\hline $01205+5740$ & MLR561 & - & 2016.950 & $\mathrm{R}$ & 20 & 0.236 & 0.008 & 222.9 & 2.2 & & & & \\
\hline $01207+5136$ & HU523 & 1083 & 2016.950 & $\mathrm{R}$ & 20 & 0.363 & 0.004 & $87.7^{*}$ & * 0.3 & & & & \\
\hline $01208+1813$ & A 2212 & 1094 & 2016.953 & $\mathrm{R}$ & 20 & 1.322 & 0.007 & $213.8^{*}$ & ${ }^{*} 0.3$ & & & & \\
\hline $01226+1449$ & MRL1 & - & 2016.950 & $\mathrm{~V}$ & 10 & 0.180 & 0.003 & 290.6 & 0.7 & Diffuse & & & \\
\hline $01226+1449$ & MRL1 & - & 2016.950 & $\mathrm{R}$ & 10 & 0.189 & 0.007 & 291.3 & 2.2 & Diffuse & & & \\
\hline $01229+0144$ & LV1 & 1110 & 2016.953 & $\mathrm{R}$ & 20 & 0.773 & 0.006 & 327.4 & 0.3 & & & & \\
\hline $01234+5809$ & STF115AB & 1105 & 2016.950 & $\mathrm{R}$ & 20 & 0.428 & 0.006 & $158.5^{*}$ & * 0.5 & & Sod1999 & 0.00 & 1.2 \\
\hline $01258+2733$ & COU666 & - & 2016.953 & $\mathrm{R}$ & 20 & 0.427 & 0.010 & 144.4 & 0.4 & Diffuse & & & \\
\hline $01275+4915$ & HU525 & 1150 & 2016.876 & $\mathrm{R}$ & 20 & 1.159 & 0.006 & $321.3^{*}$ & * 0.3 & & & & \\
\hline $01276+2104$ & COU148 & - & 2016.953 & $\mathrm{R}$ & 20 & 1.211 & 0.006 & $247.0^{*}$ & * 0.4 & Diffuse & & & \\
\hline $01280+5821$ & A940 & 1156 & 2016.732 & $\mathrm{R}$ & 20 & 0.561 & 0.006 & 85.8 & 0.4 & & & & \\
\hline $01283+4247$ & $\mathrm{AC} 14$ & 1161 & 2016.033 & $\mathrm{R}$ & 20 & 0.773 & 0.004 & $91.4^{*}$ & * 0.3 & Elongated & & & \\
\hline $01286+0009$ & RST4748 & - & 2016.876 & $\mathrm{R}$ & 20 & 0.330 & 0.010 & 266.9 & 1.2 & & & & \\
\hline $01292+2004$ & $\mathrm{~A} 2214$ & 1177 & 2016.953 & $\mathrm{R}$ & 20 & 0.724 & 0.004 & $214.2^{*}$ & * 0.6 & & & & \\
\hline $01330+0542$ & J640 & 1213 & 2016.953 & $\mathrm{R}$ & 20 & 1.459 & 0.007 & $49.4^{*}$ & * 0.3 & Diffuse & & & \\
\hline $01352+5150$ & A112 & 1235 & 2016.953 & $\mathrm{R}$ & 20 & 1.121 & 0.006 & $334.7^{*}$ & * 0.3 & & & & \\
\hline $01359+5502$ & MLR629 & - & 2016.953 & $\mathrm{R}$ & 20 & 0.446 & 0.005 & $132.0^{*}$ & * 0.4 & & & & \\
\hline $01365+4336$ & ES1495 & 1255 & 2016.876 & $\mathrm{R}$ & 20 & 1.294 & 0.006 & $66.5^{*}$ & * 0.4 & Elongated & & & \\
\hline $01372+4843$ & A 817 & 1263 & 2016.718 & $\mathrm{R}$ & 20 & 0.457 & 0.004 & 22.8 & 0.9 & & & & \\
\hline $01374+3837$ & TDS1935 & - & 2016.953 & $\mathrm{R}$ & 20 & - & - & - & - & Unres. & & & \\
\hline $01386+5651$ & MLB111AB & 1277 & 2016.876 & $\mathrm{R}$ & 20 & 1.952 & 0.010 & $40.0^{*}$ & * 0.3 & & & & \\
\hline $01391+2656$ & BU508AB & 1294 & 2016.955 & $\mathrm{R}$ & 20 & 0.590 & 0.004 & $52.5^{*}$ & * 1.1 & Elongated & & & \\
\hline $01403+3844$ & BU1167 & 1310 & 2016.956 & $\mathrm{R}$ & 20 & 1.482 & 0.007 & $51.3^{*}$ & * 0.3 & & & & \\
\hline $01406+4447$ & COU1661 & - & 2016.956 & $\mathrm{R}$ & 20 & 1.174 & 0.006 & $242.2^{*}$ & * 0.7 & & & & \\
\hline $01437+0934$ & BU509 & 1360 & 2016.033 & $\mathrm{R}$ & 20 & 0.748 & 0.004 & $42.8^{*}$ & * 0.3 & Elongated & Hrt2010a & -0.03 & -1.2 \\
\hline $01445+3957$ & STF149 & 1368 & 2016.033 & $\mathrm{R}$ & 20 & 1.437 & 0.007 & $80.7^{*}$ & * 0.3 & Elongated & & & \\
\hline $01509+4037$ & COU1361 & - & 2016.939 & W & 20 & 1.125 & 0.011 & $281.9^{*}$ & * 0.3 & & & & \\
\hline $01528-0447$ & RST4188 & - & 2016.936 & $\mathrm{R}$ & 20 & 0.351 & 0.006 & 42.2 & 0.9 & & Tok2015c & -0.00 & 0.3 \\
\hline $01532+1526$ & BU260 & 1503 & 2016.041 & $\mathrm{R}$ & 20 & 1.118 & 0.006 & 259.8 & 0.3 & & Cve2006e & 0.03 & -1.2 \\
\hline $01535+4437$ & STF3113 & 1502 & 2016.041 & $\mathrm{R}$ & 20 & 0.625 & 0.006 & 278.7 & 0.3 & & & & \\
\hline $01548+1728$ & A 2323 & 1520 & 2016.936 & $\mathrm{R}$ & 20 & 1.758 & 0.009 & $147.0^{*}$ & * 0.3 & & & & \\
\hline $01550+5817$ & A954 & 1514 & 2016.041 & $\mathrm{R}$ & 20 & 0.759 & 0.004 & $208.9^{*}$ & * 0.3 & & & & \\
\hline $01563+2520$ & COU 453 & - & 2016.936 & $\mathrm{R}$ & 20 & 0.707 & 0.006 & 282.4 & 0.3 & & & & \\
\hline $01565+5900$ & MLB48 & 1532 & 2016.854 & $\mathrm{R}$ & 20 & 1.277 & 0.006 & $77.4^{*}$ & * 0.5 & & & & \\
\hline $01565+5912$ & MLR486 & - & 2016.936 & $\mathrm{R}$ & 20 & 0.522 & 0.007 & $188.0^{*}$ & * 0.3 & & & & \\
\hline $01573+4620$ & COU1860 & - & 2016.854 & $\mathrm{R}$ & 20 & - & - & - & - & Unres. & & & \\
\hline $02016+4107$ & COU1510 & - & 2016.945 & $\mathrm{R}$ & 20 & 0.379 & 0.007 & $137.4^{*}$ & * 0.5 & & & & \\
\hline $02018+4040$ & A1923 & 1609 & 2016.854 & $\mathrm{R}$ & 20 & 0.664 & 0.010 & $324.2^{*}$ & * 0.3 & & & & \\
\hline $02019+4831$ & COU2009 & - & 2016.857 & $\mathrm{R}$ & 20 & 0.582 & 0.004 & 59.8 & 0.7 & & & & \\
\hline $02020+0246$ & STF202AB & 1615 & 2015.915 & $\mathrm{R}$ & 32 & 1.862 & 0.035 & 262.6 & 0.7 & & Pru2017 & 0.03 & -0.2 \\
\hline $02020+0246$ & STF202AB & 1615 & 2016.879 & $\mathrm{R}$ & 20 & 1.837 & 0.009 & $262.3^{*}$ & * 0.6 & & Pru2017 & 0.00 & 0.1 \\
\hline
\end{tabular}


Table 2 Table of speckle measurements and O-C residuals with published orbits (cont.)

\begin{tabular}{|c|c|c|c|c|c|c|c|c|c|c|c|c|c|}
\hline WDS & Name & $\mathrm{ADS}$ & Epoch & Fil. & $\begin{array}{l}\text { Eyep. } \\
(\mathrm{mm})\end{array}$ & $\underset{\rho}{\rho}$ & $\begin{array}{l}\sigma_{\rho} \\
\left({ }^{\prime \prime}\right)\end{array}$ & $\begin{array}{c}\theta \\
\left({ }^{\circ}\right)\end{array}$ & $\begin{array}{l}\sigma_{\theta} \\
\left(^{\circ}\right)\end{array}$ & Notes & Orbit & $\begin{array}{r}\Delta \rho(\mathrm{O}-\mathrm{C}) \\
\left({ }^{\prime \prime}\right)\end{array}$ & $\begin{array}{r}\Delta \theta(\mathrm{O}-\mathrm{C}) \\
\left({ }^{\circ}\right)\end{array}$ \\
\hline $02021+4311$ & TDS2091 & - & 2016.945 & $\mathrm{R}$ & 20 & - & - & - & - & Unres. & & & \\
\hline $02034+5048$ & TDS2094 & - & 2016.945 & $\mathrm{R}$ & 20 & 0.690 & 0.008 & $333.5^{*}$ & 0.9 & & & & \\
\hline $02055+3018$ & COU455 & - & 2016.879 & $\mathrm{R}$ & 20 & 0.307 & 0.015 & 112.1 & 2.1 & & & & \\
\hline $02062+2207$ & COU149 & - & 2016.879 & $\mathrm{R}$ & 20 & 0.378 & 0.004 & 60.5 & 0.6 & & & & \\
\hline $02064+3854$ & A1926 & 1653 & 2016.854 & $\mathrm{R}$ & 20 & 1.064 & 0.007 & $146.8^{*}$ & 0.3 & & & & \\
\hline $02123+2357$ & STF226AB & 1696 & 2016.098 & $\mathrm{R}$ & 20 & 1.717 & 0.009 & $231.0^{*}$ & 0.3 & & & & \\
\hline $02128+3722$ & HO497 & 1701 & 2016.098 & $\mathrm{R}$ & 20 & 0.466 & 0.004 & $86.8^{*}$ & 0.3 & & & & \\
\hline $02134+5753$ & MLR562 & - & 2016.879 & $\mathrm{R}$ & 20 & 0.714 & 0.004 & $242.5^{*}$ & 0.7 & & & & \\
\hline $02140+4729$ & STF228 & 1709 & 2015.915 & $\mathrm{R}$ & 20 & 0.662 & 0.025 & 300.5 & 0.3 & & Pru2017 & -0.04 & -0.1 \\
\hline $02144+3454$ & HU807 & 1720 & 2016.942 & $\mathrm{R}$ & 20 & 0.500 & 0.005 & $146.0^{*}$ & 0.7 & Elongated & & & \\
\hline $02144+3946$ & A 205 & 1718 & 2016.942 & $\mathrm{R}$ & 20 & 1.741 & 0.009 & $310.4^{*}$ & 0.8 & Elongated & & & \\
\hline $02150+3742$ & COU1368 & - & 2016.942 & $\mathrm{R}$ & 20 & 0.628 & 0.006 & 13.6 & 0.3 & & & & \\
\hline $02150+5903$ & MLR447 & - & 2016.879 & $\mathrm{R}$ & 20 & 0.668 & 0.013 & $342.0^{*}$ & 0.3 & & & & \\
\hline $02162+0919$ & HEI438 & - & 2016.942 & $\mathrm{R}$ & 20 & 0.705 & 0.018 & 160.8 & 0.7 & & & & \\
\hline $02172+5048$ & COU2563 & - & 2016.879 & $\mathrm{R}$ & 20 & 0.873 & 0.009 & 274.3 & 0.3 & & & & \\
\hline $02172+5555$ & STF235 & 1738 & 2016.945 & $\mathrm{R}$ & 20 & 1.896 & 0.009 & $48.2^{*}$ & 0.3 & & & & \\
\hline $02173+5228$ & STF236AB & 1745 & 2016.855 & $\mathrm{R}$ & 20 & 1.449 & 0.007 & $248.6^{*}$ & 0.3 & & & & \\
\hline $02178+5638$ & A1272 & 1748 & 2016.854 & $\mathrm{R}$ & 20 & 1.197 & 0.006 & $23.6^{*}$ & 0.3 & & & & \\
\hline $02193-0259$ & JOY1Aa,Ab & 1778 & 2016.704 & $\mathrm{R}$ & 20 & - & - & - & - & Unres. & & & \\
\hline $02211+2956$ & A962 & 1792 & 2016.098 & $\mathrm{R}$ & 20 & 0.886 & 0.005 & 66.0 & 0.4 & Elongated & & & \\
\hline $02211+4246$ & STF248 & 1786 & 2016.945 & $\mathrm{R}$ & 20 & 0.717 & 0.005 & $205.2^{*}$ & 0.3 & & Pbx2000b & 0.03 & 1.2 \\
\hline $02218+3830$ & STT40 & 1799 & 2015.942 & $\mathrm{R}$ & 20 & 0.675 & 0.015 & $48.1^{*}$ & 0.3 & & & & \\
\hline $02218+3830$ & STT40 & 1799 & 2016.937 & $\mathrm{R}$ & 20 & 0.683 & 0.004 & $48.8^{*}$ & 0.5 & & & & \\
\hline $02218+4349$ & COU1671 & - & 2016.937 & $\mathrm{R}$ & 20 & 0.886 & 0.004 & $255.7^{*}$ & 0.4 & & & & \\
\hline $02223+5737$ & TDS2229 & - & 2016.945 & $\mathrm{R}$ & 20 & 0.600 & 0.005 & 68.9 & 0.7 & & & & \\
\hline $02231+5233$ & HU536 & 1814 & 2016.937 & $\mathrm{R}$ & 20 & 0.634 & 0.011 & $319.9^{*}$ & 0.3 & & & & \\
\hline $02239+5751$ & MLR656 & - & 2016.874 & $\mathrm{R}$ & 20 & 0.638 & 0.004 & $40.7^{*}$ & 0.8 & & & & \\
\hline $02248+5719$ & FOX55 & 1825 & 2016.874 & $\mathrm{R}$ & 20 & 0.835 & 0.005 & $262.5^{*}$ & 0.3 & & & & \\
\hline $02254+5812$ & HLD7 & 1832 & 2016.874 & $\mathrm{R}$ & 20 & 1.702 & 0.009 & $181.8^{*}$ & 0.4 & & & & \\
\hline $02259+3913$ & A1814 & 1842 & 2016.937 & $\mathrm{R}$ & 20 & 0.603 & 0.004 & 33.5 & 0.8 & & & & \\
\hline $02270-0510$ & RST4205 & - & 2016.945 & $\mathrm{R}$ & 20 & 0.762 & 0.012 & $353.0^{*}$ & 0.3 & & & & \\
\hline $02275+5416$ & MLR685 & - & 2016.874 & $\mathrm{R}$ & 20 & 0.388 & 0.008 & $318.3^{*}$ & 1.0 & & & & \\
\hline $02284+1722$ & A 2330 & 1874 & 2016.035 & $\mathrm{R}$ & 20 & 1.192 & 0.006 & $208.4^{*}$ & 0.5 & Elongated & & & \\
\hline $02292+5637$ & A1275 & 1872 & 2016.874 & $\mathrm{R}$ & 20 & 0.843 & 0.004 & $20.9^{*}$ & 0.6 & & & & \\
\hline $02313+4703$ & A968 & 1908 & 2016.016 & $\mathrm{R}$ & 20 & 1.716 & 0.009 & $26.5^{*}$ & 0.3 & & & & \\
\hline $02314+4234$ & A660 & 1913 & 2016.035 & $\mathrm{R}$ & 20 & 0.528 & 0.004 & $310.9^{*}$ & 0.3 & Elongated & & & \\
\hline $02332+4018$ & ES1611 & 1942 & 2016.939 & $\mathrm{R}$ & 20 & 1.524 & 0.008 & $106.7^{*}$ & 0.3 & & & & \\
\hline $02333+5619$ & $\mathrm{~A} 1276 \mathrm{AB}$ & 1934 & 2016.874 & $\mathrm{R}$ & 20 & 0.917 & 0.005 & $201.4^{*}$ & 1.2 & & & & \\
\hline $02348+5246$ & HU203 & 1951 & 2016.874 & $\mathrm{R}$ & 20 & 0.609 & 0.010 & $91.4^{*}$ & 0.5 & & & & \\
\hline $02368-0334$ & BU520 & 1984 & 2016.939 & $\mathrm{R}$ & 20 & 0.872 & 0.007 & $189.8^{*}$ & 0.8 & Elongated & & & \\
\hline $02393+2552$ & A2023 & 2010 & 2016.016 & $\mathrm{R}$ & 20 & 0.513 & 0.004 & 228.8 & 0.5 & & & & \\
\hline $02409+0452$ & STT45 & 2043 & 2016.016 & $\mathrm{R}$ & 20 & 0.795 & 0.004 & $260.5^{*}$ & 0.3 & & & & \\
\hline $02409+0452$ & STT45 & 2043 & 2016.939 & $\mathrm{R}$ & 20 & 0.788 & 0.009 & $259.8^{*}$ & 0.8 & & & & \\
\hline $02446+5643$ & MLR598 & - & 2016.857 & $\mathrm{R}$ & 20 & 0.759 & 0.005 & $79.7^{*}$ & 0.6 & Diffuse & & & \\
\hline $02466+4728$ & TDS2358 & - & 2016.879 & $\mathrm{R}$ & 20 & 0.609 & 0.004 & $52.8^{*}$ & 1.0 & & & & \\
\hline $02471+3533$ & BU9AB & 2117 & 2016.016 & $\mathrm{R}$ & 20 & 0.916 & 0.005 & 215.0 & 0.3 & & Zir2015a & -0.01 & -0.8 \\
\hline $02574+5539$ & A1282 & 2227 & 2016.874 & $\mathrm{R}$ & 20 & 0.641 & 0.005 & $201.6^{*}$ & 1.0 & & & & \\
\hline $03012+4852$ & HU1051 & 2280 & 2016.947 & $\mathrm{R}$ & 20 & 0.512 & 0.006 & 120.8 & 0.6 & Elongated & & & \\
\hline $03014+4021$ & COU1379 & - & 2016.947 & $\mathrm{R}$ & 20 & 0.728 & 0.006 & 76.7 & 0.4 & & & & \\
\hline $03056+5744$ & KR18 & 2327 & 2016.947 & $\mathrm{R}$ & 20 & 1.501 & 0.008 & $273.2^{*}$ & 0.3 & & & & \\
\hline $03058+4342$ & BU1175 & 2334 & 2016.036 & $\mathrm{R}$ & 20 & 0.700 & 0.006 & $272.6^{*}$ & 0.3 & & & & \\
\hline $03069+2052$ & AG61 & 2353 & 2016.036 & $\mathrm{R}$ & 20 & 0.519 & 0.004 & $23.2^{*}$ & 0.3 & Elongated & & & \\
\hline $03076+5230$ & MLR658 & - & 2016.874 & $\mathrm{R}$ & 20 & 0.407 & 0.004 & $87.8^{*}$ & 0.3 & & & & \\
\hline $03085+5639$ & A975 & 2360 & 2016.947 & $\mathrm{R}$ & 20 & 1.611 & 0.009 & 204.6 & 0.3 & & & & \\
\hline
\end{tabular}


Table 2 Table of speckle measurements and O-C residuals with published orbits (cont.)

\begin{tabular}{|c|c|c|c|c|c|c|c|c|c|c|c|c|c|}
\hline WDS & Name & ADS & Epoch & Fil. & $\begin{array}{l}\text { Eyep. } \\
(\mathrm{mm})\end{array}$ & $\begin{array}{c}\rho \\
\left({ }^{\prime \prime}\right)\end{array}$ & $\begin{array}{l}\sigma_{\rho} \\
\left({ }^{\prime \prime}\right)\end{array}$ & $\begin{array}{c}\theta \\
\left(^{\circ}\right)\end{array}$ & $\begin{array}{l}\sigma_{\theta} \\
\left(^{\circ}\right)\end{array}$ & Notes & Orbit & $\begin{array}{r}\Delta \rho(\mathrm{O}-\mathrm{C}) \\
\left({ }^{\prime \prime}\right)\end{array}$ & $\begin{array}{r}\Delta \theta(\mathrm{O}-\mathrm{C}) \\
\left({ }^{\circ}\right)\end{array}$ \\
\hline $03153+5955$ & A977 & 2412 & 2016.874 & $\mathrm{R}$ & 20 & 0.703 & 0.009 & $165.1^{*}$ & * 0.6 & Elongated & & & \\
\hline $03163+1920$ & A2224 & 2439 & 2016.041 & $\mathrm{R}$ & 20 & 0.896 & 0.004 & $331.8^{*}$ & * 0.3 & & & & \\
\hline $03194+5025$ & COU2264 & - & 2016.874 & $\mathrm{R}$ & 20 & 1.270 & 0.013 & $92.3^{*}$ & ${ }^{*} 0.3$ & & & & \\
\hline $03212+2109$ & COU259 & - & 2016.036 & $\mathrm{R}$ & 20 & 0.890 & 0.004 & $216.6^{*}$ & * 0.3 & & & & \\
\hline $03217+0845$ & STF380 & 2491 & 2016.937 & $\mathrm{R}$ & 20 & 0.946 & 0.006 & $5.4^{*}$ & $* 0.4$ & & Pop1996b & 0.07 & 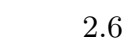 \\
\hline $03248+4159$ & A1288 & 2516 & 2016.033 & $\mathrm{R}$ & 20 & 0.684 & 0.004 & $3.5^{*}$ & ${ }^{*} 0.4$ & Elongated & & & \\
\hline $03252+3837$ & COU1383 & - & 2016.937 & $\mathrm{R}$ & 20 & 0.762 & 0.005 & $284.0^{*}$ & $* 0.3$ & & & & \\
\hline $03261+2015$ & $\mathrm{~A} 2344 \mathrm{AB}$ & 2529 & 2016.033 & $\mathrm{R}$ & 20 & 1.244 & 0.006 & $193.7^{*}$ & * 0.3 & Elongated & & & \\
\hline $03279+4551$ & COU1687 & - & 2016.937 & $\mathrm{R}$ & 20 & 0.795 & 0.004 & $112.0^{*}$ & $* 0.3$ & & & & \\
\hline $03282+5628$ & ES1777 & 2539 & 2016.876 & $\mathrm{R}$ & 20 & 2.317 & 0.012 & $249.7^{*}$ & * 0.3 & & & & \\
\hline $03291+5732$ & MLR663 & - & 2016.937 & $\mathrm{R}$ & 20 & 0.624 & 0.007 & $21.4^{*}$ & * 0.6 & & & & \\
\hline $03294+4931$ & BU1179 & 2558 & 2016.041 & $\mathrm{R}$ & 20 & 0.563 & 0.004 & $141.4^{*}$ & * 0.7 & & & & \\
\hline $03306+4947$ & HLD8 & 2574 & 2016.041 & $\mathrm{R}$ & 20 & 2.363 & 0.012 & $175.9^{*}$ & * 0.3 & & & & \\
\hline $03310+2937$ & A983 & 2578 & 2016.877 & $\mathrm{R}$ & 20 & 0.548 & 0.008 & $144.5^{*}$ & * 0.4 & & Doc2010d & 0.02 & 0.7 \\
\hline $03314+5340$ & MLR664 & - & 2016.953 & $\mathrm{R}$ & 20 & 0.664 & 0.006 & $200.4^{*}$ & * 0.5 & & & & \\
\hline $03318+0749$ & A1931 & 2588 & 2016.041 & $\mathrm{R}$ & 20 & 0.842 & 0.004 & $47.6^{*}$ & * 0.3 & & & & \\
\hline $03318+0749$ & A1931 & 2588 & 2016.956 & $\mathrm{R}$ & 20 & 0.849 & 0.004 & $47.4^{*}$ & * 0.5 & & & & \\
\hline $03331+5159$ & HU101 & 2590 & 2016.877 & $\mathrm{R}$ & 20 & 0.313 & 0.006 & $42.5^{*}$ & * 1.7 & & & & \\
\hline $03333+3643$ & COU1224 & - & 2016.855 & $\mathrm{R}$ & 20 & 0.388 & 0.004 & 31.9 & 1.3 & & & & \\
\hline $03344+2428$ & STF412AB & 2616 & 2015.915 & $\mathrm{R}$ & 20 & 0.743 & 0.025 & 351.4 & 0.3 & & Sca2002a & -0.01 & -0.0 \\
\hline $03344+2428$ & STF412AB & 2616 & 2015.943 & $\mathrm{R}$ & 20 & 0.761 & 0.015 & 351.0 & 0.3 & Elongated & Sca2002a & 0.01 & -0.4 \\
\hline $03344+2428$ & STF412AB & 2616 & 2016.855 & $\mathrm{R}$ & 20 & 0.774 & 0.006 & $352.1^{*}$ & * 0.3 & & Sca2002a & 0.02 & 0.6 \\
\hline $03346+2350$ & COU466AB & - & 2016.956 & $\mathrm{R}$ & 20 & 0.396 & 0.005 & $20.6^{*}$ & * 0.3 & & & & \\
\hline $03354+3529$ & POP83 & - & 2016.956 & $\mathrm{R}$ & 20 & 0.761 & 0.004 & $87.6^{*}$ & * 0.3 & Elongated & & & \\
\hline $03370+1632$ & HEI29 & - & 2016.953 & $\mathrm{R}$ & 20 & 0.794 & 0.004 & $77.7^{*}$ & * 0.4 & & & & \\
\hline $03374+4538$ & TDS2605 & - & 2016.953 & $\mathrm{R}$ & 20 & - & - & - & - & Unres. & & & \\
\hline $03375+4321$ & A1536 & 2641 & 2016.855 & $\mathrm{R}$ & 20 & 1.283 & 0.006 & $227.9^{*}$ & * 0.3 & & & & \\
\hline $03389+4243$ & A1538 & 2652 & 2016.877 & $\mathrm{R}$ & 20 & 0.572 & 0.006 & $263.0^{*}$ & * 0.6 & & & & \\
\hline $03404+1144$ & HEI316 & - & 2016.942 & $\mathrm{R}$ & 20 & 0.868 & 0.014 & 277.6 & 0.3 & & & & \\
\hline $03412+1936$ & A2421 & 2684 & 2016.942 & $\mathrm{R}$ & 20 & 1.186 & 0.011 & 153.0 & 0.3 & Elongated & & & \\
\hline $03425+2946$ & A987 & 2703 & 2016.942 & $\mathrm{R}$ & 20 & 1.142 & 0.013 & 189.9 & 0.3 & & & & \\
\hline $03431+0158$ & RST5201AB & - & 2016.942 & $\mathrm{R}$ & 20 & 0.506 & 0.014 & 339.6 & 1.1 & & & & \\
\hline $03440+5228$ & A1289 & 2710 & 2016.950 & V & 20 & 0.280 & 0.004 & 84.6 & 0.9 & & USN2002 & 0.00 & 0.1 \\
\hline $03463+2411$ & BU536AB & 2755 & 2016.033 & $\mathrm{R}$ & 20 & 1.057 & 0.006 & $177.7^{*}$ & * 0.3 & & Hrt2009 & 0.04 & -1.5 \\
\hline $03473+4011$ & COU1388 & - & 2016.953 & $\mathrm{R}$ & 20 & 0.522 & 0.008 & 300.1 & 0.5 & & & & \\
\hline $03484+5202$ & HU546 & 2768 & 2016.950 & $\mathrm{R}$ & 10 & 0.331 & 0.004 & 359.6 & 0.5 & & Drt2009 & -0.00 & -2.8 \\
\hline $03492+5023$ & HU209AB & 2777 & 2016.937 & $\mathrm{R}$ & 20 & 1.402 & 0.008 & $106.5^{*}$ & * 0.3 & & & & \\
\hline $03503+2241$ & STF457AB & 2801 & 2016.033 & $\mathrm{R}$ & 20 & 0.807 & 0.004 & 90.3 & 0.3 & & & & \\
\hline $03537+5759$ & DRS27AB & - & 2016.950 & $\mathrm{R}$ & 20 & 0.942 & 0.007 & 69.0 & 0.6 & & & & \\
\hline $03554+5630$ & STF461 & 2845 & 2016.950 & $\mathrm{R}$ & 20 & 0.993 & 0.008 & $121.0^{*}$ & * 0.5 & & & & \\
\hline $03556+5216$ & BU743 & 2852 & 2016.950 & $\mathrm{R}$ & 20 & 1.082 & 0.012 & $260.8^{*}$ & * 0.3 & & & & \\
\hline $03570+5427$ & MLR659 & - & 2016.953 & $\mathrm{R}$ & 20 & 0.619 & 0.013 & 157.0 & 0.5 & & & & \\
\hline $03573+5051$ & COU2357 & - & 2016.953 & $\mathrm{R}$ & 20 & 0.839 & 0.009 & $97.5^{*}$ & * 0.6 & & & & \\
\hline $03579+5142$ & SCA176BC & - & 2016.947 & $\mathrm{R}$ & 32 & 8.185 & 0.009 & $76.8^{*}$ & * 0.3 & New pair & & & \\
\hline $03579+5142$ & SCA176BC & - & 2016.950 & $\mathrm{R}$ & 32 & 8.178 & 0.009 & $76.8^{*}$ & * 0.3 & New pair & & & \\
\hline $03579+5142$ & $\mathrm{COU} 2358 \mathrm{Aa}, \mathrm{Ab}$ & - & 2016.947 & $\mathrm{R}$ & 20 & 0.360 & 0.005 & 76.5 & 0.3 & & & & \\
\hline $03584+5645$ & TDS2700 & - & 2016.953 & $\mathrm{R}$ & 20 & 0.668 & 0.004 & $261.5^{*}$ & * 0.4 & & & & \\
\hline $03590+0947$ & HU27 & 2911 & 2016.945 & $\mathrm{R}$ & 20 & 0.450 & 0.010 & $337.6^{*}$ & * 0.4 & & Lin2004a & 0.07 & -0.3 \\
\hline $03594+4321$ & A1708 & 2907 & 2016.945 & $\mathrm{R}$ & 20 & 0.813 & 0.004 & $339.6^{*}$ & * 0.4 & & & & \\
\hline $03597+1128$ & HU28 & 2918 & 2016.945 & $\mathrm{R}$ & 20 & 1.217 & 0.007 & $2.0^{*}$ & * 0.3 & & & & \\
\hline $03598+5013$ & J893 & 2906 & 2016.945 & $\mathrm{R}$ & 20 & 1.079 & 0.005 & $231.3^{*}$ & * 0.3 & Elongated & & & \\
\hline $04016+5044$ & COU2458 & - & 2016.953 & $\mathrm{R}$ & 20 & 0.615 & 0.011 & 145.9 & 0.3 & & & & \\
\hline
\end{tabular}


Table 2 Table of speckle measurements and O-C residuals with published orbits (cont.)

\begin{tabular}{|c|c|c|c|c|c|c|c|c|c|c|c|c|c|}
\hline WDS & Name & ADS & Epoch & Fil. & $\begin{array}{l}\text { Eyep. } \\
(\mathrm{mm})\end{array}$ & $\begin{array}{c}\rho \\
\left({ }^{\prime \prime}\right)\end{array}$ & $\begin{array}{l}\sigma_{\rho} \\
\left({ }^{\prime \prime}\right)\end{array}$ & $\begin{array}{c}\theta \\
\left({ }^{\circ}\right)\end{array}$ & $\begin{array}{l}\sigma_{\theta} \\
\left(^{\circ}\right)\end{array}$ & Notes & Orbit & $\begin{array}{r}\Delta \rho(\mathrm{O}-\mathrm{C}) \\
\left({ }^{\prime \prime}\right)\end{array}$ & $\begin{array}{r}\Delta \theta(\mathrm{O}-\mathrm{C}) \\
\left({ }^{\circ}\right)\end{array}$ \\
\hline $04017+5611$ & HDS507 & - & 2016.137 & $\mathrm{R}$ & 20 & 0.530 & 0.005 & 131.4 & 0.6 & & & & \\
\hline $04081+4535$ & COU2025 & - & 2016.953 & $\mathrm{R}$ & 10 & 0.293 & 0.002 & $344.3^{*}$ & 0.3 & & & & \\
\hline $04081+4535$ & COU2025 & - & 2016.953 & $\mathrm{~V}$ & 10 & 0.297 & 0.002 & 344.0 & 0.5 & & & & \\
\hline $04087+5016$ & HU211 & 3002 & 2016.874 & $\mathrm{R}$ & 20 & 1.600 & 0.010 & $273.6^{*}$ & 0.3 & Elongated & & & \\
\hline $04091+2839$ & HO326 & 3021 & 2016.096 & $\mathrm{R}$ & 20 & 0.397 & 0.006 & 294.6 & 0.8 & & Hrt2008 & 0.00 & -3.4 \\
\hline $04103+5310$ & A1297 & 3025 & 2016.874 & $\mathrm{R}$ & 20 & 0.522 & 0.004 & $348.5^{*}$ & 0.3 & & & & \\
\hline $04115+4152$ & BU546 & 3038 & 2016.099 & $\mathrm{R}$ & 20 & 0.937 & 0.005 & 227.9 & 0.3 & & & & \\
\hline $04123+0939$ & STT74 & 3053 & 2016.099 & $\mathrm{R}$ & 20 & 0.442 & 0.008 & $290.6^{*}$ & 0.3 & Elongated & Alz2003a & 0.10 & $7.6^{Q}$ \\
\hline $04139+0916$ & BU547AB & 3072 & 2016.099 & $\mathrm{R}$ & 20 & 1.264 & 0.006 & $338.9^{*}$ & 0.3 & Elongated & Zir2015a & 0.00 & -0.6 \\
\hline $04140+4235$ & A1711 & 3062 & 2016.033 & $\mathrm{R}$ & 20 & 0.682 & 0.004 & $74.3^{*}$ & 0.4 & & & & \\
\hline $04170+1941$ & HO328 & 3102 & 2016.096 & $\mathrm{R}$ & 20 & 0.441 & 0.004 & 357.5 & 0.5 & & Hei1978a & 0.03 & 0.5 \\
\hline $04199+1631$ & STT79 & 3135 & 2016.096 & $\mathrm{R}$ & 20 & 0.550 & 0.004 & 184.7 & 0.5 & & Alz1999 & 0.00 & -0.1 \\
\hline $04233+1123$ & STF535 & 3174 & 2016.137 & $\mathrm{R}$ & 20 & 1.105 & 0.006 & $268.9^{*}$ & 0.5 & Elongated & Hrt2000c & 0.07 & 2.2 \\
\hline $04243-0452$ & RST4239 & - & 2016.945 & $\mathrm{R}$ & 20 & 0.810 & 0.008 & $107.9^{*}$ & 0.7 & & & & \\
\hline $04247+1346$ & HEI217 & - & 2016.945 & $\mathrm{R}$ & 20 & 0.489 & 0.014 & 150.2 & 1.0 & & & & \\
\hline $04253+1436$ & HEI36 & - & 2016.945 & $\mathrm{R}$ & 20 & 0.782 & 0.008 & $355.2^{*}$ & 0.5 & & & & \\
\hline $04254+5623$ & $\mathrm{~A} 834 \mathrm{AB}$ & 3184 & 2016.036 & $\mathrm{R}$ & 20 & 0.720 & 0.004 & $219.3^{*}$ & 0.3 & & & & \\
\hline $04287+5355$ & BU745 & 3227 & 2016.877 & $\mathrm{R}$ & 20 & 0.383 & 0.008 & 103.3 & 0.9 & & & & \\
\hline $04289+4103$ & A1712 & 3242 & 2016.877 & $\mathrm{R}$ & 20 & 0.399 & 0.004 & $36.3^{*}$ & 1.4 & & & & \\
\hline $04294+4407$ & A1713 & 3246 & 2016.877 & $\mathrm{R}$ & 20 & 0.442 & 0.005 & $200.2^{*}$ & 0.5 & & & & \\
\hline $04324+3849$ & A1839 & 3283 & 2016.033 & $\mathrm{R}$ & 20 & 0.770 & 0.004 & $272.2^{*}$ & 0.3 & & & & \\
\hline $04324+3849$ & A1839 & 3283 & 2016.877 & $\mathrm{R}$ & 20 & 0.785 & 0.004 & $273.1^{*}$ & 0.3 & & & & \\
\hline $04335+1801$ & STF559 & 3297 & 2015.913 & W & 32 & 3.130 & 0.035 & 276.4 & 0.3 & & & & \\
\hline $04335+1801$ & STF559 & 3297 & 2015.913 & $\mathrm{R}$ & 32 & 3.125 & 0.035 & 276.2 & 0.3 & & & & \\
\hline $04347+1130$ & A2034 & 3314 & 2016.016 & $\mathrm{R}$ & 20 & 0.531 & 0.004 & 239.5 & 1.3 & & & & \\
\hline $04349+3908$ & HU1082 & 3303 & 2016.877 & $\mathrm{R}$ & 20 & 0.244 & 0.006 & $189.0^{*}$ & 2.7 & & Cou1975c & 0.04 & -1.5 \\
\hline $04353+4141$ & A1716 & 3310 & 2016.041 & $\mathrm{R}$ & 20 & 0.629 & 0.006 & 89.3 & 0.3 & Faint & & & \\
\hline $04353+4141$ & A1716 & 3310 & 2016.877 & $\mathrm{R}$ & 20 & 0.642 & 0.004 & $90.1^{*}$ & 0.4 & & & & \\
\hline $04366+1946$ & STT86 & 3329 & 2016.096 & $\mathrm{R}$ & 20 & 0.492 & 0.004 & 356.0 & 0.3 & & Zir2010 & 0.02 & -0.8 \\
\hline $04378+4442$ & A1010 & 3332 & 2016.041 & $\mathrm{R}$ & 20 & 0.510 & 0.004 & 343.9 & 0.5 & & & & \\
\hline $04400+5328$ & STF566AB,C & 3358 & 2016.016 & $\mathrm{R}$ & 20 & 0.716 & 0.004 & $166.7^{*}$ & 0.3 & & Cve2008a & -0.10 & -0.1 \\
\hline $04433+5931$ & A1013 & 3391 & 2015.913 & $\mathrm{R}$ & 20 & 0.392 & 0.025 & 296.9 & 0.3 & & Doc1990c & -0.02 & -2.4 \\
\hline $04573-0545$ & RST4260 & - & 2016.939 & $\mathrm{R}$ & 20 & 1.594 & 0.011 & 147.4 & 0.3 & Elongated & & & \\
\hline $04573+1524$ & HEI38 & - & 2016.939 & $\mathrm{R}$ & 20 & 0.628 & 0.004 & 226.6 & 0.9 & Elongated & & & \\
\hline $04574+4204$ & COU1716 & - & 2016.041 & $\mathrm{R}$ & 20 & 0.700 & 0.004 & 151.2 & 0.3 & & & & \\
\hline $05010+1430$ & D6 & 3600 & 2016.033 & $\mathrm{R}$ & 20 & 1.139 & 0.006 & $101.0^{*}$ & 0.3 & & & & \\
\hline $05013+4717$ & COU2461 & - & 2016.937 & $\mathrm{R}$ & 20 & 1.501 & 0.008 & $7.2^{*}$ & 0.3 & & & & \\
\hline $05018+4112$ & A 1552 & 3607 & 2016.939 & $\mathrm{R}$ & 20 & 0.594 & 0.004 & $221.9^{*}$ & 0.7 & & & & \\
\hline $05055+1948$ & STT95 & 3672 & 2016.036 & $\mathrm{R}$ & 20 & 0.938 & 0.005 & $295.1^{*}$ & 0.3 & & Jas1996b & -0.02 & -1.2 \\
\hline $05055+4655$ & A1023 & 3659 & 2015.913 & $\mathrm{R}$ & 20 & 0.227 & 0.035 & 57.3 & 3.6 & & & & \\
\hline $05065+4347$ & COU2465 & - & 2016.937 & $\mathrm{R}$ & 20 & 0.469 & 0.004 & $227.3^{*}$ & 0.8 & & & & \\
\hline $05069+4415$ & COU2466 & - & 2016.939 & $\mathrm{R}$ & 20 & 0.902 & 0.008 & 136.7 & 0.3 & & & & \\
\hline $05071+5920$ & A 1025 & 3673 & 2016.937 & $\mathrm{R}$ & 20 & - & - & - & - & Unres. & & & \\
\hline $05073+2244$ & HU446 & 3700 & 2016.939 & $\mathrm{R}$ & 20 & 0.867 & 0.006 & $189.7^{*}$ & 0.4 & & & & \\
\hline $05079+5459$ & STF635 & 3689 & 2016.137 & $\mathrm{R}$ & 20 & 1.024 & 0.005 & 304.6 & 0.3 & & & & \\
\hline $05092+1629$ & HEI40 & - & 2016.939 & $\mathrm{R}$ & 20 & 0.926 & 0.005 & 179.5 & 0.4 & & & & \\
\hline $05105+0137$ & RST5212 & - & 2016.939 & $\mathrm{R}$ & 20 & 0.714 & 0.005 & 187.9 & 0.7 & Elongated & & & \\
\hline $05115+3938$ & COU1868 & - & 2016.956 & $\mathrm{R}$ & 20 & 0.509 & 0.004 & $189.6^{*}$ & 0.7 & & & & \\
\hline $05118+0102$ & STF652 & 3764 & 2016.036 & $\mathrm{R}$ & 20 & 1.666 & 0.009 & 179.5 & 0.3 & Elongated & & & \\
\hline $05130+0828$ & A 2701 & 3785 & 2016.096 & $\mathrm{R}$ & 20 & 0.500 & 0.004 & 13.4 & 0.3 & & & & \\
\hline $05138+3323$ & HU1223 & 3791 & 2016.942 & $\mathrm{R}$ & 20 & 0.563 & 0.014 & 198.5 & 0.7 & & & & \\
\hline $05140+5126$ & HU821 & 3780 & 2016.942 & $\mathrm{R}$ & 20 & 0.817 & 0.013 & $173.1^{*}$ & 0.4 & & Doc2016c & 0.05 & 0.6 \\
\hline $05153+4710$ & A1031 & 3812 & 2016.033 & $\mathrm{R}$ & 20 & 0.385 & 0.006 & $106.0^{*}$ & 1.0 & & Zir2008 & -0.07 & 4.7 \\
\hline
\end{tabular}


Table 2 Table of speckle measurements and O-C residuals with published orbits (cont.)

\begin{tabular}{|c|c|c|c|c|c|c|c|c|c|c|c|c|c|}
\hline WDS & Name & ADS & Epoch & Fil. & $\begin{array}{l}\text { Eyep. } \\
(\mathrm{mm})\end{array}$ & $\begin{array}{c}\rho \\
\left({ }^{\prime \prime}\right)\end{array}$ & $\begin{array}{l}\sigma_{\rho} \\
\left(^{\prime \prime}\right)\end{array}$ & $\begin{array}{c}\theta \\
\left({ }^{\circ}\right)\end{array}$ & $\begin{array}{l}\sigma_{\theta} \\
\left(^{\circ}\right)\end{array}$ & Notes & Orbit & $\begin{array}{r}\Delta \rho(\mathrm{O}-\mathrm{C}) \\
\left({ }^{\prime \prime}\right)\end{array}$ & $\begin{array}{r}\Delta \theta(\mathrm{O}-\mathrm{C}) \\
\left(^{\circ}\right)\end{array}$ \\
\hline $05163+4447$ & COU2468 & - & 2016.942 & $\mathrm{R}$ & 20 & 0.661 & 0.013 & $327.8^{*}$ & * 1.1 & & & & \\
\hline $05172+3928$ & HU1101 & 3851 & 2016.956 & $\mathrm{R}$ & 20 & 0.418 & 0.004 & $301.1^{*}$ & $* 0.3$ & & & & \\
\hline $05173+3302$ & COU1089 & - & 2016.956 & $\mathrm{R}$ & 20 & 0.932 & 0.005 & $258.2^{*}$ & $* 0.7$ & & & & \\
\hline $05185+0052$ & RST5214 & - & 2016.948 & $\mathrm{R}$ & 20 & 0.951 & 0.007 & 144.7 & 0.4 & & & & \\
\hline $05188+5250$ & STF657 & 3870 & 2016.017 & $\mathrm{R}$ & 20 & 0.955 & 0.005 & $310.3^{*}$ & * 0.3 & & & & \\
\hline $05196+4420$ & COU2469 & - & 2016.948 & $\mathrm{R}$ & 20 & 1.046 & 0.005 & $257.9^{*}$ & $* 0.3$ & & & & \\
\hline $05222+4505$ & STF684 & 3932 & 2016.017 & $\mathrm{R}$ & 20 & 1.500 & 0.007 & $140.9^{*}$ & * 0.3 & & & & \\
\hline $05240+2458$ & STF694AB & 3981 & 2016.017 & $\mathrm{R}$ & 20 & 1.370 & 0.008 & 13.6 & 0.3 & & & & \\
\hline $05243+3939$ & BU1317 & 3976 & 2016.017 & $\mathrm{R}$ & 20 & 0.699 & 0.004 & 15.2 & 0.4 & & & & \\
\hline $05269+3017$ & A1033AB & 4029 & 2016.945 & $\mathrm{R}$ & 20 & 0.391 & 0.007 & $146.6^{*}$ & $* 0.3$ & & & & \\
\hline $05270+2737$ & HO226AB & 4032 & 2016.016 & $\mathrm{R}$ & 20 & 0.664 & 0.004 & 91.6 & 0.3 & & Zir2014a & 0.01 & $-0.6^{Q}$ \\
\hline $05273+2306$ & TDS3196 & - & 2016.945 & $\mathrm{R}$ & 20 & - & - & - & - & Unres. & & & \\
\hline $05275+1830$ & A2432 & 4049 & 2016.945 & $\mathrm{R}$ & 20 & 1.146 & 0.006 & $293.6^{*}$ & * 0.3 & & & & \\
\hline $05295+4807$ & COU2583 & - & 2016.945 & $\mathrm{R}$ & 20 & 0.982 & 0.008 & $60.2^{*}$ & * 0.6 & & & & \\
\hline 05313-0029 & RST4782 & - & 2016.950 & $\mathrm{R}$ & 20 & 0.751 & 0.006 & $67.3^{*}$ & * 0.3 & & & & \\
\hline $05323+1526$ & TDS3231 & - & 2016.950 & $\mathrm{R}$ & 20 & - & - & - & - & Unres. & & & \\
\hline $05340+2225$ & A2106 & 4154 & 2016.950 & $\mathrm{R}$ & 20 & 1.497 & 0.007 & $302.2^{*}$ & * 0.3 & & & & \\
\hline $05349+5025$ & COU2585 & - & 2016.950 & $\mathrm{R}$ & 20 & 0.670 & 0.012 & 126.1 & 0.5 & & & & \\
\hline $05361+5003$ & COU2586 & - & 2016.937 & $\mathrm{R}$ & 20 & - & - & - & - & Unres. & & & \\
\hline $05371+2655$ & STF749AB & 4208 & 2016.033 & $\mathrm{R}$ & 20 & 1.178 & 0.006 & 319.0 & 0.3 & & Sca2007a & 0.01 & -0.9 \\
\hline $05384+5105$ & HU557 & 4215 & 2016.937 & $\mathrm{R}$ & 20 & 0.483 & 0.005 & $285.1^{*}$ & $* 0.9$ & & & & \\
\hline $05416+1913$ & STF770 & 4268 & 2016.099 & $\mathrm{R}$ & 20 & 1.077 & 0.006 & $329.5^{*}$ & * 0.3 & Elongated & & & \\
\hline $05436+1300$ & $\mathrm{~A} 117 \mathrm{AB}$ & 4304 & 2016.033 & $\mathrm{R}$ & 20 & 0.859 & 0.004 & 247.7 & 0.4 & & & & \\
\hline $05447+3118$ & A1040 & 4317 & 2016.033 & $\mathrm{R}$ & 20 & 0.879 & 0.004 & $84.7^{*}$ & * 0.3 & & & & \\
\hline $05449+2149$ & TDS3322 & - & 2016.937 & $\mathrm{R}$ & 20 & 0.642 & 0.006 & $89.6^{*}$ & $* 0.7$ & & & & \\
\hline $05449+2149$ & TDS3322 & - & 2016.937 & $\mathrm{R}$ & 20 & 0.636 & 0.007 & $89.5^{*}$ & $* 0.5$ & & & & \\
\hline $05450+2255$ & HU38 & 4325 & 2016.096 & $\mathrm{R}$ & 20 & 0.459 & 0.004 & 132.4 & 0.3 & & & & \\
\hline $05459+3558$ & HU1232 & 4335 & 2016.877 & $\mathrm{R}$ & 20 & 0.557 & 0.004 & 86.5 & 0.8 & Elongated & & & \\
\hline $05460+2119$ & STF787AB & 4349 & 2016.033 & $\mathrm{R}$ & 20 & 0.664 & 0.004 & $53.7^{*}$ & * 0.3 & & Zir2014a & -0.02 & -1.0 \\
\hline $05474+2939$ & BU560 & 4371 & 2016.096 & $\mathrm{R}$ & 20 & 1.695 & 0.008 & 123.7 & 0.3 & Elongated & Sca2008c & 0.01 & -0.7 \\
\hline $05475+5938$ & A1309 & 4347 & 2016.877 & $\mathrm{R}$ & 20 & 0.440 & 0.005 & $322.6^{*}$ & $* 0.5$ & & & & \\
\hline $06000+4643$ & A1727 & 4565 & 2016.134 & $\mathrm{R}$ & 20 & 0.577 & 0.004 & 247.3 & 0.5 & Elongated & & & \\
\hline $06003+0942$ & $\mathrm{~J} 407$ & 4585 & 2016.134 & $\mathrm{R}$ & 20 & 2.156 & 0.011 & 198.2 & 0.3 & Elongated & & & \\
\hline $06011+4609$ & COU2473 & - & 2016.953 & $\mathrm{R}$ & 20 & 0.892 & 0.006 & $270.6^{*}$ & $* 0.3$ & & & & \\
\hline $06013+2927$ & A119 & 4593 & 2016.099 & $\mathrm{R}$ & 20 & 0.563 & 0.004 & $200.3^{*}$ & $* 0.5$ & Elongated & & & \\
\hline $06032+5813$ & A1315 & 4604 & 2016.953 & $\mathrm{R}$ & 20 & 0.423 & 0.004 & $90.5^{*}$ & * 1.1 & & Doc1984b & -0.54 & $0.8^{Q}$ \\
\hline $06040+3138$ & $\mathrm{~A} 214 \mathrm{AB}$ & 4638 & 2016.953 & $\mathrm{R}$ & 20 & 0.636 & 0.007 & $284.0^{*}$ & * 0.5 & & & & \\
\hline $06040+5106$ & COU2595 & - & 2016.953 & $\mathrm{R}$ & 20 & 0.475 & 0.011 & $112.9^{*}$ & * 1.6 & & & & \\
\hline $06046+4535$ & A1729 & 4639 & 2016.953 & $\mathrm{R}$ & 20 & 0.750 & 0.004 & $74.4^{*}$ & * 0.6 & & & & \\
\hline $06049+4938$ & HU560 & 4640 & 2016.953 & $\mathrm{R}$ & 20 & 0.858 & 0.006 & $20.8^{*}$ & * 0.6 & & & & \\
\hline $06059+5917$ & MLR565 & - & 2016.953 & $\mathrm{R}$ & 20 & 0.349 & 0.004 & $226.8^{*}$ & * 0.3 & & & & \\
\hline $06102+3136$ & TDS223 & - & 2016.134 & $\mathrm{R}$ & 20 & 1.451 & 0.008 & 168.2 & 0.3 & & & & \\
\hline $06109+5330$ & A1572 & 4754 & 2016.137 & $\mathrm{R}$ & 20 & 0.989 & 0.005 & $99.8^{*}$ & $* 0.3$ & & & & \\
\hline $06113+3040$ & STF861BC & 4779 & 2016.137 & $\mathrm{R}$ & 20 & 1.698 & 0.008 & 315.0 & 0.3 & & & & \\
\hline $06126+4733$ & COU2476 & - & 2016.948 & $\mathrm{R}$ & 20 & 0.683 & 0.009 & 101.1 & 0.3 & Elongated & & & \\
\hline $06152+0631$ & $\mathrm{~A} 2717 \mathrm{AB}$ & 4856 & 2016.044 & $\mathrm{R}$ & 20 & 0.529 & 0.009 & 348.2 & 0.3 & & & & \\
\hline $06189+1347$ & HO230 & 4919 & 2016.044 & $\mathrm{R}$ & 20 & 1.608 & 0.008 & 65.2 & 0.4 & & & & \\
\hline $06211+3619$ & A1954 & 4955 & 2016.874 & $\mathrm{R}$ & 20 & 0.671 & 0.010 & $108.1^{*}$ & * 0.3 & Elongated & & & \\
\hline $06254+3544$ & HU831 & 5032 & 2016.874 & $\mathrm{R}$ & 20 & 0.370 & 0.013 & 295.7 & 1.4 & Elongated & & & \\
\hline $06277+1822$ & COU41 & - & 2016.033 & $\mathrm{R}$ & 20 & 1.223 & 0.006 & 39.3 & 0.3 & Faint & & & \\
\hline $06293+1233$ & A 2726 & 5109 & 2016.096 & $\mathrm{R}$ & 20 & 0.580 & 0.004 & 123.9 & 0.3 & Elongated & & & \\
\hline $06307+4336$ & COU2372 & - & 2016.950 & $\mathrm{R}$ & 20 & 0.799 & 0.004 & 3.0 & 1.0 & & & & \\
\hline
\end{tabular}


Table 2 Table of speckle measurements and O-C residuals with published orbits (cont.)

\begin{tabular}{|c|c|c|c|c|c|c|c|c|c|c|c|c|c|}
\hline WDS & Name & ADS & Epoch & Fil. & $\begin{array}{l}\text { Eyep. } \\
(\mathrm{mm})\end{array}$ & $\begin{array}{c}\rho \\
\left({ }^{\prime \prime}\right)\end{array}$ & $\begin{array}{l}\sigma_{\rho} \\
\left({ }^{\prime \prime}\right)\end{array}$ & $\begin{array}{c}\theta \\
\left({ }^{\circ}\right)\end{array}$ & $\begin{array}{l}\sigma_{\theta} \\
\left(^{\circ}\right)\end{array}$ & Notes & Orbit & $\begin{array}{r}\Delta \rho(\mathrm{O}-\mathrm{C}) \\
\left({ }^{\prime \prime}\right)\end{array}$ & $\begin{array}{r}\Delta \theta(\mathrm{O}-\mathrm{C}) \\
\left({ }^{\circ}\right)\end{array}$ \\
\hline $06315+2707$ & TDS3895 & - & 2016.950 & $\mathrm{R}$ & 20 & 1.269 & 0.006 & $113.0^{*}$ & ${ }^{*} 0.3$ & & & & \\
\hline $06316+3210$ & BU896AB & 5151 & 2016.951 & $\mathrm{R}$ & 20 & 0.871 & 0.011 & $179.1^{*}$ & * 0.3 & & & & \\
\hline $06317+2823$ & BU1021 & 5157 & 2016.041 & $\mathrm{R}$ & 20 & 0.683 & 0.004 & $73.7^{*}$ & ${ }^{*} 0.3$ & & & & \\
\hline $06318+2124$ & TDS254 & - & 2016.951 & $\mathrm{R}$ & 20 & 0.834 & 0.008 & $107.3^{*}$ & ${ }^{*} 0.3$ & Elongated & & & \\
\hline $06363+3800$ & BU194 & 5221 & 2016.940 & $\mathrm{R}$ & 20 & 1.537 & 0.009 & $276.1^{*}$ & * 0.5 & Elongated & & & \\
\hline $06368+4415$ & A1051 & 5224 & 2016.940 & $\mathrm{R}$ & 20 & 0.658 & 0.005 & 219.5 & 0.8 & & & & \\
\hline $06379+0855$ & A 2822 & 5273 & 2016.945 & $\mathrm{R}$ & 20 & 0.745 & 0.004 & $122.1^{*}$ & ${ }^{*} 0.4$ & Elongated & & & \\
\hline $06380-0130$ & RST4818 & - & 2016.945 & $\mathrm{R}$ & 20 & 1.011 & 0.005 & $182.9^{*}$ & * 0.3 & Elongated & & & \\
\hline $06383+4201$ & A 2451 & 5261 & 2016.940 & $\mathrm{R}$ & 20 & 0.616 & 0.009 & $145.8^{*}$ & * 0.6 & & & & \\
\hline $06385+1020$ & HEI702 & - & 2016.940 & $\mathrm{R}$ & 20 & 0.828 & 0.004 & $42.2^{*}$ & * 0.6 & & & & \\
\hline $06392+0939$ & STH1 & 5290 & 2016.041 & $\mathrm{R}$ & 20 & 0.735 & 0.004 & 284.3 & 0.3 & & & & \\
\hline $06474+1812$ & STT156 & 5447 & 2016.033 & $\mathrm{R}$ & 10 & 0.180 & 0.008 & 137.5 & 0.7 & & Pru2017 & 0.02 & 7.6 \\
\hline $06511+5425$ & MLR688 & - & 2016.956 & $\mathrm{R}$ & 20 & 1.101 & 0.006 & $94.1^{*}$ & ${ }^{*} 0.4$ & & & & \\
\hline $06555+3010$ & STF981 & 5570 & 2016.044 & $\mathrm{R}$ & 20 & 0.899 & 0.004 & 118.5 & 0.3 & Faint & Kiy2017 & -0.06 & -0.8 \\
\hline $06557+1209$ & TDS299 & - & 2016.940 & $\mathrm{R}$ & 20 & 0.997 & 0.009 & $328.1^{*}$ & * 0.3 & & & & \\
\hline $07004+3008$ & TDS318 & - & 2016.134 & $\mathrm{R}$ & 20 & 1.246 & 0.006 & 20.0 & 0.6 & Faint & & & \\
\hline $07010+2927$ & COU1242 & - & 2016.099 & $\mathrm{R}$ & 20 & 0.347 & 0.006 & 194.6 & 1.0 & & & & \\
\hline $07028+1305$ & HO342 & 5725 & 2016.033 & $\mathrm{R}$ & 20 & 1.188 & 0.006 & $87.5^{*}$ & * 0.3 & & & & \\
\hline $07030+5403$ & A1575 & 5704 & 2016.099 & $\mathrm{R}$ & 20 & 0.802 & 0.004 & $282.7^{*}$ & ${ }^{*} 0.3$ & & & & \\
\hline $07031+5410$ & STF1001BC & 5706 & 2016.099 & $\mathrm{R}$ & 20 & 1.626 & 0.008 & $4.9^{*}$ & * 0.3 & & & & \\
\hline $07062+4023$ & COU2186 & - & 2016.953 & $\mathrm{R}$ & 20 & 0.535 & 0.008 & $94.1^{*}$ & ${ }^{*} 1.1$ & & & & \\
\hline $07065+3736$ & COU2062 & - & 2016.953 & $\mathrm{R}$ & 20 & 0.316 & 0.004 & 247.9 & 1.3 & & & & \\
\hline $07097+4457$ & COU2484 & - & 2016.953 & $\mathrm{R}$ & 20 & 0.984 & 0.005 & $88.7^{*}$ & * 0.3 & & & & \\
\hline $07128+2713$ & STF1037AB & 5371 & 2016.033 & $\mathrm{R}$ & 20 & 0.916 & 0.005 & $304.8^{*}$ & * 0.3 & & Sca2015b & -0.00 & -0.5 \\
\hline $07162+5618$ & MLR567 & - & 2016.953 & $\mathrm{R}$ & 20 & - & - & - & - & Unres. & & & \\
\hline $07170-0004$ & RST5261 & - & 2016.937 & $\mathrm{R}$ & 20 & 0.538 & 0.007 & 347.6 & 0.6 & & & & \\
\hline $07176+0918$ & STT170AB & 5958 & 2016.266 & $\mathrm{R}$ & 20 & 0.460 & 0.007 & 295.6 & 0.5 & Elongated & Sca2016a & 0.01 & -0.5 \\
\hline $07176+0918$ & STT170AB & 5958 & 2016.268 & $\mathrm{R}$ & 20 & 0.461 & 0.005 & $295.4^{*}$ & ${ }^{*} 0.3$ & & Sca2016a & 0.01 & -0.7 \\
\hline $07181+1035$ & HEI725 & - & 2016.937 & $\mathrm{R}$ & 20 & 1.143 & 0.006 & $349.4^{*}$ & * 0.3 & & & & \\
\hline $07260+5310$ & ES772AB & 6054 & 2016.951 & $\mathrm{R}$ & 20 & 1.099 & 0.005 & $99.5^{*}$ & ${ }^{*} 0.3$ & & & & \\
\hline $07346+3153$ & STF1110AB & 6175 & 2016.036 & $\mathrm{R}$ & 32 & 5.147 & 0.026 & $53.3^{*}$ & ${ }^{*} 0.3$ & & DRs2012 & 0.05 & -0.8 \\
\hline $07359+4302$ & STT174 & 6191 & 2016.041 & $\mathrm{R}$ & 20 & 2.156 & 0.011 & $87.9^{*}$ & ${ }^{*} 0.3$ & & & & \\
\hline $07401+0514$ & STF1126AB & 6263 & 2016.271 & $\mathrm{R}$ & 20 & 0.859 & 0.005 & 175.9 & 0.4 & & Zir2015a & 0.03 & -1.0 \\
\hline $07486+2308$ & WRH15AB & 6378 & 2016.036 & $\mathrm{R}$ & 10 & 0.258 & 0.002 & $27.7^{*}$ & ${ }^{*} 1.2$ & & USN2002 & -0.00 & 0.0 \\
\hline $07556+3630$ & COU2075 & - & 2016.033 & $\mathrm{R}$ & 20 & 0.848 & 0.004 & $142.0^{*}$ & ${ }^{*} 0.3$ & & & & \\
\hline $07573+0108$ & STT185 & 6483 & 2016.290 & $\mathrm{R}$ & 20 & 0.410 & 0.004 & $20.2^{*}$ & ${ }^{*} 0.3$ & & Msn2009 & 0.01 & -0.1 \\
\hline $08005+0955$ & A2954BC & 6511 & 2016.266 & $\mathrm{R}$ & 20 & 3.356 & 0.017 & 233.2 & 0.5 & & & & \\
\hline $08005+0955$ & A2954AB & 6511 & 2016.266 & $\mathrm{R}$ & 20 & 0.642 & 0.012 & 338.3 & 0.4 & & & & \\
\hline $08005+0955$ & A2954AC & 6511 & 2016.266 & $\mathrm{R}$ & 20 & 3.577 & 0.018 & 223.3 & 0.3 & & & & \\
\hline $08006+4200$ & ES1538 & 6501 & 2016.266 & $\mathrm{R}$ & 32 & 2.862 & 0.017 & $221.5^{*}$ & ${ }^{*} 0.7$ & & & & \\
\hline $08015+2355$ & A 2540 & 6518 & 2016.099 & $\mathrm{R}$ & 20 & 1.283 & 0.006 & 168.4 & 0.4 & & & & \\
\hline $08033+2616$ & STT186 & 6538 & 2016.042 & $\mathrm{R}$ & 20 & 0.995 & 0.005 & $73.4^{*}$ & ${ }^{*} 0.3$ & & & & \\
\hline $08122+1739$ & STF1196AB & 6650 & 2016.268 & $\mathrm{R}$ & 20 & 1.138 & 0.006 & $17.8^{*}$ & ${ }^{*} 0.3$ & & WSI2006b & 0.01 & 1.9 \\
\hline $08122+1739$ & STF1196AB & 6650 & 2016.268 & $\mathrm{R}$ & 32 & 1.156 & 0.009 & $17.8^{*}$ & ${ }^{*} 0.3$ & & WSI2006b & 0.03 & 1.9 \\
\hline $08122+1739$ & STF1196AC & 6650 & 2016.268 & $\mathrm{R}$ & 32 & 6.353 & 0.032 & $61.3^{*}$ & ${ }^{*} 0.3$ & & & & \\
\hline $08122+1739$ & STF1196BC & 6650 & 2016.268 & $\mathrm{R}$ & 32 & 5.573 & 0.028 & $69.5^{*}$ & ${ }^{*} 0.3$ & & & & \\
\hline $08285-0231$ & $\mathrm{~A} 551 \mathrm{AB}$ & 6828 & 2016.268 & $\mathrm{R}$ & 20 & 0.336 & 0.005 & $61.1^{*}$ & ${ }^{*} 1.0$ & & Doc2017a & -0.01 & -0.7 \\
\hline $08285-0231$ & $\mathrm{~A} 551 \mathrm{AB}$ & 6828 & 2016.287 & $\mathrm{R}$ & 20 & 0.339 & 0.004 & 61.2 & 1.7 & & Doc2017a & -0.00 & -0.6 \\
\hline $08413+2029$ & BU585 & 6930 & 2016.042 & $\mathrm{R}$ & 20 & 0.291 & 0.005 & $72.1^{*}$ & ${ }^{*} 0.7$ & & & & \\
\hline $08444+1555$ & A2472 & 6963 & 2016.033 & $\mathrm{R}$ & 20 & 0.813 & 0.004 & 261.0 & 0.3 & & & & \\
\hline $08482+0235$ & BU335 & 7003 & 2016.287 & $\mathrm{R}$ & 20 & 2.565 & 0.013 & 265.4 & 0.3 & Faint & & & \\
\hline $08500+3935$ & STF1279 & 7019 & 2016.271 & $\mathrm{R}$ & 20 & 1.251 & 0.006 & 88.5 & 0.3 & & & & \\
\hline $08507+1800$ & A 2473 & 7039 & 2016.287 & $\mathrm{R}$ & 10 & 0.247 & 0.003 & 92.4 & 1.6 & & Doc2016d & 0.08 & -7.1 \\
\hline $08512+0820$ & PER1 & 7046 & 2016.042 & $\mathrm{R}$ & 20 & 0.902 & 0.006 & $351.4^{*}$ & ${ }^{*} 0.3$ & Elongated & & & \\
\hline
\end{tabular}


Table 2 Table of speckle measurements and O-C residuals with published orbits (cont.)

\begin{tabular}{|c|c|c|c|c|c|c|c|c|c|c|c|c|c|}
\hline WDS & Name & ADS & Epoch & Fil. & $\begin{array}{l}\text { Eyep. } \\
(\mathrm{mm})\end{array}$ & $\begin{array}{c}\rho \\
\left({ }^{\prime \prime}\right)\end{array}$ & $\begin{array}{l}\sigma_{\rho} \\
\left({ }^{\prime \prime}\right)\end{array}$ & $\begin{array}{c}\theta \\
\left({ }^{\circ}\right)\end{array}$ & $\begin{array}{l}\sigma_{\theta} \\
\left(^{\circ}\right)\end{array}$ & Notes & Orbit & $\begin{array}{r}\Delta \rho(\mathrm{O}-\mathrm{C}) \\
\left({ }^{\prime \prime}\right)\end{array}$ & $\begin{array}{r}\Delta \theta(\mathrm{O}-\mathrm{C}) \\
\left({ }^{\circ}\right)\end{array}$ \\
\hline $08514+5732$ & STF1275AB & 7053 & 2016.271 & $\mathrm{R}$ & 20 & 1.877 & 0.009 & 198.2 & 0.3 & & & & \\
\hline $08539+1958$ & COU773 & - & 2016.287 & $\mathrm{R}$ & 10 & - & - & - & - & Unres. & & & \\
\hline $08561+4341$ & STF3120AB & 7092 & 2016.271 & $\mathrm{R}$ & 20 & 1.350 & 0.007 & 1.2 & 0.3 & & & & \\
\hline $08571+1045$ & A2968 & 7102 & 2016.290 & $\mathrm{R}$ & 20 & 1.174 & 0.007 & 133.7 & 0.3 & & & & \\
\hline $08580+3014$ & HO252 & 7107 & 2016.268 & $\mathrm{R}$ & 10 & - & - & - & - & Unres. & & & \\
\hline $09006+4147$ & KUI37AB & - & 2016.096 & $\mathrm{R}$ & 20 & 0.479 & 0.004 & $169.8^{*}$ & 0.8 & & Mut2010b & 0.01 & -0.8 \\
\hline $09006+4147$ & KUI37AB & - & 2016.134 & $\mathrm{R}$ & 20 & 0.465 & 0.005 & $169.7^{*}$ & 0.6 & Elongated & Mut2010b & -0.01 & -0.9 \\
\hline $09007+3208$ & COU1253 & - & 2016.134 & $\mathrm{R}$ & 20 & 0.722 & 0.004 & 189.7 & 0.6 & Elongated & & & \\
\hline $09020+0240$ & BU211 & 7152 & 2016.137 & $\mathrm{R}$ & 20 & 1.093 & 0.005 & 269.3 & 0.3 & & & & \\
\hline $09033+4740$ & HU720 & 7153 & 2016.042 & $\mathrm{R}$ & 20 & 0.748 & 0.004 & 133.7 & 0.3 & & & & \\
\hline $09035+3750$ & MUG1 & - & 2016.096 & $\mathrm{RL}$ & 20 & 1.689 & 0.009 & 1.0 & 0.4 & Faint & & & \\
\hline $09067+5038$ & HU722 & 7177 & 2016.293 & $\mathrm{R}$ & 20 & 0.494 & 0.004 & 236.8 & 0.7 & & & & \\
\hline $09088+4416$ & COU2619 & - & 2016.304 & $\mathrm{R}$ & 20 & 1.161 & 0.006 & $240.1^{*}$ & 0.4 & & & & \\
\hline $09095+0256$ & STT197 & 7215 & 2016.134 & $\mathrm{R}$ & 20 & 1.392 & 0.007 & $66.3^{*}$ & 0.4 & Elongated & & & \\
\hline $09096+1606$ & $\mathrm{~A} 2475$ & 7213 & 2016.266 & $\mathrm{R}$ & 20 & 0.379 & 0.011 & 173.8 & 0.5 & & & & \\
\hline $09097+0013$ & $\mathrm{~J} 424$ & 7216 & 2016.268 & $\mathrm{R}$ & 20 & 1.352 & 0.011 & $141.8^{*}$ & 0.3 & Faint & & & \\
\hline $09101+3534$ & TDS6367 & - & 2016.265 & $\mathrm{R}$ & 20 & - & - & - & - & Unres. & & & \\
\hline $09127+1632$ & STF1322 & 7236 & 2016.134 & $\mathrm{R}$ & 20 & 1.777 & 0.009 & 52.9 & 0.3 & Elongated & & & \\
\hline $09136+4659$ & STF1318 & 7243 & 2016.290 & $\mathrm{R}$ & 20 & 2.589 & 0.013 & 228.4 & 0.3 & & & & \\
\hline 09143-0430 & RST4432 & - & 2016.268 & $\mathrm{R}$ & 32 & 1.483 & 0.009 & 172.8 & 0.5 & Faint & & & \\
\hline $09149+0413$ & BU455 & 7257 & 2016.137 & $\mathrm{R}$ & 20 & 1.846 & 0.009 & $68.0^{*}$ & 0.4 & & & & \\
\hline 09168-0050 & RST4906 & - & 2016.342 & $\mathrm{R}$ & 20 & 0.859 & 0.009 & 154.2 & 0.3 & & & & \\
\hline $09184+3522$ & STF1333 & 7286 & 2016.165 & $\mathrm{R}$ & 20 & 1.930 & 0.010 & $50.0^{*}$ & 0.4 & & & & \\
\hline $09186+2049$ & $\mathrm{HO} 43$ & 7294 & 2016.293 & $\mathrm{R}$ & 20 & 0.607 & 0.007 & 97.8 & 0.4 & Faint & Sca2016a & 0.00 & -0.3 \\
\hline $09186+2049$ & $\mathrm{HO} 43$ & 7294 & 2016.296 & $\mathrm{R}$ & 20 & 0.600 & 0.010 & 97.2 & 0.3 & Elongated & Sca2016a & 0.01 & 0.4 \\
\hline $09210+3643$ & STF1339 & - & 2016.042 & $\mathrm{R}$ & 20 & 1.460 & 0.007 & $64.8^{*}$ & 0.3 & & & & \\
\hline $09210+3811$ & STF1338AB & - & 2016.096 & $\mathrm{R}$ & 20 & 1.129 & 0.006 & $309.1^{*}$ & 0.3 & & Sca2002b-II & -0.03 & -0.2 \\
\hline $09215+1943$ & $\mathrm{~A} 127$ & 7321 & 2016.296 & $\mathrm{R}$ & 20 & 1.430 & 0.007 & 32.5 & 0.3 & & & & \\
\hline $09232+1008$ & J386 & 7337 & 2016.296 & $\mathrm{R}$ & 20 & 0.459 & 0.012 & 52.6 & 2.2 & Elongated & & & \\
\hline $09233+2211$ & AG165 & 7336 & 2016.137 & $\mathrm{R}$ & 20 & 1.330 & 0.007 & 14.1 & 0.3 & & & & \\
\hline $09239+2754$ & STT201AB & 7344 & 2016.099 & $\mathrm{R}$ & 20 & 1.243 & 0.006 & $204.2^{*}$ & 0.3 & & & & \\
\hline $09245+1808$ & A2477 & 7341 & 2016.287 & $\mathrm{R}$ & 20 & 0.456 & 0.013 & $4.9^{*}$ & 1.9 & Elongated & Fmr2014a & 0.03 & -0.4 \\
\hline $09247+3641$ & VBS16 & - & 2016.296 & $\mathrm{R}$ & 20 & 1.612 & 0.031 & 282.4 & 0.6 & & & & \\
\hline $09249+5134$ & STT200 & 7348 & 2016.282 & $\mathrm{R}$ & 20 & 1.257 & 0.024 & $334.3^{*}$ & 0.4 & & & & \\
\hline $09252+1449$ & HU869 & 7359 & 2016.099 & $\mathrm{R}$ & 20 & 0.775 & 0.007 & $273.0^{*}$ & 0.3 & & & & \\
\hline $09260+2839$ & $\mathrm{~A} 222$ & 7365 & 2016.342 & $\mathrm{R}$ & 20 & 0.390 & 0.005 & $5.1^{*}$ & 1.0 & Elongated & Hrt2014b & -0.01 & -0.2 \\
\hline $09277+4456$ & A1762 & 7378 & 2016.099 & $\mathrm{R}$ & 20 & 0.789 & 0.004 & 104.6 & 0.3 & & & & \\
\hline $09285+0903$ & STF1356 & 7390 & 2016.282 & $\mathrm{R}$ & 20 & 0.830 & 0.018 & $111.1^{*}$ & 0.8 & & Mut2010b & -0.01 & 0.5 \\
\hline $09290+1917$ & COU936 & - & 2016.282 & $\mathrm{R}$ & 20 & 0.950 & 0.005 & $2225.1^{*}$ & 0.3 & & & & \\
\hline $09300+4216$ & A1985 & 7398 & 2016.288 & $\mathrm{R}$ & 20 & 1.591 & 0.008 & 24.4 & 0.3 & & & & \\
\hline $09435+0612$ & A2761 & 7499 & 2016.301 & $\mathrm{R}$ & 20 & 1.043 & 0.005 & 251.1 & 0.3 & & & & \\
\hline $09478+1004$ & HO253 & 7517 & 2016.329 & $\mathrm{R}$ & 20 & 1.250 & 0.006 & $298.3^{*}$ & 0.3 & & & & \\
\hline 09496-0017 & RST5339 & - & 2016.329 & $\mathrm{R}$ & 20 & 0.805 & 0.004 & 195.8 & 0.6 & & & & \\
\hline $09498+2111$ & KUI44AB & - & 2016.282 & $\mathrm{R}$ & 10 & 0.172 & 0.005 & 227.0 & 3.1 & & Doc2005g & 0.01 & 1.8 \\
\hline $09498+2111$ & KUI44AB & - & 2016.282 & $\mathrm{R}$ & 10 & 0.178 & 0.002 & 225.7 & 4.0 & & Doc2005g & 0.01 & 0.5 \\
\hline $09502+5706$ & MLR568 & - & 2016.329 & $\mathrm{R}$ & 20 & 0.569 & 0.004 & 56.5 & 0.3 & & & & \\
\hline $09521+2916$ & A344 & 7547 & 2016.329 & $\mathrm{R}$ & 20 & 0.647 & 0.005 & $70.4^{*}$ & 0.6 & Elongated & & & \\
\hline $09521+5404$ & STT208 & 7545 & 2016.282 & $\mathrm{R}$ & 10 & 0.408 & 0.009 & 307.0 & 0.3 & & Hei1996c & -0.02 & -0.1 \\
\hline $09566+4359$ & POP151 & - & 2016.326 & $\mathrm{R}$ & 20 & 0.479 & 0.004 & 265.5 & 0.3 & & & & \\
\hline $09571-0121$ & A1766AB & 7592 & 2016.326 & $\mathrm{R}$ & 20 & 0.521 & 0.005 & $189.9^{*}$ & 0.4 & Elongated & & & \\
\hline $09581+3856$ & COU2086 & - & 2016.326 & $\mathrm{R}$ & 10 & - & - & - & - & Unres. & & & \\
\hline $09584+4045$ & HDS1437 & - & 2016.268 & $\mathrm{R}$ & 20 & - & - & - & - & Unres. & & & \\
\hline $09591+5316$ & A1346 & 7598 & 2016.326 & $\mathrm{R}$ & 20 & 0.580 & 0.004 & $178.8^{*}$ & 0.3 & & Hrt2009 & -0.02 & -0.4 \\
\hline $09593+3746$ & COU2088 & - & 2016.268 & $\mathrm{R}$ & 20 & 0.448 & 0.007 & $131.3^{*}$ & 0.7 & & & & \\
\hline
\end{tabular}


Table 2 Table of speckle measurements and O-C residuals with published orbits (cont.)

\begin{tabular}{|c|c|c|c|c|c|c|c|c|c|c|c|c|c|}
\hline WDS & Name & ADS & Epoch & Fil. & $\begin{array}{l}\text { Eyep. } \\
\text { (mm) }\end{array}$ & $\begin{array}{c}\rho \\
\left({ }^{\prime \prime}\right)\end{array}$ & $\begin{array}{l}\sigma_{\rho} \\
\left({ }^{\prime \prime}\right)\end{array}$ & $\begin{array}{c}\theta \\
\left(^{\circ}\right)\end{array}$ & $\begin{array}{l}\sigma_{\theta} \\
\left({ }^{\circ}\right)\end{array}$ & Notes & Orbit & $\begin{array}{r}\Delta \rho(\mathrm{O}-\mathrm{C}) \\
\left({ }^{\prime \prime}\right)\end{array}$ & $\begin{array}{r}\Delta \theta(\mathrm{O}-\mathrm{C}) \\
\left({ }^{\circ}\right)\end{array}$ \\
\hline $10022+5323$ & MLR678 & - & 2016.268 & $\mathrm{R}$ & 20 & - & - & - & - & Unres. & & & \\
\hline $10026+4622$ & STT210 & 7613 & 2016.135 & $\mathrm{R}$ & 20 & 1.276 & 0.006 & $256.6^{*}$ & * 0.3 & & & & \\
\hline $10040+3239$ & HU631 & 7624 & 2016.268 & $\mathrm{R}$ & 20 & 0.805 & 0.005 & 256.3 & 0.4 & & & & \\
\hline $10057+4103$ & A 2142 & 7631 & 2016.135 & $\mathrm{R}$ & 20 & 1.070 & 0.005 & $294.9^{*}$ & * 0.3 & & & & \\
\hline $10083+0802$ & A2564 & 7652 & 2016.266 & $\mathrm{R}$ & 20 & 0.771 & 0.011 & $285.4^{*}$ & * 0.6 & Elongated & & & \\
\hline $10095+4126$ & A2143 & 7660 & 2016.326 & $\mathrm{R}$ & 20 & 0.305 & 0.004 & 159.0 & 0.4 & & & & \\
\hline $10131+2725$ & STT213AB & 7685 & 2016.135 & $\mathrm{R}$ & 20 & 1.130 & 0.006 & $121.3^{*}$ & * 0.3 & & Sca2008e & 0.05 & 0.8 \\
\hline $10140+2227$ & COU169Aa,Ab & - & 2016.326 & $\mathrm{R}$ & 20 & 0.528 & 0.011 & 325.8 & 0.3 & & Cou1999b & 0.02 & -5.7 \\
\hline $10152+1153$ & HU1254 & 7696 & 2016.329 & $\mathrm{R}$ & 20 & 0.585 & 0.004 & 14.8 & 1.0 & Elongated & & & \\
\hline $10163+1744$ & STT215 & 7704 & 2016.271 & $\mathrm{R}$ & 20 & 1.487 & 0.007 & 176.5 & 0.3 & & Sca2018 & -0.01 & -0.1 \\
\hline $10182+0805$ & A2765 & 7718 & 2016.326 & $\mathrm{R}$ & 20 & 0.413 & 0.005 & $34.6^{*}$ & * 1.8 & Elongated & & & \\
\hline $10184+3730$ & HU875 & 7717 & 2016.329 & $\mathrm{R}$ & 20 & 1.177 & 0.006 & $74.5^{*}$ & * 0.3 & & & & \\
\hline $10184+4346$ & POP117 & - & 2016.337 & $\mathrm{R}$ & 20 & 0.763 & 0.004 & $261.8^{*}$ & * 0.3 & & & & \\
\hline $10192+2034$ & STF1423 & 7721 & 2016.337 & $\mathrm{R}$ & 20 & 0.699 & 0.006 & $133.7^{*}$ & * 0.3 & Elongated & WSI2004a & 0.08 & $3.8^{Q}$ \\
\hline $10227+1521$ & STT216 & 7744 & 2016.288 & $\mathrm{R}$ & 20 & 2.283 & 0.011 & 230.8 & 0.3 & & Sca2009c & 0.03 & 0.2 \\
\hline $10234+2630$ & A1990 & 7747 & 2016.288 & $\mathrm{R}$ & 20 & 1.485 & 0.007 & 291.1 & 0.3 & Elongated & & & \\
\hline $10236+2617$ & A1991 & 7748 & 2016.288 & $\mathrm{R}$ & 20 & 1.380 & 0.013 & 189.8 & 0.4 & Elongated & & & \\
\hline $10256+0302$ & HEI761 & - & 2016.329 & $\mathrm{R}$ & 20 & 1.185 & 0.006 & 171.9 & 0.3 & Faint & & & \\
\hline $10261+5347$ & MLR679 & - & 2016.329 & $\mathrm{R}$ & 20 & 1.100 & 0.006 & 83.1 & 0.3 & Faint & & & \\
\hline $10262+0302$ & DJU2 & - & 2016.337 & $\mathrm{R}$ & 20 & - & - & - & - & & & & \\
\hline $10279+3642$ & HU879 & 7780 & 2016.288 & $\mathrm{R}$ & 20 & 0.549 & 0.008 & $226.9^{*}$ & ${ }^{*} 1.3$ & & Msn2001c & 0.00 & 0.5 \\
\hline $10362+0041$ & $\mathrm{~J} 84$ & 7845 & 2016.301 & $\mathrm{R}$ & 20 & 0.435 & 0.005 & 204.4 & 1.1 & & & & \\
\hline $10376+3446$ & COU1417 & - & 2016.301 & $\mathrm{R}$ & 20 & 0.312 & 0.005 & 211.8 & 0.6 & & & & \\
\hline $10382+2636$ & STF1454 & - & 2016.340 & $\mathrm{R}$ & 20 & 1.296 & 0.006 & $178.0^{*}$ & ${ }^{*} 0.3$ & Faint & & & \\
\hline $10382+4558$ & COU2092 & - & 2016.340 & $\mathrm{R}$ & 20 & 0.613 & 0.008 & 281.2 & 0.3 & & & & \\
\hline $10397+0851$ & STT224AB & 7871 & 2016.288 & $\mathrm{R}$ & 20 & 0.492 & 0.006 & $132.8^{*}$ & * 0.6 & & Hrt2010a & 0.00 & -0.4 \\
\hline $10426+0335$ & A2768 & 7896 & 2016.288 & $\mathrm{R}$ & 20 & 0.626 & 0.004 & $242.7^{*}$ & * 1.9 & & Hrt2010a & 0.02 & 0.4 \\
\hline $10474-0053$ & RST4931 & - & 2016.301 & $\mathrm{R}$ & 20 & 0.838 & 0.011 & 309.3 & 0.3 & & & & \\
\hline $10480+4107$ & STT229 & 7929 & 2016.247 & $\mathrm{R}$ & 20 & 0.657 & 0.004 & 258.2 & 0.5 & Elongated & Alz1998a & 0.03 & 2.1 \\
\hline $11000-0328$ & STF 1500 & 8007 & 2016.135 & $\mathrm{R}$ & 20 & 1.322 & 0.007 & $299.4^{*}$ & * 0.3 & Elongated & Hrt2013a & -0.01 & -0.3 \\
\hline $11018+2952$ & COU961AB & - & 2016.269 & $\mathrm{R}$ & 20 & 1.169 & 0.006 & $327.5^{*}$ & * 0.3 & & & & \\
\hline $11023+3049$ & STF1501 & 8023 & 2016.247 & $\mathrm{R}$ & 20 & 1.303 & 0.007 & 185.3 & 0.3 & & & & \\
\hline $11029+3541$ & $\mathrm{HO} 47 \mathrm{BC}$ & 8031 & 2016.337 & $\mathrm{R}$ & 20 & 0.883 & 0.008 & 338.7 & 0.3 & & & & \\
\hline $11035+5432$ & A1590 & 8032 & 2016.282 & $\mathrm{R}$ & 20 & 1.465 & 0.007 & $328.4^{*}$ & * 0.3 & & Baz1985b & -0.04 & -3.0 \\
\hline $11040+0338$ & STF1504 & 8043 & 2016.296 & $\mathrm{R}$ & 20 & 1.211 & 0.017 & 124.5 & 0.3 & & & & \\
\hline $11040+2309$ & $\mathrm{HO} 48$ & 8041 & 2016.269 & $\mathrm{R}$ & 20 & 1.833 & 0.009 & $355.3^{*}$ & ${ }^{*} 0.4$ & & & & \\
\hline $11046+5058$ & COU2505 & - & 2016.326 & $\mathrm{R}$ & 20 & 0.474 & 0.004 & 254.1 & 0.6 & & & & \\
\hline $11050+3825$ & HO378AB & 8047 & 2016.290 & $\mathrm{R}$ & 20 & 1.073 & 0.005 & $237.1^{*}$ & * 0.4 & Elongated & & & \\
\hline $11078+3946$ & COU1749 & - & 2016.296 & $\mathrm{R}$ & 20 & 0.524 & 0.004 & 109.2 & 0.6 & Faint & & & \\
\hline $11080+1012$ & J81 & 8067 & 2016.296 & $\mathrm{R}$ & 20 & 1.901 & 0.039 & $137.8^{*}$ & * 0.3 & & & & \\
\hline $11080+2659$ & TDS7688 & - & 2016.337 & $\mathrm{R}$ & 20 & 0.538 & 0.008 & $14.0^{*}$ & * 1.3 & & & & \\
\hline $11098+1009$ & A 2775 & 8077 & 2016.337 & $\mathrm{R}$ & 20 & 0.569 & 0.009 & $122.2^{*}$ & * 0.5 & & & & \\
\hline $11100+2138$ & COU176 & 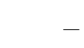 & 2016.326 & $\mathrm{R}$ & 20 & 0.809 & 0.007 & $76.3^{*}$ & * 0.4 & Faint & & & \\
\hline $11107+3110$ & HJ2562 & 8082 & 2016.291 & $\mathrm{R}$ & 20 & 0.977 & 0.005 & $222.8^{*}$ & * 0.5 & Elongated & Pop1996b & -0.09 & -11.3 \\
\hline $11125+3205$ & COU963 & - & 2016.337 & $\mathrm{R}$ & 20 & 1.705 & 0.009 & 191.2 & 0.3 & & & & \\
\hline $11128+1031$ & HEI500 & - & 2016.337 & $\mathrm{R}$ & 20 & 1.544 & 0.008 & 80.5 & 0.4 & Faint & & & \\
\hline $11136+5525$ & A1353 & 8092 & 2016.282 & $\mathrm{R}$ & 20 & 0.566 & 0.004 & 208.6 & 1.0 & & Doc2015f & 0.01 & 0.1 \\
\hline $11151+3735$ & STT232AB & 8102 & 2016.247 & $\mathrm{R}$ & 20 & 0.622 & 0.004 & 245.2 & 0.3 & & & & \\
\hline $11158+4227$ & COU1904 & - & 2016.291 & $\mathrm{R}$ & 20 & 0.379 & 0.004 & 213.6 & 1.1 & & & & \\
\hline $11190+1416$ & STF1527 & 8128 & 2016.266 & $\mathrm{R}$ & 10 & 0.324 & 0.003 & $265.7^{*}$ & * 0.6 & & Tok2012b & 0.00 & 1.3 \\
\hline $11190+1416$ & STF 1527 & 8128 & 2016.288 & $\mathrm{R}$ & 10 & 0.314 & 0.004 & 266.0 & 2.2 & & Tok2012b & 0.01 & 1.3 \\
\hline $11203+3603$ & COU1259 & - & 2016.266 & $\mathrm{R}$ & 20 & 0.742 & 0.005 & $310.5^{*}$ & ${ }^{*} 0.4$ & & & & \\
\hline $11221+3705$ & COU1260BC & - & 2016.342 & $\mathrm{R}$ & 20 & 0.404 & 0.008 & $13.3^{*}$ & ${ }^{*} 0.5$ & & & & \\
\hline $11237-0442$ & RST4475 & - & 2016.386 & $\mathrm{R}$ & 20 & 0.619 & 0.006 & 131.3 & 0.3 & Elongated & & & \\
\hline
\end{tabular}


Table 2 Table of speckle measurements and O-C residuals with published orbits (cont.)

\begin{tabular}{|c|c|c|c|c|c|c|c|c|c|c|c|c|c|}
\hline WDS & Name & ADS & Epoch & Fil. & $\begin{array}{l}\text { Eyep. } \\
(\mathrm{mm})\end{array}$ & $\begin{array}{c}\rho \\
\left({ }^{\prime \prime}\right)\end{array}$ & $\begin{array}{l}\sigma_{\rho} \\
\left({ }^{\prime \prime}\right)\end{array}$ & $\begin{array}{c}\theta \\
\left({ }^{\circ}\right)\end{array}$ & $\begin{array}{l}\sigma_{\theta} \\
\left(^{\circ}\right)\end{array}$ & Notes & Orbit & $\begin{array}{r}\Delta \rho(\mathrm{O}-\mathrm{C}) \\
\left({ }^{\prime \prime}\right)\end{array}$ & $\begin{array}{r}\Delta \theta(\mathrm{O}-\mathrm{C}) \\
\left({ }^{\circ}\right)\end{array}$ \\
\hline $11252+1608$ & HEI156BC & - & 2016.386 & $\mathrm{R}$ & 20 & - & - & - & - & Unres. & & & \\
\hline $11258+5527$ & MLR601 & - & 2016.326 & $\mathrm{R}$ & 20 & 1.056 & 0.005 & $56.0^{*}$ & 0.3 & & & & \\
\hline $11277+4410$ & A1848 & 8169 & 2016.326 & $\mathrm{R}$ & 20 & 0.554 & 0.005 & 193.6 & 0.5 & Elongated & & & \\
\hline $11290+0105$ & RST5354 & - & 2016.301 & $\mathrm{R}$ & 20 & 0.891 & 0.011 & 287.8 & 0.7 & & & & \\
\hline $11292+0606$ & HEI851 & - & 2016.296 & $\mathrm{R}$ & 20 & 0.923 & 0.016 & 145.1 & 0.5 & & & & \\
\hline $11294+4206$ & COU1905 & - & 2016.266 & $\mathrm{R}$ & 20 & - & - & - & - & Unres. & & & \\
\hline $11308+4117$ & STT234 & 8189 & 2016.285 & $\mathrm{R}$ & 20 & 0.444 & 0.004 & 179.9 & 0.6 & Elongated & Doc $2009 \mathrm{~g}$ & 0.01 & -1.6 \\
\hline $11321+1809$ & HDS1635 & - & 2016.296 & $\mathrm{R}$ & 20 & - & - & - & - & Unres. & & & \\
\hline $11330+0938$ & $\mathrm{~A} 2576 \mathrm{AB}$ & 8211 & 2016.340 & $\mathrm{R}$ & 20 & 0.541 & 0.004 & $262.0^{*}$ & 0.5 & Faint & Sca2016a & 0.00 & -0.9 \\
\hline $11330+0938$ & A2576AB & 8211 & 2016.342 & $\mathrm{R}$ & 20 & 0.551 & 0.006 & $262.0^{*}$ & 0.3 & & Sca2016a & 0.01 & -0.9 \\
\hline $11332+4927$ & HU727 & 8210 & 2016.331 & $\mathrm{R}$ & 20 & 1.247 & 0.006 & 204.9 & 0.3 & Artifacts & & & \\
\hline $11333+5748$ & MLR551Aa,Ab & 8209 & 2016.266 & $\mathrm{R}$ & 20 & 0.948 & 0.007 & $125.0^{*}$ & 0.3 & & & & \\
\hline $11336+4729$ & COU1573 & - & 2016.326 & $\mathrm{R}$ & 20 & 0.630 & 0.006 & 89.5 & 0.3 & & & & \\
\hline $11352+3524$ & HU887 & 8225 & 2016.342 & $\mathrm{R}$ & 20 & 1.290 & 0.013 & $304.9^{*}$ & 0.3 & & & & \\
\hline $11361+1251$ & STF1554 & 8230 & 2016.329 & $\mathrm{R}$ & 20 & 0.298 & 0.004 & 214.7 & 1.5 & Elongated & Zir2011b & -0.02 & -3.2 \\
\hline $11362-0223$ & RST4946 & - & 2016.301 & $\mathrm{R}$ & 20 & 1.032 & 0.008 & 231.3 & 0.7 & & & & \\
\hline $11366+0834$ & HEI502 & - & 2016.329 & $\mathrm{R}$ & 20 & 1.020 & 0.006 & 330.9 & 0.3 & & & & \\
\hline $11367+2128$ & STF1558AB & 8238 & 2016.288 & $\mathrm{R}$ & 20 & 1.277 & 0.028 & 167.8 & 0.5 & & & & \\
\hline $11371+4040$ & A1996 & 8241 & 2016.389 & $\mathrm{R}$ & 20 & 1.991 & 0.011 & $190.2^{*}$ & 0.3 & Elongated & & & \\
\hline $11383-0029$ & RST5357 & - & 2016.301 & $\mathrm{R}$ & 20 & 0.866 & 0.004 & 36.8 & 1.1 & & & & \\
\hline $11409-0247$ & A1356 & 8267 & 2016.342 & $\mathrm{R}$ & 20 & 1.427 & 0.012 & $296.6^{*}$ & 0.3 & & & & \\
\hline $11428+2105$ & HU888 & 8275 & 2016.288 & $\mathrm{R}$ & 20 & 0.546 & 0.012 & 179.2 & 0.4 & & & & \\
\hline $11468+1500$ & BU602 & 8302 & 2016.340 & $\mathrm{R}$ & 20 & 0.536 & 0.007 & $307.8^{*}$ & 0.5 & & & & \\
\hline $11478+4949$ & HU729 & 8307 & 2016.329 & $\mathrm{R}$ & 20 & 1.501 & 0.008 & $346.6^{*}$ & 0.3 & & & & \\
\hline $11487+3937$ & COU1263 & - & 2016.329 & $\mathrm{R}$ & 20 & 0.408 & 0.008 & 240.3 & 0.4 & Faint & & & \\
\hline $11487+4030$ & COU1262 & - & 2016.329 & $\mathrm{R}$ & 20 & 0.668 & 0.004 & 248.8 & 0.3 & Elongated & & & \\
\hline $11510+3653$ & HU889 & 8319 & 2016.331 & $\mathrm{R}$ & 20 & 0.977 & 0.005 & 295.5 & 0.3 & & & & \\
\hline $11540+2239$ & TDS8101 & - & 2016.340 & $\mathrm{R}$ & 20 & - & - & - & - & & & & \\
\hline $11569+4021$ & STF1586 & 8358 & 2016.332 & $\mathrm{R}$ & 20 & 1.477 & 0.007 & 254.9 & 0.3 & & & & \\
\hline $11579+2458$ & A680 & 8365 & 2016.389 & $\mathrm{R}$ & 20 & 0.455 & 0.009 & 318.9 & 1.2 & & & & \\
\hline $11598+5324$ & STT243 & 8378 & 2016.389 & $\mathrm{R}$ & 20 & 1.197 & 0.008 & 8.5 & 0.4 & Elongated & & & \\
\hline $12006+4337$ & STF1589 & 8386 & 2016.269 & $\mathrm{R}$ & 20 & 2.214 & 0.011 & $158.3^{*}$ & 0.3 & & & & \\
\hline $12022+2108$ & A 2163 & 8396 & 2016.269 & $\mathrm{R}$ & 20 & 0.689 & 0.006 & $170.2^{*}$ & 0.4 & & & & \\
\hline $12024+1916$ & $\mathrm{~A} 2165$ & 8399 & 2016.269 & $\mathrm{R}$ & 10 & - & - & - & - & Unres. & & & \\
\hline $12056+5659$ & A1358AB & 8415 & 2016.340 & $\mathrm{R}$ & 20 & 0.533 & 0.004 & 48.9 & 0.6 & & & & \\
\hline $12061+3850$ & STF1601 & 8421 & 2016.269 & $\mathrm{R}$ & 20 & 2.013 & 0.011 & $298.0^{*}$ & 0.3 & Elongated & & & \\
\hline $12093+1525$ & A2056 & 8439 & 2016.337 & $\mathrm{R}$ & 20 & 0.583 & 0.010 & $125.7^{*}$ & 0.3 & Elongated & & & \\
\hline $12094+5758$ & TDS8247 & - & 2016.337 & $\mathrm{R}$ & 20 & 0.555 & 0.007 & 325.8 & 0.3 & & & & \\
\hline $12111+1228$ & HDS1719 & - & 2016.337 & $\mathrm{R}$ & 20 & 0.559 & 0.007 & $275.8^{*}$ & 0.7 & & & & \\
\hline $12120+1300$ & A1780 & 8456 & 2016.337 & $\mathrm{R}$ & 20 & 1.252 & 0.008 & $135.6^{*}$ & 0.3 & Elongated & & & \\
\hline $12160+4807$ & HU736 & 8485 & 2016.329 & $\mathrm{R}$ & 20 & 0.367 & 0.004 & $181.7^{*}$ & 0.3 & & USN2006a & 0.04 & 0.0 \\
\hline $12167+3004$ & AG176 & 8496 & 2016.397 & $\mathrm{R}$ & 20 & 2.360 & 0.012 & $3.5^{*}$ & 0.3 & Elongated & & & \\
\hline $12189+5622$ & STF1630 & 8507 & 2016.296 & $\mathrm{R}$ & 20 & 2.524 & 0.031 & $170.3^{*}$ & 0.7 & & & & \\
\hline $12194+1744$ & A2059 & 8508 & 2016.340 & $\mathrm{R}$ & 20 & 0.453 & 0.004 & $219.4^{*}$ & 1.6 & & $\operatorname{Lin} 2017 \mathrm{a}$ & 0.01 & $-1.3^{Q}$ \\
\hline $12238+5410$ & STT249AB & 8535 & 2016.296 & $\mathrm{R}$ & 20 & 0.388 & 0.006 & 257.1 & 2.3 & & & & \\
\hline $12244+4305$ & STT250 & 8540 & 2016.340 & $\mathrm{R}$ & 10 & 0.301 & 0.009 & $353.2^{*}$ & 1.4 & & & & \\
\hline $12265-0153$ & HLD13AB & 8549 & 2016.389 & $\mathrm{R}$ & 20 & 1.324 & 0.007 & 133.9 & 0.3 & Elongated & & & \\
\hline $12268-0011$ & AGC4 & 8550 & 2016.329 & $\mathrm{R}$ & 20 & 0.530 & 0.008 & $144.7^{*}$ & 0.5 & & & & \\
\hline $12291+3123$ & STT251 & 8569 & 2016.288 & $\mathrm{R}$ & 20 & 0.739 & 0.014 & $59.5^{*}$ & 1.0 & & Sca2003c & 0.06 & -2.0 \\
\hline $12295+2931$ & BU1324AB & 8570 & 2016.397 & $\mathrm{R}$ & 20 & 2.402 & 0.012 & $224.5^{*}$ & 0.3 & & & & \\
\hline $12306+0943$ & STF1647 & 8575 & 2016.285 & $\mathrm{R}$ & 20 & 1.289 & 0.009 & 248.6 & 0.7 & & Hop1970 & 0.05 & -3.3 \\
\hline $12329+5448$ & A1600 & 8594 & 2016.291 & $\mathrm{R}$ & 20 & 0.880 & 0.004 & 8.4 & 0.4 & Diffuse & & & \\
\hline $12334+3410$ & HO537 & 8596 & 2016.397 & $\mathrm{R}$ & 20 & 2.429 & 0.012 & $174.4^{*}$ & 0.3 & & & & \\
\hline $12340+2650$ & COU595 & - & 2016.340 & $\mathrm{R}$ & 20 & 0.993 & 0.006 & $2.3^{*}$ & 0.3 & & & & \\
\hline
\end{tabular}


Table 2 Table of speckle measurements and O-C residuals with published orbits (cont.)

\begin{tabular}{|c|c|c|c|c|c|c|c|c|c|c|c|c|c|}
\hline WDS & Name & ADS & Epoch & Fil. & $\begin{array}{l}\text { Eyep. } \\
(\mathrm{mm})\end{array}$ & $\begin{array}{c}\rho \\
\left({ }^{\prime \prime}\right)\end{array}$ & $\begin{array}{l}\sigma_{\rho} \\
\left({ }^{\prime \prime}\right)\end{array}$ & $\begin{array}{c}\theta \\
\left({ }^{\circ}\right)\end{array}$ & $\begin{array}{l}\sigma_{\theta} \\
\left(^{\circ}\right)\end{array}$ & Notes & Orbit & $\begin{array}{r}\Delta \rho(\mathrm{O}-\mathrm{C}) \\
\left({ }^{\prime \prime}\right)\end{array}$ & $\begin{array}{r}\Delta \theta(\mathrm{O}-\mathrm{C}) \\
\left(^{\circ}\right)\end{array}$ \\
\hline $12345+0558$ & BU797AB & 8598 & 2016.288 & $\mathrm{R}$ & 20 & 0.619 & 0.018 & 145.9 & 0.5 & & & & \\
\hline $12349+2238$ & WRH12 & - & 2016.288 & $\mathrm{R}$ & 10 & 0.340 & 0.008 & $12.0^{*}$ & ${ }^{*} 1.0$ & & Hrt2014b & -0.01 & 0.2 \\
\hline $12349+2238$ & WRH12 & - & 2016.288 & $\mathrm{R}$ & 10 & 0.341 & 0.003 & $12.0^{*}$ & * 0.9 & & Hrt2014b & -0.01 & 0.2 \\
\hline $12372+2112$ & STF1663 & 8611 & 2016.291 & $\mathrm{R}$ & 20 & 0.636 & 0.004 & $66.5^{*}$ & ${ }^{*} 0.3$ & & Zir2013a & 0.00 & -1.0 \\
\hline $12417-0127$ & STF1670AB & 8630 & 2016.266 & $\mathrm{R}$ & 20 & 2.452 & 0.042 & 3.7 & 0.3 & & Sca2007c & 0.00 & 0.3 \\
\hline $12429+0516$ & A1602 & 8639 & 2016.386 & $\mathrm{R}$ & 20 & 0.598 & 0.004 & 27.2 & 1.0 & & Doc2015d & -0.00 & 0.4 \\
\hline $12437+4942$ & TDS8505 & - & 2016.386 & $\mathrm{R}$ & 20 & 0.505 & 0.004 & 174.6 & 0.9 & & & & \\
\hline $12441+3546$ & HO256 & 8651 & 2016.296 & $\mathrm{R}$ & 20 & 0.522 & 0.004 & $120.4^{*}$ & * 0.5 & & & & \\
\hline $12454+0331$ & $\mathrm{~J} 432$ & 8658 & 2016.386 & $\mathrm{R}$ & 20 & 1.216 & 0.006 & 249.3 & 0.3 & & & & \\
\hline $12501+2408$ & A563 & 8677 & 2016.389 & $\mathrm{R}$ & 20 & 0.400 & 0.004 & 180.1 & 0.6 & & & & \\
\hline $12507+2032$ & HU640AB & 8680 & 2016.389 & $\mathrm{R}$ & 20 & 0.444 & 0.004 & 250.2 & 0.5 & & Hrt2014b & 0.02 & 1.8 \\
\hline $12510+5959$ & HU1140 & 8683 & 2016.302 & $\mathrm{R}$ & 20 & 0.615 & 0.006 & 261.3 & 0.3 & & & & \\
\hline $12515+2714$ & ES439 & 8686 & 2016.397 & $\mathrm{R}$ & 20 & 2.111 & 0.011 & $67.4^{*}$ & * 0.4 & & & & \\
\hline $12526+2342$ & COU396 & - & 2016.302 & $\mathrm{R}$ & 20 & 0.884 & 0.011 & 314.5 & 0.5 & Elongated & & & \\
\hline $12533+2115$ & STF1687AB & 8695 & 2016.291 & $\mathrm{R}$ & 20 & 1.179 & 0.006 & $199.2^{*}$ & ${ }^{*} 0.4$ & Elongated & Dru2014 & 0.01 & 0.5 \\
\hline $12547+2206$ & MET9 & - & 2016.302 & $\mathrm{R}$ & 20 & 1.685 & 0.008 & $50.4^{*}$ & * 0.3 & & & & \\
\hline $12549+0919$ & HEI514 & - & 2016.389 & $\mathrm{R}$ & 20 & 1.339 & 0.007 & $87.2^{*}$ & ${ }^{*} 0.3$ & & & & \\
\hline $12556+5706$ & MLR553 & - & 2016.296 & $\mathrm{R}$ & 20 & 0.476 & 0.006 & 187.5 & 1.3 & & & & \\
\hline $12560+5305$ & TDS8595 & - & 2016.302 & $\mathrm{R}$ & 20 & 0.624 & 0.006 & $225.1^{*}$ & ${ }^{*} 0.4$ & & & & \\
\hline $12563+4300$ & A 2000 & 8709 & 2016.296 & $\mathrm{R}$ & 20 & 1.018 & 0.005 & 48.8 & 1.8 & & & & \\
\hline $12575+2457$ & COU397 & - & 2016.296 & $\mathrm{R}$ & 20 & 0.708 & 0.006 & 60.3 & 1.7 & Elongated & & & \\
\hline $12587+2728$ & STF1699 & 8721 & 2016.291 & $\mathrm{R}$ & 20 & 1.704 & 0.009 & 8.9 & 0.3 & & & & \\
\hline $12595+3004$ & A1852 & 8726 & 2016.411 & $\mathrm{R}$ & 20 & 0.977 & 0.012 & 310.9 & 0.3 & & & & \\
\hline $13007+5622$ & BU1082 & 8739 & 2016.266 & $\mathrm{R}$ & 20 & 0.816 & 0.005 & 123.6 & 0.3 & & Sca2012c & -0.01 & 2.9 \\
\hline $13007+5622$ & BU1082 & 8739 & 2016.378 & $\mathrm{R}$ & 20 & 0.820 & 0.006 & $124.4^{*}$ & ${ }^{*} 0.3$ & & Sca2012c & -0.00 & 3.2 \\
\hline $13041+5227$ & MLR704 & - & 2016.340 & $\mathrm{R}$ & 20 & 0.410 & 0.004 & $217.8^{*}$ & ${ }^{*} 0.4$ & & & & \\
\hline $13042+5615$ & MLR554 & - & 2016.266 & $\mathrm{R}$ & 20 & 0.606 & 0.015 & $144.7^{*}$ & * 0.9 & & & & \\
\hline $13069+5200$ & A1605 & 8785 & 2016.340 & $\mathrm{R}$ & 20 & 1.087 & 0.005 & 349.7 & 0.4 & & & & \\
\hline $13081+2657$ & STT260 & 8791 & 2016.326 & $\mathrm{R}$ & 20 & 0.311 & 0.004 & 220.6 & 0.4 & Elongated & Zir2008 & -0.04 & $-36.5^{Q}$ \\
\hline $13091+2127$ & HU572 & 8799 & 2016.321 & $\mathrm{R}$ & 20 & 0.511 & 0.004 & 334.4 & 0.6 & & Fmr2012b & -0.02 & 1.0 \\
\hline $13097+4418$ & COU1909 & - & 2016.321 & $\mathrm{R}$ & 20 & 0.649 & 0.008 & 252.1 & 0.7 & & & & \\
\hline $13101+3830$ & BU608BC & 8805 & 2016.291 & $\mathrm{R}$ & 20 & 1.254 & 0.006 & $268.9^{*}$ & ${ }^{*} 0.3$ & & & & \\
\hline $13121+3655$ & STF1730 & - & 2016.414 & $\mathrm{R}$ & 20 & 1.871 & 0.009 & 338.7 & 0.4 & & & & \\
\hline $13128+4030$ & A1606 & 8820 & 2016.291 & $\mathrm{R}$ & 20 & 1.296 & 0.006 & 15.7 & 0.3 & & & & \\
\hline $13134+5252$ & A1607 & 8825 & 2016.321 & $\mathrm{R}$ & 20 & 0.477 & 0.004 & 9.1 & 0.3 & & & & \\
\hline $13157+5424$ & HDS1858 & - & 2016.340 & $\mathrm{R}$ & 20 & 0.530 & 0.008 & 172.2 & 0.9 & & & & \\
\hline $13177+5845$ & A1360 & 8851 & 2016.326 & $\mathrm{R}$ & 20 & 0.784 & 0.004 & $142.0^{*}$ & ${ }^{*} 0.3$ & & & & \\
\hline $13198+4747$ & HU644AB & 8862 & 2016.269 & $\mathrm{R}$ & 20 & 0.587 & 0.006 & $83.0^{*}$ & ${ }^{*} 0.7$ & & Msn2012a & 0.02 & 0.3 \\
\hline $13221+2755$ & MLB646 & - & 2016.337 & $\mathrm{R}$ & 20 & 2.522 & 0.013 & 163.1 & 0.3 & & & & \\
\hline $13222+2710$ & A565 & 8875 & 2016.337 & $\mathrm{R}$ & 20 & 0.785 & 0.004 & $196.0^{*}$ & * 0.8 & & & & \\
\hline $13223+2631$ & A566 & 8876 & 2016.337 & $\mathrm{R}$ & 20 & 1.966 & 0.010 & $245.5^{*}$ & * 0.3 & Elongated & & & \\
\hline $13232+4029$ & POP119 & - & 2016.326 & $\mathrm{R}$ & 20 & 0.750 & 0.006 & 4.8 & 0.5 & & & & \\
\hline $13237-0043$ & A2489 & 8884 & 2016.321 & $\mathrm{R}$ & 20 & 0.986 & 0.005 & 190.7 & 0.6 & & WSI2004a & 0.02 & -0.8 \\
\hline $13252+1518$ & J749 & 8894 & 2016.419 & $\mathrm{R}$ & 20 & 2.653 & 0.013 & 286.7 & 0.3 & & & & \\
\hline $13258+4430$ & A1609AB & 8901 & 2016.291 & $\mathrm{R}$ & 20 & 0.361 & 0.006 & 57.8 & 1.1 & & Hei1991 & 0.01 & -1.3 \\
\hline $13258+4430$ & A1609AC & 8901 & 2016.291 & $\mathrm{R}$ & 20 & 2.500 & 0.012 & 39.7 & 0.3 & & & & \\
\hline $13258+4430$ & A1609BC & 8901 & 2016.291 & $\mathrm{R}$ & 20 & 2.849 & 0.014 & 41.9 & 0.3 & & & & \\
\hline $13262+0345$ & HEI773 & - & 2016.302 & $\mathrm{R}$ & 20 & 0.883 & 0.007 & $72.0^{*}$ & ${ }^{*} 0.3$ & & & & \\
\hline $13268+5043$ & COU2190 & - & 2016.302 & $\mathrm{R}$ & 20 & 0.435 & 0.004 & $37.9^{*}$ & ${ }^{*} 0.4$ & & & & \\
\hline $13280+3235$ & A1856 & 8911 & 2016.334 & $\mathrm{R}$ & 20 & 1.072 & 0.007 & $344.4^{*}$ & ${ }^{*} 0.3$ & & & & \\
\hline $13283+0214$ & A2490 & 8913 & 2016.302 & $\mathrm{R}$ & 20 & 1.177 & 0.006 & $91.4^{*}$ & * 0.3 & & & & \\
\hline $13305+3430$ & A1857 & 8927 & 2016.296 & $\mathrm{R}$ & 20 & 0.440 & 0.007 & $341.6^{*}$ & * 0.6 & & & & \\
\hline $13315+5725$ & MLR555 & - & 2016.302 & $\mathrm{R}$ & 20 & 0.554 & 0.004 & $169.0^{*}$ & ${ }^{*} 0.4$ & & & & \\
\hline $13328+1649$ & VYS6 & - & 2016.288 & $\mathrm{R}$ & 20 & 2.686 & 0.013 & $54.2^{*}$ & ${ }^{*} 0.9$ & & Hei1990d & -0.05 & 0.8 \\
\hline
\end{tabular}


Table 2 Table of speckle measurements and O-C residuals with published orbits (cont.)

\begin{tabular}{|c|c|c|c|c|c|c|c|c|c|c|c|c|c|}
\hline WDS & Name & ADS & Epoch & Fil. & $\begin{array}{l}\text { Eyep. } \\
(\mathrm{mm})\end{array}$ & $\begin{array}{c}\rho \\
\left({ }^{\prime \prime}\right)\end{array}$ & $\begin{array}{l}\sigma_{\rho} \\
\left({ }^{\prime \prime}\right)\end{array}$ & $\begin{array}{c}\theta \\
\left({ }^{\circ}\right)\end{array}$ & $\begin{array}{l}\sigma_{\theta} \\
\left(^{\circ}\right)\end{array}$ & Notes & Orbit & $\begin{array}{r}\Delta \rho(\mathrm{O}-\mathrm{C}) \\
\left({ }^{\prime \prime}\right)\end{array}$ & $\begin{array}{r}\Delta \theta(\mathrm{O}-\mathrm{C}) \\
\left(^{\circ}\right)\end{array}$ \\
\hline $13328+2421$ & A567 & 8937 & 2016.340 & $\mathrm{R}$ & 20 & 1.453 & 0.008 & $255.6^{*}$ & 0.3 & Faint & & & \\
\hline $13331+4316$ & COU1754 & - & 2016.402 & $\mathrm{R}$ & 10 & - & - & - & - & Unres. & & & \\
\hline $13333+4526$ & A1610 & 8942 & 2016.334 & $\mathrm{R}$ & 20 & 0.907 & 0.005 & 267.4 & 0.4 & Elongated & & & \\
\hline $13336+2944$ & A1095 & 8943 & 2016.296 & $\mathrm{R}$ & 20 & 0.339 & 0.007 & 288.0 & 0.8 & & Ole2002a & 0.01 & -1.0 \\
\hline $13340+0847$ & A1792 & 8946 & 2016.288 & $\mathrm{R}$ & 20 & 0.722 & 0.017 & $308.2^{*}$ & 0.9 & & & & \\
\hline $13368+0650$ & A1611 & 8968 & 2016.288 & $\mathrm{R}$ & 20 & 0.882 & 0.022 & 121.7 & 0.8 & & & & \\
\hline $13377+5028$ & BU934 & 8978 & 2016.378 & $\mathrm{R}$ & 20 & 1.264 & 0.006 & 263.5 & 0.3 & & & & \\
\hline $13377+5043$ & STF 1770 & 8979 & 2016.378 & $\mathrm{R}$ & 20 & 1.713 & 0.009 & $122.5^{*}$ & 0.3 & & & & \\
\hline $13400+3759$ & HU897AB & 8988 & 2016.326 & $\mathrm{R}$ & 20 & 0.353 & 0.004 & $57.9^{*}$ & 0.6 & & Zir2015a & -0.01 & 1.6 \\
\hline $13442+5921$ & HU1261 & 9009 & 2016.378 & $\mathrm{R}$ & 20 & 1.073 & 0.007 & $101.3^{*}$ & 0.3 & & & & \\
\hline $13453+4559$ & COU1915 & - & 2016.326 & $\mathrm{R}$ & 20 & 1.632 & 0.008 & $76.1^{*}$ & ${ }^{*} 0.3$ & & & & \\
\hline $13478+0120$ & A2492 & 9026 & 2016.378 & $\mathrm{R}$ & 20 & 0.948 & 0.005 & 199.4 & 0.8 & Elongated & & & \\
\hline $13485-0052$ & RST4991 & - & 2016.378 & $\mathrm{R}$ & 20 & 1.333 & 0.007 & 290.3 & 0.3 & & & & \\
\hline $13532-0333$ & RST5530 & - & 2016.430 & $\mathrm{R}$ & 20 & 0.891 & 0.007 & 20.5 & 0.3 & Diffuse & & & \\
\hline $13577+5200$ & A1614 & 9071 & 2016.384 & $\mathrm{R}$ & 20 & 1.425 & 0.007 & 299.7 & 0.3 & & RAO2015 & 0.03 & 0.1 \\
\hline $14009+0912$ & HEI777 & - & 2016.326 & $\mathrm{R}$ & 20 & 1.763 & 0.009 & 215.3 & 0.3 & Faint & & & \\
\hline $14012+2522$ & A569 & 9084 & 2016.389 & $\mathrm{R}$ & 20 & 0.589 & 0.005 & 149.2 & 0.4 & & & & \\
\hline $14020+5713$ & A1097AB & 9089 & 2016.296 & $\mathrm{R}$ & 20 & 0.405 & 0.011 & $259.4^{*}$ & * 1.6 & & Sca2000a & -0.02 & 3.4 \\
\hline $14028+1417$ & HEI65 & - & 2016.397 & $\mathrm{R}$ & 20 & 0.310 & 0.004 & 94.1 & 0.4 & & & & \\
\hline $14034+1740$ & A2064 & 9093 & 2016.397 & $\mathrm{R}$ & 20 & 1.060 & 0.006 & 176.9 & 0.3 & & & & \\
\hline $14037+0829$ & BU1270 & 9094 & 2016.430 & $\mathrm{R}$ & 10 & 0.222 & 0.002 & 4.6 & 0.8 & & USN2006b & -0.01 & -1.2 \\
\hline $14065-0249$ & RST4523 & - & 2016.389 & $\mathrm{R}$ & 20 & 0.379 & 0.004 & 81.2 & 0.4 & & & & \\
\hline $14078+2443$ & A346 & 9116 & 2016.397 & $\mathrm{R}$ & 20 & 0.967 & 0.008 & 308.7 & 0.3 & & & & \\
\hline $14082+3645$ & STT276AB & 9121 & 2016.321 & $\mathrm{R}$ & 20 & 0.425 & 0.004 & 209.0 & 0.5 & & & & \\
\hline $14110+0525$ & TDS9122 & - & 2016.389 & $\mathrm{R}$ & 20 & - & - & - & - & Unres. & & & \\
\hline $14122+2722$ & $\mathrm{~J} 1125$ & 9154 & 2016.386 & $\mathrm{R}$ & 20 & 0.682 & 0.005 & 96.3 & 0.3 & Elongated & & & \\
\hline $14122+4411$ & STT278 & 9159 & 2016.340 & $\mathrm{R}$ & 10 & 0.368 & 0.004 & $93.5^{*}$ & * 0.6 & & Hrt2009 & -0.02 & $-0.5^{Q}$ \\
\hline $14131+5520$ & STF1820 & 9167 & 2016.329 & $\mathrm{R}$ & 20 & 2.765 & 0.014 & 123.0 & 0.3 & & Kiy1998 (orb.1) & 0.09 & -0.3 \\
\hline $14135+1234$ & BU224 & 9165 & 2016.329 & $\mathrm{R}$ & 20 & 0.632 & 0.009 & $100.6^{*}$ & * 0.3 & & Lin1985c & -0.02 & 1.4 \\
\hline $14139+2906$ & STF1816 & 9174 & 2016.291 & $\mathrm{R}$ & 20 & 0.400 & 0.006 & $98.9^{*}$ & ${ }^{*} 0.4$ & & Zir2014a & 0.02 & 0.4 \\
\hline $14141+1056$ & HEI530 & - & 2016.386 & $\mathrm{R}$ & 20 & 0.538 & 0.008 & 353.3 & 0.3 & & & & \\
\hline $14145-0459$ & A 2588 & 9176 & 2016.386 & $\mathrm{R}$ & 20 & 0.952 & 0.005 & 220.2 & 0.5 & & & & \\
\hline $14148+0015$ & RST5383 & - & 2016.329 & $\mathrm{R}$ & 20 & 0.548 & 0.004 & 44.2 & 0.7 & Elongated & & & \\
\hline $14151-0125$ & RST4997 & - & 2016.329 & $\mathrm{R}$ & 20 & 0.282 & 0.008 & 250.4 & 0.7 & Elongated & & & \\
\hline $14153+0308$ & STF1819AB & 9182 & 2016.266 & $\mathrm{R}$ & 20 & 0.893 & 0.011 & 165.9 & 0.3 & & Sca2012b & 0.01 & 0.8 \\
\hline $14153+0308$ & STF1819AB & 9182 & 2016.387 & $\mathrm{R}$ & 20 & 0.899 & 0.012 & 165.6 & 0.3 & Elongated & Sca2012b & 0.01 & 0.8 \\
\hline $14160+5247$ & A1616 & 9194 & 2016.266 & $\mathrm{R}$ & 20 & 1.644 & 0.008 & $84.4^{*}$ & ${ }^{*} 0.4$ & & & & \\
\hline $14171+4529$ & A1617 & 9205 & 2016.430 & $\mathrm{R}$ & 20 & 0.458 & 0.004 & 281.4 & 0.8 & & & & \\
\hline $14171+5100$ & A 147 & 9206 & 2016.296 & $\mathrm{R}$ & 20 & 0.618 & 0.012 & $106.8^{*}$ & ${ }^{*} 1.2$ & & & & \\
\hline $14171+5100$ & A147 & 9206 & 2016.340 & $\mathrm{R}$ & 20 & 0.607 & 0.006 & $106.7^{*}$ & * 0.3 & & & & \\
\hline $14175+1722$ & A2067 & 9204 & 2016.386 & $\mathrm{R}$ & 10 & - & - & - & - & Unres. & & & \\
\hline $14184+3412$ & HU901 & 9214 & 2016.288 & $\mathrm{R}$ & 20 & 0.632 & 0.005 & 35.7 & 1.7 & & & & \\
\hline $14185+4755$ & BU1273 & 9217 & 2016.430 & $\mathrm{R}$ & 20 & 1.328 & 0.014 & 176.7 & 0.3 & Diffuse & & & \\
\hline $14188+5934$ & HU1267 & 9221 & 2016.302 & $\mathrm{R}$ & 20 & 0.753 & 0.004 & $185.8^{*}$ & * 0.3 & & & & \\
\hline $14188+5934$ & HU1267 & 9221 & 2016.337 & $\mathrm{R}$ & 20 & 0.752 & 0.004 & $6.0^{*}$ & * 0.3 & & & & \\
\hline $14205+2634$ & DAN1 & - & 2016.403 & $\mathrm{R}$ & 10 & - & - & - & - & Unres. & & & \\
\hline $14213+3050$ & COU482 & - & 2016.397 & $\mathrm{R}$ & 20 & 0.629 & 0.005 & 121.1 & 0.3 & Elongated & & & \\
\hline $14220+5107$ & A148 & 9238 & 2016.403 & $\mathrm{R}$ & 20 & 0.501 & 0.005 & 190.3 & 0.4 & & & & \\
\hline $14234+4736$ & $\mathrm{~A} 149 \mathrm{AB}$ & 9249 & 2016.291 & $\mathrm{R}$ & 20 & 0.784 & 0.007 & 123.3 & 0.3 & & & & \\
\hline $14241+4331$ & COU1916 & - & 2016.430 & $\mathrm{R}$ & 20 & 0.745 & 0.006 & 268.3 & 0.4 & & & & \\
\hline $14303+4709$ & COU1917 & - & 2016.337 & $\mathrm{R}$ & 20 & 0.426 & 0.004 & 40.7 & 0.3 & & & & \\
\hline $14333+2725$ & A688 & 9308 & 2016.337 & $\mathrm{R}$ & 20 & 0.759 & 0.004 & $195.4^{*}$ & * 0.4 & & & & \\
\hline $14339+5514$ & STF1860 & 9315 & 2016.403 & $\mathrm{R}$ & 20 & 1.047 & 0.005 & 112.7 & 0.3 & & & & \\
\hline $14340-0507$ & A2589 & 9309 & 2016.386 & $\mathrm{R}$ & 20 & 1.026 & 0.008 & 202.7 & 0.9 & Elongated & & & \\
\hline
\end{tabular}


Table 2 Table of speckle measurements and O-C residuals with published orbits (cont.)

\begin{tabular}{|c|c|c|c|c|c|c|c|c|c|c|c|c|c|}
\hline WDS & Name & ADS & Epoch & Fil. & $\begin{array}{l}\text { Eyep. } \\
(\mathrm{mm})\end{array}$ & $\begin{array}{c}\rho \\
\left({ }^{\prime \prime}\right)\end{array}$ & $\begin{array}{l}\sigma_{\rho} \\
\left({ }^{\prime \prime}\right)\end{array}$ & $\begin{array}{c}\theta \\
\left({ }^{\circ}\right)\end{array}$ & $\begin{array}{l}\sigma_{\theta} \\
\left(^{\circ}\right)\end{array}$ & Notes & Orbit & $\begin{array}{r}\Delta \rho(\mathrm{O}-\mathrm{C}) \\
\left({ }^{\prime \prime}\right)\end{array}$ & $\begin{array}{r}\Delta \theta(\mathrm{O}-\mathrm{C}) \\
\left({ }^{\circ}\right)\end{array}$ \\
\hline $14369+4813$ & A 347 & 9324 & 2016.329 & $\mathrm{R}$ & 20 & 0.551 & 0.004 & $236.4^{*}$ & 1.1 & Elongated & Doc2017k & -0.00 & -0.3 \\
\hline $14380+5135$ & STF1863 & 9329 & 2016.329 & $\mathrm{R}$ & 20 & 0.668 & 0.004 & $59.9^{*}$ & 0.7 & & Zir2013a & 0.02 & -0.0 \\
\hline $14380+5135$ & STF1863 & 9329 & 2016.403 & $\mathrm{R}$ & 20 & 0.665 & 0.004 & 59.8 & 0.4 & & Zir2013a & 0.02 & -0.1 \\
\hline $14407+3117$ & STF1867 & 9340 & 2016.329 & $\mathrm{R}$ & 20 & 0.668 & 0.004 & $353.2^{*}$ & 0.4 & & Zir2013a & 0.02 & 0.0 \\
\hline $14407+3117$ & STF1867 & 9340 & 2016.403 & $\mathrm{R}$ & 20 & 0.666 & 0.004 & 353.3 & 0.3 & & Zir2013a & 0.01 & 0.1 \\
\hline $14416+2747$ & COU407 & - & 2016.337 & $\mathrm{R}$ & 20 & 0.424 & 0.008 & 112.6 & 0.5 & & & & \\
\hline $14441+3138$ & COU607 & - & 2016.387 & $\mathrm{R}$ & 20 & 0.723 & 0.011 & 314.9 & 0.5 & & & & \\
\hline $14455+4223$ & STT285 & 9378 & 2016.403 & $\mathrm{R}$ & 20 & 0.499 & 0.004 & $79.8^{*}$ & ${ }^{k} 0.4$ & & Sca2015b & 0.00 & -0.0 \\
\hline $14463-0117$ & RST5006 & - & 2016.329 & $\mathrm{R}$ & 20 & 0.647 & 0.004 & 209.9 & 0.3 & Elongated & & & \\
\hline $14470-0049$ & RST5007 & - & 2016.329 & $\mathrm{R}$ & 20 & 0.506 & 0.011 & $333.3^{*}$ & 0.4 & & & & \\
\hline $14484+2422$ & STF1884 & 9389 & 2016.376 & $\mathrm{R}$ & 20 & 2.165 & 0.011 & $55.6^{*}$ & 0.3 & Elongated & & & \\
\hline $14515+4456$ & STT287 & 9418 & 2016.403 & $\mathrm{R}$ & 20 & 0.613 & 0.004 & $3.5^{*}$ & 0.3 & & Hei1997 & -0.09 & 2.0 \\
\hline $14534+1542$ & STT288 & 9425 & 2016.504 & $\mathrm{R}$ & 20 & 1.004 & 0.008 & 158.4 & 0.3 & Elongated & Hei1998 & 0.01 & 0.6 \\
\hline $14558+3939$ & A1627 & 9441 & 2016.403 & $\mathrm{R}$ & 10 & 0.214 & 0.003 & 199.7 & 0.8 & & Baz1993a & -0.02 & 2.3 \\
\hline $14590+1732$ & A2072 & 9463 & 2016.389 & $\mathrm{R}$ & 20 & 0.703 & 0.004 & $233.6^{*}$ & 1.2 & & & & \\
\hline $14595+1753$ & COU188 & - & 2016.419 & $\mathrm{R}$ & 20 & 0.258 & 0.005 & 229.2 & 0.7 & & & & \\
\hline $15009+5745$ & MLR602 & - & 2016.302 & $\mathrm{R}$ & 10 & 0.130 & 0.003 & 213.8 & 0.7 & & & & \\
\hline $15018-0008$ & BU348AB & 9480 & 2016.515 & $\mathrm{R}$ & 20 & 0.478 & 0.006 & 107.6 & 0.5 & Elongated & & & \\
\hline $15045+1950$ & HU745 & 9495 & 2016.493 & $\mathrm{R}$ & 20 & 0.558 & 0.008 & 18.7 & 0.6 & & & & \\
\hline $15054+1338$ & HEI378 & - & 2016.419 & $\mathrm{R}$ & 20 & 1.596 & 0.008 & 350.5 & 0.3 & & & & \\
\hline $15058+5841$ & A1114 & 9503 & 2016.493 & $\mathrm{R}$ & 20 & 1.215 & 0.008 & 285.2 & 0.5 & & & & \\
\hline $15075+5516$ & HU143Aa,Ab & 9511 & 2016.493 & $\mathrm{R}$ & 20 & 0.430 & 0.006 & $316.9^{*}$ & 0.3 & & & & \\
\hline $15077+1158$ & STF1911 & 9508 & 2016.419 & $\mathrm{R}$ & 20 & 1.948 & 0.010 & 291.8 & 0.3 & & & & \\
\hline $15078+3956$ & COU1271 & - & 2016.378 & $\mathrm{R}$ & 20 & 0.404 & 0.006 & $193.0^{*}$ & 0.8 & Elongated & Cou1999b & 0.02 & -33.6 \\
\hline $15092+2809$ & A690 & 9526 & 2016.337 & $\mathrm{R}$ & 10 & - & - & - & - & & & & \\
\hline $15100+2807$ & TDS768 & - & 2016.337 & $\mathrm{R}$ & 20 & 0.893 & 0.006 & $192.3^{*}$ & 0.3 & Elongated & & & \\
\hline $15126+2041$ & COU190 & - & 2016.378 & $\mathrm{R}$ & 20 & 1.350 & 0.007 & 272.1 & 0.3 & Elongated & & & \\
\hline $15143+1959$ & COU27AB & - & 2016.419 & $\mathrm{R}$ & 20 & - & - & - & - & Unres. & & & \\
\hline $15149+3818$ & STF1926 & 9553 & 2016.378 & $\mathrm{R}$ & 20 & 0.318 & 0.004 & $221.7^{*}$ & 0.5 & & & & \\
\hline $15183+2650$ & STF1932AB & 9578 & 2016.504 & $\mathrm{R}$ & 20 & 1.605 & 0.008 & 265.8 & 0.3 & & Sca2015b & -0.02 & 0.0 \\
\hline $15192+4329$ & A1630 & 9589 & 2016.326 & $\mathrm{R}$ & 20 & 0.787 & 0.004 & $244.4^{*}$ & 0.3 & & & & \\
\hline $15200+2338$ & COU103 & - & 2016.378 & $\mathrm{R}$ & 20 & 0.556 & 0.010 & 283.1 & 0.7 & & & & \\
\hline $15202+5824$ & MLR533 & - & 2016.302 & $\mathrm{R}$ & 20 & 0.286 & 0.004 & 311.3 & 0.3 & & & & \\
\hline $15204+5309$ & HU147 & 9602 & 2016.302 & $\mathrm{R}$ & 20 & 0.572 & 0.004 & $287.0^{*}$ & 0.3 & & & & \\
\hline $15206+1523$ & HU1160 & 9595 & 2016.337 & $\mathrm{R}$ & 20 & 0.474 & 0.006 & 175.1 & 0.8 & & Hrt2009 & 0.01 & 4.8 \\
\hline $15208+3459$ & HO62 & 9599 & 2016.504 & $\mathrm{R}$ & 20 & 1.494 & 0.019 & 103.6 & 0.4 & & & & \\
\hline $15210+2104$ & HU146 & 9600 & 2016.520 & $\mathrm{R}$ & 20 & 0.701 & 0.004 & 124.6 & 0.5 & & Zir2015a & -0.02 & 1.2 \\
\hline $15225+5516$ & HU148 & 9618 & 2016.430 & $\mathrm{R}$ & 20 & 1.507 & 0.008 & 206.8 & 0.3 & & & & \\
\hline $15232+3017$ & STF1937AB & 9617 & 2016.512 & $\mathrm{R}$ & 20 & 0.567 & 0.004 & 219.0 & 1.3 & Elongated & Wsi2006b & -0.00 & -1.7 \\
\hline $15241+2814$ & TDS9506 & - & 2016.378 & $\mathrm{R}$ & 20 & 0.424 & 0.010 & 30.5 & 0.7 & Uncertain & & & \\
\hline $15245+3723$ & STF1938Ba,Bb & 9626 & 2016.387 & $\mathrm{R}$ & 20 & 2.262 & 0.011 & $3.7^{*}$ & 0.3 & & Sca2013a & 0.05 & 0.4 \\
\hline $15257+2638$ & STF1941 & 9630 & 2016.520 & $\mathrm{R}$ & 20 & 1.382 & 0.007 & 211.9 & 0.3 & & & & \\
\hline $15271+2355$ & A 82 & 9641 & 2016.378 & $\mathrm{R}$ & 20 & 0.840 & 0.013 & 353.6 & 0.8 & Elongated & & & \\
\hline $15272+4133$ & COU1443 & - & 2016.493 & $\mathrm{R}$ & 20 & 0.446 & 0.017 & 170.5 & 0.3 & & & & \\
\hline $15272+4133$ & COU1443 & - & 2016.493 & $\mathrm{R}$ & 20 & 0.446 & 0.017 & 170.5 & 0.3 & & & & \\
\hline $15277+0606$ & STF1944 & 9647 & 2016.501 & $\mathrm{R}$ & 20 & 0.636 & 0.010 & 294.7 & 0.3 & & Zir2015a & 0.01 & 0.4 \\
\hline $15328+1945$ & HU577 & 9692 & 2016.501 & $\mathrm{R}$ & 20 & 0.347 & 0.005 & 25.1 & 1.8 & & Cou1984b & 0.01 & 0.9 \\
\hline $15329+3122$ & COU610 & - & 2016.520 & $\mathrm{R}$ & 20 & 0.844 & 0.004 & 197.8 & 0.4 & Elongated & & & \\
\hline $15360+3948$ & STT298AB & 9716 & 2016.515 & $\mathrm{R}$ & 20 & 1.199 & 0.006 & 186.1 & 0.3 & Elongated & Sod1999 & -0.01 & 0.7 \\
\hline $15361+4849$ & HU652 & 9718 & 2016.512 & $\mathrm{R}$ & 20 & 1.156 & 0.006 & 185.6 & 0.3 & & & & \\
\hline $15361+5531$ & A1124 & 9720 & 2016.531 & $\mathrm{R}$ & 20 & 1.454 & 0.007 & 142.7 & 0.3 & & & & \\
\hline $15368-0438$ & RST4545 & - & 2016.340 & $\mathrm{R}$ & 20 & 0.526 & 0.008 & $105.3^{*}$ & 0.4 & & & & \\
\hline $15382+3615$ & HU1167AB & 9731 & 2016.520 & $\mathrm{R}$ & 20 & 1.363 & 0.009 & 79.9 & 0.3 & & Dru1995 & 0.11 & -0.8 \\
\hline $15382+3615$ & STF1964CD & 9731 & 2016.520 & $\mathrm{R}$ & 20 & 1.563 & 0.008 & 20.3 & 0.3 & & Dru1995 & 0.06 & 0.7 \\
\hline $15390+2545$ & COU612 & - & 2016.501 & $\mathrm{~V}$ & 20 & 0.285 & 0.006 & 341.7 & 0.5 & & Doc2011h & 0.01 & $1.3^{\mathrm{Q}}$ \\
\hline
\end{tabular}


Table 2 Table of speckle measurements and O-C residuals with published orbits (cont.)

\begin{tabular}{|c|c|c|c|c|c|c|c|c|c|c|c|c|c|}
\hline WDS & Name & ADS & Epoch & Fil. & $\begin{array}{l}\text { Eyep. } \\
(\mathrm{mm})\end{array}$ & $\begin{array}{c}\rho \\
\left({ }^{\prime \prime}\right)\end{array}$ & $\begin{array}{l}\sigma_{\rho} \\
\left({ }^{\prime \prime}\right)\end{array}$ & $\begin{array}{c}\theta \\
\left(^{\circ}\right)\end{array}$ & $\begin{array}{l}\sigma_{\theta} \\
\left(^{\circ}\right)\end{array}$ & Notes & Orbit & $\begin{array}{r}\Delta \rho(\mathrm{O}-\mathrm{C}) \\
\left({ }^{\prime \prime}\right)\end{array}$ & $\begin{array}{r}\Delta \theta(\mathrm{O}-\mathrm{C}) \\
\left({ }^{\circ}\right)\end{array}$ \\
\hline $15404+2123$ & HU579 & 9741 & 2016.326 & $\mathrm{R}$ & 20 & 0.712 & 0.004 & $136.4^{*}$ & ${ }^{*} 0.3$ & & & & \\
\hline $15405+1840$ & A 2076 & 9742 & 2016.526 & $\mathrm{R}$ & 20 & 0.722 & 0.005 & 185.9 & 1.0 & Elongated & Zir2014a & -0.01 & 0.3 \\
\hline $15413+5959$ & STF1969 & 9756 & 2016.526 & $\mathrm{R}$ & 20 & 1.069 & 0.006 & 29.6 & 0.3 & & RAO2015 & 0.00 & 0.5 \\
\hline $15428+0509$ & A1125 & 9755 & 2016.326 & $\mathrm{R}$ & 20 & 1.278 & 0.006 & $278.4^{*}$ & ${ }^{*} 0.3$ & Elongated & & & \\
\hline $15440+2220$ & COU106 & - & 2016.526 & $\mathrm{R}$ & 20 & 0.331 & 0.012 & 276.8 & 1.0 & & & & \\
\hline $15468+1905$ & A2077 & 9783 & 2016.419 & $\mathrm{R}$ & 20 & 0.536 & 0.008 & 224.4 & 0.6 & & & & \\
\hline $15474+5929$ & A1127 & 9794 & 2016.302 & $\mathrm{R}$ & 20 & 0.351 & 0.006 & $296.2^{*}$ & ${ }^{*} 0.6$ & & & & \\
\hline $15476+5523$ & BU946 & 9793 & 2016.531 & $\mathrm{R}$ & 20 & 2.324 & 0.012 & 128.5 & 0.3 & & & & \\
\hline $15485+2600$ & COU616 & - & 2016.542 & $\mathrm{R}$ & 20 & 0.787 & 0.005 & 162.9 & 0.8 & & & & \\
\hline $15498+4431$ & BU621 & 9802 & 2016.414 & $\mathrm{R}$ & 20 & 0.681 & 0.005 & 25.4 & 0.5 & & & & \\
\hline $15498+4431$ & BU621 & 9802 & 2016.528 & $\mathrm{R}$ & 20 & 0.672 & 0.004 & 21.7 & 1.0 & Diffuse & & & \\
\hline $15509+1911$ & A 2078 & 9809 & 2016.528 & $\mathrm{R}$ & 20 & 1.082 & 0.014 & 165.4 & 0.3 & & & & \\
\hline $15510+4228$ & BU810 & 9814 & 2016.493 & $\mathrm{R}$ & 20 & 1.615 & 0.010 & 84.2 & 0.3 & & & & \\
\hline $15521+0528$ & A1128 & 9818 & 2016.542 & $\mathrm{R}$ & 20 & 1.530 & 0.009 & 350.1 & 0.3 & & & & \\
\hline $15530-0218$ & RST5035 & - & 2016.417 & $\mathrm{R}$ & 20 & 0.486 & 0.007 & 113.3 & 0.5 & & & & \\
\hline $15532-0116$ & RST5036 & - & 2016.329 & $\mathrm{R}$ & 10 & 0.260 & 0.002 & 22.6 & 0.6 & & & & \\
\hline $15532-0116$ & RST5036 & - & 2016.329 & $\mathrm{R}$ & 20 & 0.257 & 0.004 & 19.4 & 1.6 & & & & \\
\hline $15542+1659$ & A2080 & 9831 & 2016.417 & $\mathrm{R}$ & 20 & 0.384 & 0.004 & 103.6 & 0.4 & & Hrt2008 & 0.00 & -3.0 \\
\hline $15559+1555$ & ALD12 & 9845 & 2016.419 & $\mathrm{R}$ & 20 & 1.888 & 0.009 & 47.7 & 0.3 & & & & \\
\hline $15574+1527$ & ALD3 & 9857 & 2016.419 & $\mathrm{R}$ & 20 & 1.673 & 0.008 & 30.2 & 0.3 & & & & \\
\hline $16009+1316$ & STT303AB & 9880 & 2016.515 & $\mathrm{R}$ & 20 & 1.601 & 0.008 & 173.8 & 0.3 & Elongated & Zir2015a & 0.00 & 0.1 \\
\hline $16013+4529$ & A1640 & 9898 & 2016.509 & $\mathrm{R}$ & 20 & 0.659 & 0.011 & 345.2 & 0.7 & & & & \\
\hline $16027+1257$ & HEI240 & - & 2016.340 & $\mathrm{R}$ & 20 & 1.262 & 0.008 & 116.8 & 0.3 & & & & \\
\hline $16059+1041$ & $\mathrm{HDS} 2273 \mathrm{Aa}, \mathrm{Ab}$ & - & 2016.515 & $\mathrm{R}$ & 20 & 0.324 & 0.007 & 71.4 & 2.5 & & Zir2008 & -0.04 & -1.0 \\
\hline $16071+1654$ & BU812 & 9925 & 2016.528 & $\mathrm{R}$ & 20 & 0.709 & 0.004 & 97.5 & 0.5 & & & & \\
\hline $16115+1507$ & A1799 & 9952 & 2016.529 & $\mathrm{R}$ & 20 & 0.803 & 0.006 & 116.3 & 0.3 & & Zir2014a & 0.01 & 1.1 \\
\hline $16118+3424$ & STT306 & 9961 & 2016.501 & $\mathrm{R}$ & 10 & 0.222 & 0.004 & 2.0 & 1.3 & & & & \\
\hline $16128+3922$ & STF 2028 & 9970 & 2016.417 & $\mathrm{R}$ & 20 & 0.491 & 0.009 & 146.5 & 0.3 & & Hrt2014b & 0.01 & 0.2 \\
\hline $16137+4638$ & A1642 & 9975 & 2016.504 & $\mathrm{R}$ & 20 & 0.824 & 0.004 & 181.0 & 0.3 & & Hrt2001b & 0.04 & 0.9 \\
\hline $16139+0123$ & RST5407 & - & 2016.417 & $\mathrm{R}$ & 20 & 0.955 & 0.006 & 221.7 & 0.3 & & & & \\
\hline $16148+1600$ & A2083 & 9977 & 2016.498 & $\mathrm{R}$ & 20 & 1.188 & 0.006 & 155.6 & 0.3 & & & & \\
\hline $16161+2936$ & A348 & 9986 & 2016.378 & $\mathrm{R}$ & 20 & 1.072 & 0.008 & $151.9^{*}$ & ${ }^{*} 0.3$ & Elon & & & \\
\hline $16169+0113$ & A2181 & 9989 & 2016.417 & $\mathrm{R}$ & 20 & 0.517 & 0.004 & 85.9 & 0.5 & Elongated & Hei1996 & 0.07 & 2.7 \\
\hline $16169+1948$ & COU107 & - & 2016.417 & $\mathrm{R}$ & 20 & 0.611 & 0.004 & 113.9 & 0.4 & Elongated & & & \\
\hline $16178+4918$ & HU661 & 9994 & 2016.509 & $\mathrm{R}$ & 20 & 0.771 & 0.004 & 39.3 & 0.6 & & & & \\
\hline $16186+1247$ & HEI241 & - & 2016.378 & $\mathrm{R}$ & 20 & 0.751 & 0.004 & 58.0 & 0.3 & & & & \\
\hline $16188+1724$ & STF2037 & 9997 & 2016.414 & $\mathrm{R}$ & 20 & 1.169 & 0.006 & 255.4 & 0.3 & & & & \\
\hline $16192+4140$ & STT309 & 10006 & 2016.515 & $\mathrm{R}$ & 20 & 0.270 & 0.012 & 316.8 & 1.1 & Confused & USN2002 & -0.01 & 2.9 \\
\hline $16192+5736$ & A1137 & 10011 & 2016.376 & $\mathrm{R}$ & 20 & 0.573 & 0.004 & 203.2 & 0.8 & & & & \\
\hline $16216+3631$ & COU982 & - & 2016.509 & $\mathrm{R}$ & 20 & 0.360 & 0.008 & 247.4 & 0.5 & & & & \\
\hline $16233+3251$ & COU619 & - & 2016.376 & $\mathrm{R}$ & 20 & 0.490 & 0.004 & $190.7^{*}$ & * 0.9 & & & & \\
\hline $16235+3321$ & BU951AB,C & 10036 & 2016.501 & $\mathrm{R}$ & 20 & 1.032 & 0.005 & 29.6 & 0.5 & Elongated & & & \\
\hline $16237+0710$ & TDS810 & - & 2016.542 & $\mathrm{R}$ & 20 & 1.181 & 0.012 & 76.0 & 0.3 & & & & \\
\hline $16280+2632$ & BU813 & 10071 & 2016.414 & $\mathrm{R}$ & 20 & 1.194 & 0.006 & 176.8 & 0.3 & & & & \\
\hline $16289+1825$ & STF2052AB & 10075 & 2016.417 & $\mathrm{R}$ & 20 & 2.391 & 0.012 & 118.9 & 0.3 & Elongated & PRU2017 & -0.01 & 0.1 \\
\hline $16296+1635$ & A2084 & 10078 & 2016.379 & $\mathrm{R}$ & 20 & 0.431 & 0.008 & 132.0 & 0.5 & Elongated & & & \\
\hline $16301+3353$ & HU1173 & 10085 & 2016.509 & $\mathrm{R}$ & 20 & 0.244 & 0.006 & 66.3 & 2.9 & Elongated & Hei1997 & -0.02 & -0.3 \\
\hline $16309+0159$ & STF2055AB & 10087 & 2016.417 & $\mathrm{R}$ & 20 & 1.402 & 0.007 & 41.9 & 0.3 & & Hei1993b & -0.02 & -0.4 \\
\hline $16309+3804$ & STF2059 & 10093 & 2016.403 & $\mathrm{R}$ & 20 & 0.265 & 0.004 & 179.2 & 0.3 & & & & \\
\hline $16312+5725$ & WOR32 & - & 2016.376 & $\mathrm{R}$ & 20 & 0.992 & 0.006 & $180.4^{*}$ & ${ }^{*} 0.3$ & & & & \\
\hline $16313+5557$ & MLR636 & - & 2016.379 & $\mathrm{R}$ & 20 & 0.569 & 0.008 & 271.6 & 0.3 & Elongated & & & \\
\hline $16317+1919$ & STF2058AB & 10099 & 2016.509 & $\mathrm{R}$ & 20 & 2.045 & 0.010 & $352.5^{*}$ & ${ }^{*} 0.3$ & & & & \\
\hline $16326+2314$ & BU817 & 10107 & 2016.523 & $\mathrm{R}$ & 20 & 0.929 & 0.005 & 328.4 & 0.4 & Elongated & & & \\
\hline $16326+4007$ & STT313 & 10111 & 2016.523 & $\mathrm{R}$ & 20 & 0.921 & 0.005 & 129.9 & 0.3 & Elongated & & & \\
\hline $16366+0844$ & TDT3 & - & 2016.417 & $\mathrm{R}$ & 20 & - & - & - & - & Unres. & & & \\
\hline
\end{tabular}


Table 2 Table of speckle measurements and O-C residuals with published orbits (cont.)

\begin{tabular}{|c|c|c|c|c|c|c|c|c|c|c|c|c|c|}
\hline WDS & Name & ADS & Epoch & Fil. & $\begin{array}{l}\text { Eyep. } \\
\text { (mm) }\end{array}$ & $\underset{\rho}{\rho}\left({ }^{\prime \prime}\right)$ & $\begin{array}{l}\sigma_{\rho} \\
\left({ }^{\prime \prime}\right)\end{array}$ & $\begin{array}{c}\theta \\
\left(^{\circ}\right)\end{array}$ & $\begin{array}{l}\sigma_{\theta} \\
\left(^{\circ}\right)\end{array}$ & Notes & Orbit & $\begin{array}{r}\Delta \rho(\mathrm{O}-\mathrm{C}) \\
\left({ }^{\prime \prime}\right)\end{array}$ & $\begin{array}{r}\Delta \theta(\mathrm{O}-\mathrm{C}) \\
\left({ }^{\circ}\right)\end{array}$ \\
\hline $16368+2632$ & COU489 & - & 2016.376 & $\mathrm{R}$ & 20 & 0.732 & 0.008 & $332.0^{*}$ & 0.4 & & & & \\
\hline $16384+3514$ & COU985 & - & 2016.504 & $\mathrm{R}$ & 20 & 0.334 & 0.005 & 81.3 & 1.9 & & Doc2012g & -0.03 & 0.4 \\
\hline $16395+2244$ & HU486 & 10145 & 2016.379 & $\mathrm{R}$ & 20 & 1.315 & 0.015 & 165.1 & 0.4 & & & & \\
\hline $16421+2151$ & HU487 & 10165 & 2016.419 & V & 10 & 0.205 & 0.004 & 95.8 & 0.3 & & & & \\
\hline $16439+4329$ & D15 & 10188 & 2016.387 & $\mathrm{R}$ & 20 & 0.567 & 0.004 & 336.0 & 0.3 & & Alz2007 & 0.02 & 0.8 \\
\hline $16440+1236$ & HEI544 & - & 2016.387 & $\mathrm{R}$ & 20 & 2.923 & 0.030 & 140.2 & 0.3 & & & & \\
\hline $16468+0821$ & $\mathrm{~A} 2784 \mathrm{AB}$ & 10206 & 2016.389 & $\mathrm{R}$ & 20 & 0.388 & 0.004 & 168.5 & 0.5 & & & & \\
\hline $16497+5816$ & MLR430 & - & 2016.389 & $\mathrm{R}$ & 10 & - & - & - & - & Unres. & & & \\
\hline $16497+5816$ & MLR430 & - & 2016.389 & V & 10 & 0.131 & 0.002 & $159.0^{*}$ & 0.3 & & Lin2019 & 0.00 & -0.2 \\
\hline $16509+4259$ & A 575 & 10233 & 2016.419 & $\mathrm{R}$ & 20 & 0.699 & 0.014 & 150.4 & 2.0 & & & & \\
\hline $16511+0924$ & STF2106AB & 10229 & 2016.523 & $\mathrm{R}$ & 20 & 0.764 & 0.006 & 172.2 & 0.3 & Elongated & Sca2001g & -0.04 & 1.2 \\
\hline $16518+2840$ & STF2107AB & 10235 & 2016.490 & $\mathrm{R}$ & 20 & 1.443 & 0.007 & 104.2 & 0.3 & Elongated & Sca2003c & 0.05 & -1.5 \\
\hline $16518+3151$ & BU821 & 10236 & 2016.523 & $\mathrm{R}$ & 20 & 1.269 & 0.009 & 324.4 & 0.3 & Faint & & & \\
\hline $16522+1535$ & HU1278 & 10237 & 2016.493 & $\mathrm{R}$ & 20 & 1.395 & 0.008 & 359.4 & 0.3 & Elongated & & & \\
\hline $16539+2547$ & COU492 & - & 2016.542 & $\mathrm{R}$ & 20 & 0.515 & 0.006 & 92.1 & 0.6 & & & & \\
\hline $16540+2906$ & A350 & 10253 & 2016.600 & $\mathrm{R}$ & 20 & 0.600 & 0.012 & 148.4 & 0.3 & & & & \\
\hline $16564+3755$ & COU1287 & - & 2016.389 & V & 20 & 0.706 & 0.004 & 163.4 & 0.3 & & & & \\
\hline $16566+5711$ & $\mathrm{~A} 1143 \mathrm{AB}$ & 10276 & 2016.389 & $\mathrm{R}$ & 20 & 0.289 & 0.004 & 219.0 & 0.5 & & Hrt2000c & -0.00 & 0.7 \\
\hline $16576+4935$ & COU1772 & - & 2016.512 & $\mathrm{R}$ & 20 & 0.273 & 0.014 & 77.2 & 1.8 & Elongated & & & \\
\hline $16578+4344$ & A1872 & 10281 & 2016.542 & $\mathrm{R}$ & 20 & 1.094 & 0.009 & 119.4 & 0.3 & & & & \\
\hline $16594+1419$ & STT321 & 10294 & 2016.529 & $\mathrm{R}$ & 20 & 0.580 & 0.008 & 14.5 & 0.6 & & & & \\
\hline $16595+0942$ & BU1298AB & 10295 & 2016.512 & $\mathrm{R}$ & 20 & 0.415 & 0.008 & 132.0 & 0.3 & & & & \\
\hline $16598+3655$ & STT322 & 10302 & 2016.526 & $\mathrm{R}$ & 20 & 1.445 & 0.013 & 204.3 & 0.9 & Faint & & & \\
\hline $17007+3951$ & COU1290 & - & 2016.542 & $\mathrm{R}$ & 20 & 0.711 & 0.004 & 28.5 & 0.4 & & & & \\
\hline $17020+0827$ & STF2114 & 10312 & 2016.490 & $\mathrm{R}$ & 20 & 1.350 & 0.013 & 196.9 & 0.3 & & & & \\
\hline $17053+5428$ & STF2130AB & 10345 & 2016.529 & $\mathrm{R}$ & 20 & 2.528 & 0.013 & 1.6 & 0.6 & & Pru2012 & 0.00 & 0.1 \\
\hline $17063+2631$ & A 228 & 10346 & 2016.512 & $\mathrm{R}$ & 20 & 0.575 & 0.006 & 7.6 & 0.6 & & & & \\
\hline $17102-0046$ & BU124 & 10376 & 2016.529 & $\mathrm{R}$ & 20 & 0.907 & 0.005 & 260.7 & 0.3 & & & & \\
\hline $17116+3916$ & HU1178AB & 10391 & 2016.499 & $\mathrm{R}$ & 20 & 0.335 & 0.004 & 8.1 & 0.4 & & & & \\
\hline $17121+4540$ & KUI79AB & - & 2016.526 & $\mathrm{R}$ & 20 & 0.270 & 0.020 & 146.4 & 3.3 & & Hrt1996a & -0.04 & 1.1 \\
\hline $17130+0745$ & STT325 & 10398 & 2016.526 & $\mathrm{R}$ & 20 & 0.404 & 0.009 & 300.6 & 1.3 & & Fmr2012h & 0.05 & 2.1 \\
\hline $17141+5608$ & STT327 & 10425 & 2016.512 & $\mathrm{R}$ & 20 & 0.448 & 0.004 & 337.3 & 0.4 & & Msn2011c & 0.01 & 1.7 \\
\hline $17171+4034$ & COU1295 & - & 2016.499 & $\mathrm{R}$ & 20 & 0.260 & 0.008 & 56.4 & 1.2 & & & & \\
\hline $17174+1113$ & HU172 & 10441 & 2016.542 & $\mathrm{R}$ & 20 & 1.159 & 0.010 & 345.1 & 0.4 & & & & \\
\hline $17183+5338$ & SWI3 & 10469 & 2016.512 & $\mathrm{R}$ & 20 & 0.491 & 0.004 & 179.8 & 0.9 & & & & \\
\hline $17184+3240$ & BU628 & 10459 & 2016.512 & $\mathrm{R}$ & 20 & 0.551 & 0.004 & 261.9 & 0.7 & & Fmr2012a & -0.01 & -1.1 \\
\hline $17212+2542$ & A232 & 10495 & 2016.523 & $\mathrm{R}$ & 20 & 0.418 & 0.025 & 129.8 & 2.6 & & & & \\
\hline $17234+4209$ & STF2163 & 10523 & 2016.602 & $\mathrm{R}$ & 20 & 1.407 & 0.010 & 76.4 & 0.3 & Elongated & & & \\
\hline $17239+3627$ & STF2162 & 10527 & 2016.611 & $\mathrm{R}$ & 20 & 1.346 & 0.007 & 284.8 & 0.3 & & & & \\
\hline $17240+3835$ & HU1179 & 10531 & 2016.602 & $\mathrm{R}$ & 10 & 0.293 & 0.005 & 271.7 & 0.5 & Elongated & Hrt2000b & 0.03 & 2.5 \\
\hline $17251+0716$ & A1149 & 10533 & 2016.387 & $\mathrm{R}$ & 20 & 1.022 & 0.011 & 125.3 & 0.5 & Elongated & & & \\
\hline $17268+2625$ & COU1455AB & - & 2016.501 & $\mathrm{R}$ & 20 & 0.274 & 0.008 & $358.3^{*}$ & 2.0 & Elongated & & & \\
\hline $17276+2624$ & COU498 & - & 2016.501 & $\mathrm{R}$ & 20 & 0.457 & 0.005 & 39.8 & 1.0 & & & & \\
\hline $17291+1727$ & DAL14AB & - & 2016.529 & $\mathrm{R}$ & 20 & - & - & - & - & Unres. & & & \\
\hline $17293+2924$ & A351AB & 10585 & 2016.510 & $\mathrm{R}$ & 20 & 0.479 & 0.005 & 57.6 & 0.6 & & Hei1 & 0.02 & -1.1 \\
\hline $17304-0104$ & STF2173AB & 10598 & 2016.616 & $\mathrm{R}$ & 20 & 0.643 & 0.007 & 142.5 & 0.6 & Elongated & Hei1994a & 0.00 & 0.0 \\
\hline $17309-0538$ & A30AB & 10600 & 2016.616 & $\mathrm{R}$ & 20 & 0.462 & 0.007 & 65.8 & 1.1 & & & & \\
\hline $17320+0249$ & STT331AB & 10614 & 2016.611 & $\mathrm{R}$ & 20 & 1.013 & 0.006 & 352.6 & 0.3 & Elongated & & & \\
\hline $17327+5924$ & MLR355 & - & 2016.616 & $\mathrm{R}$ & 20 & 0.672 & 0.009 & 208.7 & 0.3 & & & & \\
\hline $17344+4913$ & HU923 & 10646 & 2016.540 & $\mathrm{R}$ & 20 & 1.015 & 0.008 & 107.3 & 0.4 & & & & \\
\hline $17345+3935$ & COU1298 & - & 2016.510 & $\mathrm{R}$ & 20 & 0.281 & 0.004 & 248.7 & 1.5 & & & & \\
\hline $17354+1322$ & A1879 & 10648 & 2016.616 & $\mathrm{R}$ & 20 & 0.520 & 0.004 & 112.6 & 0.7 & & & & \\
\hline $17374+1233$ & BU1121 & 10669 & 2016.523 & $\mathrm{R}$ & 20 & 0.483 & 0.005 & 192.1 & 1.4 & & & & \\
\hline $17399+0748$ & HDS2499 & - & 2016.611 & $\mathrm{R}$ & 20 & 0.695 & 0.007 & 242.6 & 0.8 & Elongated & & & \\
\hline $17400-0038$ & BU631 & 10696 & 2016.611 & $\mathrm{R}$ & 10 & 0.281 & 0.003 & 79.9 & 0.9 & & Hei1996a & -0.03 & 1.2 \\
\hline
\end{tabular}


Table 2 Table of speckle measurements and O-C residuals with published orbits (cont.)

\begin{tabular}{|c|c|c|c|c|c|c|c|c|c|c|c|c|c|}
\hline WDS & Name & ADS & Epoch & Fil. & $\begin{array}{l}\text { Eyep. } \\
\text { (mm) }\end{array}$ & $\begin{array}{c}\rho \\
\left({ }^{\prime \prime}\right)\end{array}$ & $\begin{array}{l}\sigma_{\rho} \\
\left({ }^{\prime \prime}\right)\end{array}$ & $\begin{array}{c}\theta \\
\left({ }^{\circ}\right)\end{array}$ & $\begin{array}{l}\sigma_{\theta} \\
\left(^{\circ}\right)\end{array}$ & Notes & Orbit & $\begin{array}{r}\Delta \rho(\mathrm{O}-\mathrm{C}) \\
\left({ }^{\prime \prime}\right)\end{array}$ & $\begin{array}{r}\Delta \theta(\mathrm{O}-\mathrm{C}) \\
\left({ }^{\circ}\right)\end{array}$ \\
\hline $17412+4139$ & STF2203 & 10722 & 2016.630 & $\mathrm{R}$ & 20 & 0.744 & 0.007 & 292.4 & 0.3 & & & & \\
\hline $17420+1557$ & BU1251AB & 10723 & 2016.630 & $\mathrm{RL}$ & 20 & 1.426 & 0.012 & 101.7 & 0.4 & Faint & Baz1991a & 0.03 & 3.4 \\
\hline $17420+1557$ & BU1251AB & 10723 & 2016.630 & $\mathrm{R}$ & 20 & 1.448 & 0.008 & 101.4 & 0.3 & & Baz1991a & 0.05 & 3.1 \\
\hline $17430+1215$ & HU1283 & 10733 & 2016.616 & $\mathrm{R}$ & 20 & 0.497 & 0.009 & 186.8 & 0.5 & & & & \\
\hline $17434+3357$ & HO560AB & 10742 & 2016.632 & $\mathrm{R}$ & 20 & 1.372 & 0.007 & 263.5 & 0.3 & & & & \\
\hline $17436+1257$ & HU1284 & 10740 & 2016.616 & $\mathrm{R}$ & 20 & 1.034 & 0.005 & 70.6 & 0.3 & & & & \\
\hline $17436+2237$ & HU1285 & 10743 & 2016.632 & $\mathrm{R}$ & 20 & 0.526 & 0.004 & 210.8 & 0.8 & & USN2002 & 0.04 & -0.6 \\
\hline $17449+5003$ & TDT485 & - & 2016.523 & $\mathrm{R}$ & 20 & - & - & - & - & Unres. & & & \\
\hline $17457+1650$ & A2092 & 10770 & 2016.643 & $\mathrm{R}$ & 32 & 0.874 & 0.019 & 338.0 & 0.4 & & Cve2008a & -0.07 & 0.5 \\
\hline $17457+1743$ & STF2205 & 10769 & 2016.633 & $\mathrm{R}$ & 20 & 0.902 & 0.006 & 11.1 & 0.4 & Elongated & & & \\
\hline $17467+1249$ & ROE141 & 10785 & 2016.643 & $\mathrm{~W}$ & 32 & 2.399 & 0.012 & 179.9 & 0.3 & & & & \\
\hline $17469+0630$ & A1162AB & 10787 & 2016.643 & $\mathrm{R}$ & 32 & 1.825 & 0.017 & 163.5 & 0.3 & & & & \\
\hline $17471+1742$ & STF2215 & 10795 & 2016.641 & $\mathrm{R}$ & 20 & 0.473 & 0.004 & 248.3 & 0.9 & Elongated & Cve2006e & 0.03 & 1.4 \\
\hline $17471+4215$ & A697 & 10800 & 2016.633 & $\mathrm{R}$ & 20 & 0.422 & 0.006 & 127.9 & 0.3 & & & & \\
\hline $17472+1502$ & HU1288 & 10796 & 2016.602 & $\mathrm{R}$ & 20 & 0.421 & 0.009 & 163.0 & 0.4 & & Zir2015a & -0.02 & 2.4 \\
\hline $17472+1502$ & HU1288 & 10796 & 2016.641 & $\mathrm{R}$ & 20 & 0.435 & 0.006 & 162.9 & 0.8 & & Zir2015a & -0.00 & 2.3 \\
\hline $17479+4047$ & TDT509 & - & 2016.641 & $\mathrm{~W}$ & 20 & - & - & - & - & Unres. & & & \\
\hline $17501+0214$ & A 2187 & 10822 & 2016.643 & $\mathrm{R}$ & 20 & 0.505 & 0.009 & 322.7 & 0.4 & Elongated & & & \\
\hline $17506+0714$ & STT337 & 10828 & 2016.654 & $\mathrm{R}$ & 20 & 0.585 & 0.004 & 164.9 & 0.4 & & Doc $2015 f$ & 0.00 & 0.7 \\
\hline $17516+4555$ & A1883 & 10855 & 2016.657 & $\mathrm{R}$ & 20 & 0.538 & 0.004 & 43.6 & 0.7 & & & & \\
\hline $17519+0724$ & A1164AB & 10846 & 2016.602 & $\mathrm{R}$ & 20 & 0.431 & 0.012 & 41.6 & 1.0 & & & & \\
\hline $17520+1520$ & STT338AB & 10850 & 2016.654 & $\mathrm{R}$ & 20 & 0.829 & 0.004 & 163.7 & 0.3 & & Pru2012 & -0.00 & 0.0 \\
\hline $17531+4212$ & COU1599 & - & 2016.654 & $\mathrm{R}$ & 20 & 0.552 & 0.006 & 134.5 & 0.4 & & & & \\
\hline $17536+4234$ & COU1600 & - & 2016.657 & $\mathrm{R}$ & 20 & 0.669 & 0.008 & 250.8 & 0.8 & & & & \\
\hline $17554+1352$ & HEI553 & - & 2016.657 & $\mathrm{~W}$ & 20 & 0.626 & 0.005 & 207.4 & 0.8 & & & & \\
\hline $17555+4108$ & COU1601Aa,Ab & 10901 & 2016.657 & $\mathrm{R}$ & 20 & 0.533 & 0.004 & 62.9 & 0.9 & & & & \\
\hline $18054+1624$ & A2093 & 11050 & 2016.493 & $\mathrm{R}$ & 20 & 0.645 & 0.008 & 229.8 & 0.3 & & & & \\
\hline $18058+2127$ & STT341AB & 11060 & 2016.515 & $\mathrm{R}$ & 10 & 0.237 & 0.004 & 92.7 & 1.1 & Diffuse & Hei1982b & -0.01 & -2.6 \\
\hline $18058+4054$ & TDT677 & - & 2016.646 & $\mathrm{~W}$ & 20 & - & - & - & - & Unres. & & & \\
\hline $18075+1940$ & STT524 & 11080 & 2016.493 & $\mathrm{R}$ & 20 & 0.478 & 0.005 & 206.5 & 1.1 & & Hrt2000c & 0.00 & 0.2 \\
\hline $18086+1838$ & HU314 & 11098 & 2016.515 & $\mathrm{R}$ & 20 & 0.271 & 0.006 & 70.9 & 2.2 & Elongated & Zir2015a & -0.02 & -1.0 \\
\hline $18092+3129$ & COU812 & - & 2016.646 & W & 20 & 0.652 & 0.005 & 264.7 & 0.4 & Elongated & Cou1999 & -0.03 & -22.6 \\
\hline $18094+3144$ & $\mathrm{~A} 237 \mathrm{AB}$ & 11114 & 2016.646 & $\mathrm{R}$ & 32 & 2.330 & 0.012 & 103.2 & 0.3 & & & & \\
\hline $18101+1629$ & STF2289 & 11123 & 2016.597 & $\mathrm{R}$ & 20 & 1.216 & 0.013 & 214.9 & 1.7 & Diffuse & Hop1964 & -0.03 & -0.7 \\
\hline $18114+2519$ & A238 & 11144 & 2016.493 & $\mathrm{R}$ & 20 & 0.619 & 0.004 & 67.8 & 0.7 & Elongated & & & \\
\hline $18121+2739$ & STF2292 & 11155 & 2016.532 & $\mathrm{R}$ & 20 & 0.834 & 0.005 & 275.5 & 0.3 & & & & \\
\hline $18124+0415$ & A353 & 11153 & 2016.526 & $\mathrm{R}$ & 20 & 1.649 & 0.010 & 17.2 & 0.4 & & & & \\
\hline $18126+3836$ & BU1091 & 11170 & 2016.540 & $\mathrm{R}$ & 20 & 0.745 & 0.006 & 319.0 & 0.3 & & Zir2012b & -0.02 & 0.3 \\
\hline $18127+5446$ & MLR585 & - & 2016.515 & $\mathrm{R}$ & 20 & 0.415 & 0.006 & 335.1 & 1.0 & & & & \\
\hline $18133-0324$ & RST4583 & - & 2016.526 & $\mathrm{R}$ & 20 & 0.392 & 0.028 & 85.6 & 2.3 & & & & \\
\hline $18133+5242$ & A1376 & 11188 & 2016.515 & $\mathrm{R}$ & 20 & 0.324 & 0.004 & 22.0 & 1.3 & & & & \\
\hline $18145+3249$ & HU927 & 11207 & 2016.526 & $\mathrm{R}$ & 20 & 0.372 & 0.006 & 100.9 & 1.3 & & & & \\
\hline $18146+0011$ & STF2294 & 11186 & 2016.540 & $\mathrm{R}$ & 20 & 1.364 & 0.007 & 92.9 & 0.3 & & Sca2015b & 0.04 & -0.4 \\
\hline $18146+2335$ & HU318AB & 11194 & 2016.649 & $\mathrm{R}$ & 20 & 0.689 & 0.008 & 156.6 & 0.3 & & & & \\
\hline $18146+2335$ & GII110CD & - & 2016.649 & $\mathrm{R}$ & 32 & 0.884 & 0.012 & $301.0^{*}$ & 0.3 & & & & \\
\hline $18146+2335$ & GII110CD & - & 2016.649 & $\mathrm{~W}$ & 32 & 0.905 & 0.020 & $301.4^{*}$ & 0.4 & & & & \\
\hline $18146+2335$ & GII110CD & - & 2016.649 & $\mathrm{R}$ & 32 & 0.898 & 0.015 & $301.0^{*}$ & 0.9 & & & & \\
\hline $18146+2335$ & GII110CD & - & 2016.652 & $\mathrm{R}$ & 32 & 0.849 & 0.022 & $301.7^{*}$ & 0.5 & & & & \\
\hline $18146+2335$ & GII110CD & - & 2016.652 & W & 32 & 0.897 & 0.009 & $300.9^{*}$ & 0.3 & & & & \\
\hline $18146+2335$ & GII110CE & - & 2016.652 & $\mathrm{~W}$ & 32 & 3.498 & 0.017 & $209.8^{*}$ & 0.3 & & & & \\
\hline $18146+2335$ & GII110CE & - & 2016.652 & $\mathrm{R}$ & 32 & 3.486 & 0.017 & $210.6^{*}$ & 0.4 & & & & \\
\hline $18160+1158$ & TDT765 & - & 2016.493 & $\mathrm{R}$ & 20 & 1.001 & 0.009 & 172.4 & 0.3 & & & & \\
\hline $18171+3453$ & COU12 & - & 2016.646 & $\mathrm{~W}$ & 32 & 2.061 & 0.016 & 210.5 & 0.3 & & & & \\
\hline $18172+2640$ & A241 & 11234 & 2016.660 & $\mathrm{~W}$ & 20 & 0.668 & 0.008 & 287.7 & 0.5 & & & & \\
\hline $18173+4355$ & A577 & 11239 & 2016.652 & $\mathrm{R}$ & 20 & 0.671 & 0.007 & 306.1 & 0.6 & & & & \\
\hline
\end{tabular}


Table 2 Table of speckle measurements and O-C residuals with published orbits (cont.)

\begin{tabular}{|c|c|c|c|c|c|c|c|c|c|c|c|c|c|}
\hline WDS & Name & ADS & Epoch & Fil. & $\begin{array}{l}\text { Eyep. } \\
(\mathrm{mm})\end{array}$ & $\begin{array}{c}\rho \\
\left({ }^{\prime \prime}\right)\end{array}$ & $\begin{array}{l}\sigma_{\rho} \\
\left({ }^{\prime \prime}\right)\end{array}$ & $\begin{array}{c}\theta \\
\left({ }^{\circ}\right)\end{array}$ & $\begin{array}{l}\sigma_{\theta} \\
\left(^{\circ}\right)\end{array}$ & Notes & Orbit & $\begin{array}{r}\Delta \rho(\mathrm{O}-\mathrm{C}) \\
\left({ }^{\prime \prime}\right)\end{array}$ & $\Delta \theta(C$ \\
\hline $18191+2935$ & A 242 & 11258 & 2016.652 & $\mathrm{R}$ & 20 & 1.321 & 0.007 & 305.2 & 0.3 & & & & \\
\hline $18197+1016$ & HU197 & 11260 & 2016.501 & $\mathrm{R}$ & 20 & 0.487 & 0.005 & 55.0 & 1.1 & Elongated & Hrt2010a & -0.02 & -2 \\
\hline $18209+3220$ & ES347 & 11276 & 2016.660 & W & 20 & 1.771 & 0.009 & 64.7 & 0.4 & Elongated & & & \\
\hline $18217+5740$ & MLR536 & - & 2016.660 & W & 20 & 0.812 & 0.005 & 193.4 & 0.3 & Elongated & & & \\
\hline $18224+0957$ & HU238 & 11294 & 2016.540 & $\mathrm{R}$ & 20 & 0.885 & 0.007 & 161.9 & 0.3 & & & & \\
\hline $18229+1458$ & HU581 & 11300 & 2016.611 & $\mathrm{R}$ & 20 & 0.365 & 0.007 & 122.3 & 1.2 & & AST1991 & 0.05 & -0 \\
\hline $18233+2731$ & $\mathrm{HO} 83$ & 11313 & 2016.502 & $\mathrm{R}$ & 20 & 0.838 & 0.010 & 114.8 & 0.3 & & & & \\
\hline $18252+5659$ & MLR537 & - & 2016.502 & $\mathrm{R}$ & 20 & 0.563 & 0.008 & 55.8 & 1.0 & & & & \\
\hline $18253+4846$ & HU66AB & 11344 & 2016.600 & $\mathrm{R}$ & 10 & 0.203 & 0.002 & 197.3 & 0.7 & & USN2002 & 0.08 & \\
\hline $18253+4846$ & HU66AB & 11344 & 2016.600 & $\mathrm{R}$ & 10 & 0.202 & 0.002 & 196.2 & 0.4 & & USN2002 & 0.08 & \\
\hline $18253+4846$ & HU66BC & 11344 & 2016.600 & $\mathrm{R}$ & 10 & 0.982 & 0.005 & $25.1^{*}$ & 0.3 & Outer peaks & Nov2008b & 0.09 & -0 \\
\hline $18253+4846$ & HU66BC & 11344 & 2016.600 & $\mathrm{R}$ & 10 & 0.980 & 0.005 & $25.6^{*}$ & ${ }^{*} 0.3$ & Outer peaks & Nov2008b & 0.09 & \\
\hline $18253+4846$ & STT351AC & 11344 & 2016.600 & $\mathrm{R}$ & 10 & 0.786 & 0.004 & $26.9^{*}$ & ${ }^{*} 0.3$ & Inner peaks & & & \\
\hline $18253+4846$ & STT351AC & 11344 & 2016.600 & $\mathrm{R}$ & 10 & 0.785 & 0.004 & $27.0^{*}$ & 0.3 & Inner peaks & & & \\
\hline $18276+4926$ & COU2278 & - & 2016.611 & $\mathrm{R}$ & 32 & 1.421 & 0.012 & 14.9 & 1.0 & & & & \\
\hline $18291+0408$ & $\mathrm{~A} 581 \mathrm{AB}$ & 11387 & 2016.600 & $\mathrm{R}$ & 20 & 0.377 & 0.005 & 140.0 & 0.3 & & & & \\
\hline $18325+0036$ & RST5450 & - & 2016.523 & $\mathrm{R}$ & 20 & 0.706 & 0.009 & 253.4 & 0.5 & & & & \\
\hline $18338+1400$ & HEI395 & - & 2016.510 & $\mathrm{R}$ & 20 & 0.374 & 0.008 & 271.6 & 1.2 & Elongated & & & \\
\hline $18361+2452$ & A 249 & 11493 & 2016.510 & $\mathrm{R}$ & 20 & 1.015 & 0.005 & 278.4 & 0.3 & & & & \\
\hline $18370+1016$ & HU247 & 11502 & 2016.523 & $\mathrm{R}$ & 20 & 0.502 & 0.037 & 182.1 & 1.3 & & & & \\
\hline $18380+0800$ & A356 & 11518 & 2016.600 & $\mathrm{R}$ & 20 & 1.027 & 0.007 & 221.6 & 0.4 & & & & \\
\hline $18383+5353$ & A1378AB & 11549 & 2016.510 & $\mathrm{R}$ & 20 & 0.373 & 0.006 & 119.1 & 0.5 & & & & \\
\hline $18385+3503$ & COU1308 & - & 2016.499 & $\mathrm{R}$ & 20 & 0.407 & 0.004 & 25.5 & 0.7 & Elongated & & & \\
\hline $18386+1632$ & HO87AB & 11530 & 2016.499 & $\mathrm{R}$ & 20 & 0.455 & 0.014 & 94.1 & 1.1 & Elongated & Hrt2000c & 0.05 & \\
\hline $18390+5559$ & A1380AB & 11562 & 2016.613 & $\mathrm{R}$ & 20 & 0.968 & 0.006 & 20.0 & 0.3 & & & & \\
\hline $18406+2636$ & COU641 & - & 2016.499 & $\mathrm{R}$ & 20 & 0.548 & 0.009 & 53.1 & 0.8 & & & & \\
\hline $18408+2142$ & HU324 & 11571 & 2016.499 & $\mathrm{R}$ & 20 & 0.886 & 0.004 & 113.3 & 0.5 & & & & \\
\hline $18413+3018$ & STF2367AB & - & 2016.630 & $\mathrm{R}$ & 20 & 0.418 & 0.006 & 72.1 & 1.3 & Elongated & $\mathrm{Pbx} 2000 \mathrm{~b}$ & -0.01 & -0 \\
\hline $18425+0518$ & TDT1014 & - & 2016.543 & $\mathrm{R}$ & 20 & 0.731 & 0.006 & 338.6 & 0.5 & & & & \\
\hline $18436+0444$ & A357 & 11610 & 2016.529 & $\mathrm{R}$ & 20 & 0.543 & 0.004 & 69.7 & 0.4 & & & & \\
\hline $18437+3141$ & $\mathrm{~A} 253$ & 11623 & 2016.523 & $\mathrm{R}$ & 20 & 0.690 & 0.004 & 132.4 & 0.4 & & Baz1987d & 0.08 & -1 \\
\hline $18439+0237$ & STF2369 & 11617 & 2016.529 & $\mathrm{R}$ & 20 & 0.279 & 0.008 & 24.8 & 1.4 & Elongated & & & \\
\hline $18448+5201$ & HU755 & 11659 & 2016.543 & $\mathrm{R}$ & 20 & 0.569 & 0.006 & 124.1 & 0.3 & & & & \\
\hline $18461+1328$ & TDT1042 & - & 2016.521 & $\mathrm{R}$ & 20 & 0.666 & 0.006 & 270.8 & 0.3 & & & & \\
\hline $18462+1001$ & HEI804 & - & 2016.613 & $\mathrm{R}$ & 32 & 1.807 & 0.013 & 128.0 & 0.5 & & & & \\
\hline $18475+4926$ & BU971AB & 11698 & 2016.512 & $\mathrm{R}$ & 20 & 0.491 & 0.005 & 34.6 & 0.9 & & & & \\
\hline $18477+0916$ & HU252 & 11687 & 2016.529 & $\mathrm{R}$ & 20 & - & - & - & - & Unres. & & & \\
\hline $18499+5516$ & MLR574 & - & 2016.641 & W & 20 & 1.052 & 0.005 & 52.8 & 0.5 & & & & \\
\hline $18526+4901$ & A1384 & 11801 & 2016.641 & $\mathrm{~W}$ & 20 & 0.693 & 0.004 & 60.7 & 0.6 & & & & \\
\hline $18549+4437$ & COU1932 & - & 2016.496 & $\mathrm{R}$ & 20 & 0.868 & 0.007 & 297.2 & 0.4 & & & & \\
\hline $18550+3053$ & A258 & 11837 & 2016.513 & $\mathrm{R}$ & 20 & 0.298 & 0.004 & 235.8 & 0.9 & & Hrt2013c & -0.04 & 5 \\
\hline $18554+1331$ & BU647AB & 11839 & 2016.641 & W & 20 & 1.307 & 0.008 & 6.7 & 0.3 & Elongated & & & \\
\hline $18554+2324$ & COU511Aa,Ab & - & 2016.496 & $\mathrm{R}$ & 20 & 0.731 & 0.006 & 189.8 & 0.5 & Elongated & & & \\
\hline $18560+4414$ & STF3130AB,C & - & 2016.419 & $\mathrm{R}$ & 20 & 2.609 & 0.013 & $257.3^{*}$ & 0.3 & Elongated & & & \\
\hline $18560+4414$ & STT365AB & 11863 & 2016.419 & $\mathrm{~V}$ & 10 & 0.168 & 0.002 & 358.5 & 1.4 & Doubtful & & & \\
\hline $18562-0034$ & BU972AB & 11851 & 2016.654 & $\mathrm{R}$ & 20 & 1.091 & 0.005 & 2.2 & 0.3 & & & & \\
\hline $18564+2944$ & COU1014 & - & 2016.420 & $\mathrm{R}$ & 20 & 0.246 & 0.004 & 45.9 & 1.8 & & & & \\
\hline $18567+2123$ & HDS2684 & - & 2016.513 & $\mathrm{R}$ & 20 & 0.597 & 0.008 & 349.6 & 0.8 & Faint & & & \\
\hline $18569+1546$ & $\mathrm{~L} 42$ & 11865 & 2016.654 & $\mathrm{R}$ & 32 & - & - & - & - & Unres. & & & \\
\hline $18571+3451$ & HDS2685 & - & 2016.603 & $\mathrm{R}$ & 20 & 0.510 & 0.004 & 198.7 & 0.3 & & & & \\
\hline $18575+5814$ & STF2438 & 11897 & 2016.521 & $\mathrm{R}$ & 20 & 0.861 & 0.007 & $357.8^{*}$ & 0.4 & Elongated & Hrt2001a & 0.04 & 0 \\
\hline $18587+1936$ & HU330 & 11896 & 2016.655 & W & 20 & 0.697 & 0.005 & 32.9 & 0.6 & Elongated & & & \\
\hline $18591+1810$ & HU331 & 11904 & 2016.543 & $\mathrm{R}$ & 20 & 0.557 & 0.005 & 207.6 & 0.3 & & & & \\
\hline $18591+2230$ & HU332 & 11906 & 2016.603 & $\mathrm{R}$ & 20 & 0.526 & 0.005 & 266.9 & 0.8 & & & & \\
\hline
\end{tabular}


Table 2 Table of speckle measurements and O-C residuals with published orbits (cont.)

\begin{tabular}{|c|c|c|c|c|c|c|c|c|c|c|c|c|c|}
\hline WDS & Name & $\mathrm{ADS}$ & Epoch & Fil. & $\begin{array}{l}\text { Eyep. } \\
(\mathrm{mm})\end{array}$ & $\begin{array}{c}\rho \\
\left({ }^{\prime \prime}\right)\end{array}$ & $\begin{array}{l}\sigma_{\rho} \\
\left({ }^{\prime \prime}\right)\end{array}$ & $\begin{array}{c}\theta \\
\left(^{\circ}\right)\end{array}$ & $\begin{array}{l}\sigma_{\theta} \\
\left(^{\circ}\right)\end{array}$ & Notes & Orbit & $\begin{array}{r}\Delta \rho(\mathrm{O}-\mathrm{C}) \\
\left({ }^{\prime \prime}\right)\end{array}$ & $\Delta \theta(\mathrm{C}$ \\
\hline $19006+3951$ & COU1933 & - & 2016.603 & $\mathrm{R}$ & 20 & 0.580 & 0.011 & 194.7 & 0.4 & & & & \\
\hline $19019+1910$ & STF2437 & 11956 & 2016.521 & $\mathrm{R}$ & 20 & 0.567 & 0.004 & 4.4 & 0.5 & & Sca2008c & 0.01 & -0 \\
\hline $19021+1426$ & НО93АВ & 11958 & 2016.499 & $\mathrm{R}$ & 20 & 1.133 & 0.006 & 328.0 & 0.4 & & & & \\
\hline $19025+5142$ & TDT1193 & - & 2016.605 & $\mathrm{R}$ & 20 & - & - & - & - & Unres. & & & \\
\hline $19027-0043$ & STF2434BC & 11971 & 2016.655 & $\mathrm{R}$ & 20 & 0.720 & 0.005 & $263.6^{*}$ & 0.3 & Elongated & Mdz2017 & -0.02 & \\
\hline $19030-0423$ & RST4615 & - & 2016.655 & W & 20 & 0.716 & 0.004 & 189.0 & 0.4 & & & & \\
\hline $19030+4233$ & A589 & 11990 & 2016.543 & $\mathrm{R}$ & 20 & 0.714 & 0.008 & 5.7 & 0.3 & & & & \\
\hline $19034+0718$ & A360 & 11981 & 2016.655 & $\mathrm{~W}$ & 20 & 0.675 & 0.008 & 285.2 & 0.5 & Elongated & & & \\
\hline $19061+3549$ & COU1614 & - & 2016.496 & $\mathrm{R}$ & 20 & 0.585 & 0.009 & 121.0 & 0.3 & & & & \\
\hline $19064+3144$ & HO97AB & 12043 & 2016.543 & $\mathrm{R}$ & 20 & 0.733 & 0.007 & 16.0 & 0.3 & & & & \\
\hline $19066+2646$ & COU722 & - & 2016.605 & $\mathrm{R}$ & 20 & 1.011 & 0.006 & 334.9 & 0.5 & Faint & & & \\
\hline $19072+4451$ & A703 & 12065 & 2016.521 & $\mathrm{R}$ & 20 & 0.560 & 0.008 & 189.2 & 0.6 & Faint & & & \\
\hline $19078+3040$ & STF2465 & 12068 & 2016.605 & $\mathrm{R}$ & 20 & 1.244 & 0.006 & 248.6 & 0.3 & & & & \\
\hline $19081+3031$ & HO99 & 12078 & 2016.499 & $\mathrm{R}$ & 20 & 0.372 & 0.004 & 164.9 & 0.5 & & & & \\
\hline $19089+1914$ & HO636 & - & 2016.603 & $\mathrm{R}$ & 20 & - & - & - & - & Unres. & & & \\
\hline $19092+1152$ & STF2464 & 12095 & 2016.603 & $\mathrm{R}$ & 20 & 1.167 & 0.006 & 28.1 & 0.3 & & & & \\
\hline $19092+2656$ & $\mathrm{HO} 444$ & 12102 & 2016.605 & $\mathrm{R}$ & 20 & 1.302 & 0.007 & 66.5 & 0.3 & & & & \\
\hline $19134+4443$ & COU2519Aa,Ab & - & 2016.605 & $\mathrm{R}$ & 20 & 0.255 & 0.004 & 76.4 & 1.0 & & & & \\
\hline $19161+4407$ & COU2520 & - & 2016.633 & $\mathrm{R}$ & 32 & 0.904 & 0.009 & 233.9 & 0.7 & & & & \\
\hline $19167+2417$ & A156 & 12257 & 2016.611 & $\mathrm{R}$ & 20 & 0.239 & 0.012 & 43.7 & 1.7 & & & & \\
\hline $19178+0317$ & A2268 & 12275 & 2016.611 & $\mathrm{R}$ & 20 & 0.540 & 0.005 & 187.4 & 0.7 & & & & \\
\hline $19182+3910$ & SEI588 & 12297 & 2016.633 & $\mathrm{~W}$ & 32 & 2.211 & 0.020 & 78.5 & 0.3 & & & & \\
\hline $19216+5223$ & BU1129 & 12366 & 2016.543 & $\mathrm{R}$ & 10 & 0.281 & 0.002 & 343.7 & 0.6 & & Baz1984a & -0.04 & \\
\hline $19224+4205$ & A592 & 12374 & 2016.499 & $\mathrm{R}$ & 20 & 0.608 & 0.006 & 250.3 & 0.6 & & & & \\
\hline $19232+1320$ & HEI269 & - & 2016.611 & $\mathrm{R}$ & 20 & 0.725 & 0.004 & 10.5 & 0.3 & Elongated & & & \\
\hline $19233+0911$ & $\mathrm{~J} 2547$ & - & 2016.633 & W & 32 & 1.965 & 0.015 & 275.6 & 0.3 & & & & \\
\hline $19240+1507$ & J2964 & - & 2016.502 & $\mathrm{R}$ & 20 & 1.237 & 0.008 & 292.7 & 0.3 & & & & \\
\hline $19241+4626$ & STT373 & 12412 & 2016.633 & $\mathrm{R}$ & 20 & 1.819 & 0.009 & 232.1 & 0.3 & & & & \\
\hline $19247+5924$ & MLR220 & - & 2016.660 & $\mathrm{~W}$ & 20 & 0.756 & 0.007 & 337.0 & 0.6 & & & & \\
\hline $19255+4631$ & A709 & 12439 & 2016.502 & $\mathrm{R}$ & 20 & 0.563 & 0.004 & $65.7^{*}$ & 0.5 & & & & \\
\hline $19264+3305$ & COU1317 & - & 2016.660 & $\mathrm{R}$ & 20 & 0.443 & 0.006 & 290.7 & 0.8 & & & & \\
\hline $19264+3529$ & TDT1460 & - & 2016.660 & $\mathrm{~W}$ & 20 & - & - & - & - & Unres. & & & \\
\hline $19267+4926$ & COU2628 & - & 2016.660 & $\mathrm{R}$ & 20 & 1.119 & 0.006 & 98.1 & 0.4 & & & & \\
\hline $19268+3457$ & POP33 & - & 2016.660 & $\mathrm{~W}$ & 20 & 0.856 & 0.004 & 232.0 & 0.6 & Elongated & & & \\
\hline $19270+1606$ & A1648 & 12459 & 2016.502 & $\mathrm{R}$ & 20 & 0.818 & 0.007 & 181.2 & 0.6 & & & & \\
\hline $19276+5025$ & TDS994 & - & 2016.660 & $\mathrm{~W}$ & 20 & 1.038 & 0.006 & 161.6 & 0.5 & Elongated & & & \\
\hline $19284+1829$ & HU340 & 12496 & 2016.613 & $\mathrm{R}$ & 20 & 0.912 & 0.005 & 119.5 & 0.3 & & & & \\
\hline $19286+3711$ & HU1303AB & 12504 & 2016.613 & $\mathrm{R}$ & 20 & 0.806 & 0.008 & 303.7 & 0.3 & & & & \\
\hline $19301+5715$ & TDT1502 & - & 2016.657 & $\mathrm{~W}$ & 20 & - & - & - & - & Unres. & & & \\
\hline $19303+4932$ & ES1097 & 12541 & 2016.616 & $\mathrm{R}$ & 20 & 1.107 & 0.007 & 244.1 & 0.6 & & & & \\
\hline $19305+2714$ & A269 & 12532 & 2016.614 & $\mathrm{R}$ & 20 & 0.622 & 0.007 & 208.4 & 0.9 & & & & \\
\hline $19309+3815$ & COU2203BC & - & 2016.616 & $\mathrm{~W}$ & 20 & 1.028 & 0.005 & 300.8 & 0.5 & Elongated & & & \\
\hline $19309+5402$ & A1398 & 12566 & 2016.510 & $\mathrm{R}$ & 20 & 1.498 & 0.007 & 48.1 & 0.3 & & & & \\
\hline $19312+3147$ & TDT1517 & - & 2016.616 & $\mathrm{R}$ & 20 & 0.609 & 0.004 & 325.5 & 0.7 & & & & \\
\hline $19312+4338$ & A595 & 12560 & 2016.657 & $\mathrm{R}$ & 20 & 1.066 & 0.005 & 85.8 & 0.3 & & & & \\
\hline $19320+3849$ & A1397AB & 12578 & 2016.616 & $\mathrm{R}$ & 20 & 1.980 & 0.010 & 98.1 & 0.5 & & & & \\
\hline $19326+1203$ & HEI574 & - & 2016.526 & $\mathrm{R}$ & 20 & 0.439 & 0.004 & 150.4 & 0.4 & & & & \\
\hline $19326+4603$ & $\mathrm{~A} 714 \mathrm{AB}$ & 12592 & 2016.526 & $\mathrm{R}$ & 20 & 1.515 & 0.008 & $359.8^{*}$ & 0.3 & & & & \\
\hline $19330+0546$ & A367 & 12584 & 2016.540 & $\mathrm{R}$ & 20 & 1.010 & 0.005 & 305.4 & 0.3 & & & & \\
\hline $19333+2629$ & COU1028Aa,Ab & - & 2016.657 & $\mathrm{R}$ & 20 & 0.304 & 0.007 & 74.1 & 0.8 & & & & \\
\hline $19353+4112$ & TDT1561 & - & 2016.616 & $\mathrm{R}$ & 20 & - & - & - & - & Unres. & & & \\
\hline $19371+0819$ & STF2544AB & 12679 & 2016.515 & $\mathrm{R}$ & 20 & 1.026 & 0.005 & 181.3 & 0.3 & & & & \\
\hline $19371+4108$ & COU2406 & - & 2016.630 & $\mathrm{R}$ & 20 & 0.861 & 0.004 & 3.3 & 0.4 & & & & \\
\hline $19379+2248$ & $\mathrm{~A} 164$ & 12704 & 2016.515 & $\mathrm{R}$ & 10 & 0.228 & 0.005 & 252.5 & 1.0 & Elongated & & & \\
\hline $19387+2517$ & J1139AB & 12734 & 2016.630 & W & 20 & 1.278 & 0.006 & 216.1 & 0.4 & Elongated & & & \\
\hline $19394+2215$ & STF2556 & 12752 & 2016.611 & $\mathrm{R}$ & 20 & 0.373 & 0.005 & 343.5 & 0.4 & & Ari1999 & 0.00 & \\
\hline
\end{tabular}


Table 2 Table of speckle measurements and O-C residuals with published orbits (cont.)

\begin{tabular}{|c|c|c|c|c|c|c|c|c|c|c|c|c|c|}
\hline WDS & Name & $\mathrm{ADS}$ & Epoch & Fil. & $\begin{array}{l}\text { Eyep. } \\
(\mathrm{mm})\end{array}$ & $\begin{array}{c}\rho \\
\left({ }^{\prime \prime}\right)\end{array}$ & $\begin{array}{l}\sigma_{\rho} \\
\left({ }^{\prime \prime}\right)\end{array}$ & $\begin{array}{c}\theta \\
\left({ }^{\circ}\right)\end{array}$ & $\begin{array}{l}\sigma_{\theta} \\
\left(^{\circ}\right)\end{array}$ & Notes & Orbit & $\begin{array}{r}\Delta \rho(\mathrm{O}-\mathrm{C}) \\
\left({ }^{\prime \prime}\right)\end{array}$ & $\begin{array}{r}\Delta \theta(\mathrm{O}-\mathrm{C}) \\
\left({ }^{\circ}\right)\end{array}$ \\
\hline $19401+3943$ & COU2407 & - & 2016.630 & $\mathrm{R}$ & 20 & - & - & - & - & Unres. & & & \\
\hline $19414+4058$ & ES1669 & 12795 & 2016.646 & $\mathrm{R}$ & 32 & 1.886 & 0.018 & 155.2 & 0.3 & & & & \\
\hline $19415+2319$ & A 2789 & 12791 & 2016.630 & $\mathrm{R}$ & 20 & 0.741 & 0.006 & 279.4 & 0.7 & & & & \\
\hline $19417+3103$ & A371 & 12797 & 2016.646 & $\mathrm{R}$ & 32 & 1.845 & 0.009 & 19.4 & 0.3 & & & & \\
\hline $19418+3056$ & TDT1645 & - & 2016.646 & $\mathrm{~W}$ & 20 & 1.103 & 0.008 & 207.3 & 0.4 & Elongated & & & \\
\hline $19419+2723$ & STT382 & 12798 & 2016.515 & $\mathrm{R}$ & 10 & 0.276 & 0.006 & 322.1 & 0.3 & Diffused & & & \\
\hline $19429+2803$ & $\mathrm{~A} 273$ & 12822 & 2016.644 & $\mathrm{R}$ & 32 & 1.738 & 0.009 & 153.1 & 0.3 & & & & \\
\hline $19432+1736$ & COU323 & - & 2016.646 & $\mathrm{R}$ & 32 & 1.162 & 0.009 & 301.4 & 0.3 & & & & \\
\hline $19432+2701$ & BU1132 & 12829 & 2016.644 & $\mathrm{R}$ & 20 & 0.558 & 0.005 & 212.2 & 1.5 & Elongated & & & \\
\hline 19439-0034 & RST5142 & - & 2016.647 & $\mathrm{R}$ & 32 & 1.455 & 0.009 & 76.8 & 0.5 & & & & \\
\hline $19450+4508$ & STF2579AB & 12880 & 2016.540 & $\mathrm{R}$ & 20 & 2.723 & 0.014 & 216.3 & 0.3 & & Sca2012c & -0.02 & -0.5 \\
\hline $19453+3048$ & AG237 & 12881 & 2016.540 & $\mathrm{R}$ & 20 & 2.414 & 0.012 & 140.6 & 0.3 & & & & \\
\hline $19453+3656$ & POP121 & - & 2016.644 & $\mathrm{R}$ & 32 & 1.589 & 0.009 & 324.3 & 0.3 & & & & \\
\hline $19458+4033$ & STT385 & 12904 & 2016.715 & $\mathrm{R}$ & 20 & 1.215 & 0.006 & 50.5 & 0.3 & Elongated & & & \\
\hline $19462+2608$ & TDT1722 & - & 2016.644 & $\mathrm{~W}$ & 20 & 0.533 & 0.004 & 201.8 & 1.6 & & & & \\
\hline $19462+2917$ & TDT1721 & - & 2016.644 & $\mathrm{~W}$ & 20 & 0.728 & 0.010 & 194.6 & 1.2 & Elongated & & & \\
\hline $19470+3926$ & MLB979 & - & 2016.647 & $\mathrm{~W}$ & 32 & 3.312 & 0.020 & 24.2 & 0.4 & & & & \\
\hline $19476+3557$ & ES2182 & 12945 & 2016.652 & $\mathrm{R}$ & 32 & 1.887 & 0.013 & 43.9 & 0.4 & & & & \\
\hline $19483+3710$ & STT386 & 12965 & 2016.600 & $\mathrm{R}$ & 20 & 0.918 & 0.008 & 69.6 & 0.3 & & & & \\
\hline $19487+3519$ & STT387 & 12972 & 2016.715 & $\mathrm{R}$ & 20 & 0.477 & 0.006 & 107.4 & 0.7 & Elongated & WSI2006b & 0.00 & 0.1 \\
\hline $19489+0545$ & BU829 & 12966 & 2016.524 & $\mathrm{R}$ & 20 & 0.764 & 0.006 & 314.0 & 0.3 & & & & \\
\hline $19495+3127$ & TDT1780 & - & 2016.652 & $\mathrm{R}$ & 20 & - & - & - & - & Unres. & & & \\
\hline $19504+2409$ & COU1034 & - & 2016.524 & $\mathrm{R}$ & 20 & 0.267 & 0.011 & 208.6 & 0.6 & & & & \\
\hline $19535+2405$ & DJU4 & - & 2016.717 & $\mathrm{R}$ & 20 & 1.425 & 0.007 & 245.6 & 0.3 & Elongated & Cve2008d & 0.06 & -1.1 \\
\hline $19541+2657$ & TDT1834 & - & 2016.652 & $\mathrm{R}$ & 20 & 0.646 & 0.006 & 345.7 & 0.4 & & & & \\
\hline $19545+5727$ & MLR587 & - & 2016.717 & $\mathrm{R}$ & 20 & 1.016 & 0.008 & 348.4 & 0.6 & Elongated & & & \\
\hline $19556+4723$ & BU831 & 13144 & 2016.717 & $\mathrm{R}$ & 20 & 0.959 & 0.005 & 127.9 & 0.3 & & & & \\
\hline $19561+3845$ & A1407 & 13146 & 2016.510 & $\mathrm{R}$ & 20 & 0.991 & 0.006 & 146.9 & 0.4 & & & & \\
\hline $19569+3706$ & COU2409 & - & 2016.717 & $\mathrm{R}$ & 20 & 1.210 & 0.010 & 319.9 & 0.3 & & & & \\
\hline $19575+2018$ & $\mathrm{BU} 425 \mathrm{AB}$ & 13165 & 2016.715 & $\mathrm{R}$ & 20 & 1.355 & 0.007 & 239.5 & 0.3 & & & & \\
\hline $19576+5250$ & A1410 & 13189 & 2016.717 & $\mathrm{R}$ & 20 & 1.449 & 0.007 & 198.4 & 0.3 & & & & \\
\hline $19579+2715$ & $\mathrm{AC} 16 \mathrm{AB}$ & 13176 & 2016.720 & $\mathrm{R}$ & 20 & 0.370 & 0.004 & 232.1 & 0.9 & Elongated & & & \\
\hline $19580+0456$ & A606 & 13169 & 2016.720 & $\mathrm{R}$ & 20 & 0.577 & 0.008 & $164.6^{*}$ & 0.6 & & Sca2016c & 0.03 & 1.1 \\
\hline $19580+0456$ & A 606 & 13169 & 2016.731 & $\mathrm{R}$ & 20 & 0.575 & 0.008 & $164.2^{*}$ & 0.6 & & Sca2016c & 0.03 & 0.7 \\
\hline $19581+2003$ & TDS1027 & - & 2016.720 & $\mathrm{R}$ & 20 & 1.043 & 0.008 & 77.7 & 0.5 & & & & \\
\hline $19583+3333$ & HO582AB & 13192 & 2016.720 & $\mathrm{R}$ & 20 & 0.581 & 0.009 & 314.0 & 0.4 & & & & \\
\hline $20020+2456$ & STT395 & 13277 & 2016.597 & $\mathrm{R}$ & 20 & 0.742 & 0.004 & 127.7 & 0.3 & & Zir2013a & -0.11 & 0.9 \\
\hline $20034+3815$ & A1412 & 13311 & 2016.605 & $\mathrm{R}$ & 20 & 0.768 & 0.007 & 227.5 & 0.3 & Faint & & & \\
\hline $20102+4357$ & STT400 & 13461 & 2016.857 & $\mathrm{R}$ & 20 & 0.661 & 0.008 & $329.4^{*}$ & 0.5 & & Hei1997 & 0.01 & 0.8 \\
\hline $20113+3550$ & BU430AB & 13486 & 2016.649 & $\mathrm{R}$ & 20 & 1.158 & 0.006 & 22.7 & 0.3 & & & & \\
\hline $20114+2217$ & COU217AB & - & 2016.649 & $\mathrm{~W}$ & 20 & 1.489 & 0.009 & 342.7 & 0.3 & Elongated & & & \\
\hline $20133+1047$ & A1202 & 13533 & 2016.649 & $\mathrm{~W}$ & 20 & 0.789 & 0.006 & 124.5 & 0.3 & & & & \\
\hline $20153+2536$ & BU983AB & 13589 & 2016.638 & $\mathrm{R}$ & 20 & 0.433 & 0.008 & 190.1 & 1.2 & & & & \\
\hline $20191+3645$ & BU1206 & 13693 & 2016.676 & $\mathrm{R}$ & 32 & 2.228 & 0.011 & $354.8^{*}$ & 0.5 & & & & \\
\hline $20196+1925$ & BRT2525 & - & 2016.677 & $\mathrm{R}$ & 32 & 1.871 & 0.011 & $59.9^{*}$ & 0.3 & & & & \\
\hline $20210+4437$ & $\mathrm{~A} 725$ & 13744 & 2016.677 & $\mathrm{R}$ & 20 & 0.817 & 0.004 & 25.4 & 0.3 & Elongated & Hrt2009 & 0.04 & -0.6 \\
\hline $20216+2346$ & STF2672 & - & 2016.857 & $\mathrm{R}$ & 20 & 0.648 & 0.009 & 350.1 & 0.6 & Elongated & & & \\
\hline $20225+4613$ & A726 & 13776 & 2016.499 & $\mathrm{R}$ & 20 & 0.497 & 0.011 & 276.3 & 0.4 & & & & \\
\hline $20237+2710$ & TDS1067 & - & 2016.641 & $\mathrm{R}$ & 32 & 1.812 & 0.013 & 203.5 & 0.8 & & & & \\
\hline $20238+4146$ & COU2641 & - & 2016.641 & $\mathrm{R}$ & 20 & 0.956 & 0.011 & $98.6^{*}$ & 0.4 & & & & \\
\hline $20240+4457$ & ES1331 & 13817 & 2016.641 & $\mathrm{R}$ & 32 & 1.263 & 0.011 & 77.6 & 0.3 & & & & \\
\hline $20243+4800$ & TDT2240 & - & 2016.641 & $\mathrm{R}$ & 20 & - & - & - & - & Unres. & & & \\
\hline $20244+1213$ & WOR33Aa,Ab & 13809 & 2016.731 & $\mathrm{R}$ & 20 & 0.594 & 0.004 & 131.2 & 0.4 & Elongated & & & \\
\hline $20244+1213$ & A1209AB & 13809 & 2016.731 & $\mathrm{R}$ & 20 & 1.876 & 0.009 & 324.2 & 0.3 & Elongated & & & \\
\hline $20244+1213$ & A1209Ab,B & 13809 & 2016.731 & $\mathrm{R}$ & 20 & 2.473 & 0.012 & 321.1 & 0.3 & Elongated & & & \\
\hline
\end{tabular}


Table 2 Table of speckle measurements and O-C residuals with published orbits (cont.)

\begin{tabular}{|c|c|c|c|c|c|c|c|c|c|c|c|c|c|}
\hline WDS & Name & ADS & Epoch & Fil. & $\begin{array}{l}\text { Eyep. } \\
(\mathrm{mm})\end{array}$ & $\begin{array}{c}\rho \\
\left({ }^{\prime \prime}\right)\end{array}$ & $\begin{array}{l}\sigma_{\rho} \\
\left({ }^{\prime \prime}\right)\end{array}$ & $\begin{array}{c}\theta \\
\left({ }^{\circ}\right)\end{array}$ & $\begin{array}{l}\sigma_{\theta} \\
\left(^{\circ}\right)\end{array}$ & Notes & Orbit & $\begin{array}{r}\Delta \rho(\mathrm{O}-\mathrm{C}) \\
\left({ }^{\prime \prime}\right)\end{array}$ & $\begin{array}{r}\Delta \theta(\mathrm{O}-\mathrm{C}) \\
\left({ }^{\circ}\right)\end{array}$ \\
\hline $20248+2254$ & BRT2839AB & - & 2016.677 & $\mathrm{~W}$ & 32 & 1.512 & 0.022 & 140.3 & 0.6 & & & & \\
\hline $20248+2254$ & BRT2839AB & - & 2016.717 & $\mathrm{~W}$ & 32 & 1.521 & 0.020 & 140.4 & 0.4 & & & & \\
\hline $20248+2254$ & SCA175BC & - & 2016.677 & W & 32 & 1.137 & 0.017 & $118.2^{*}$ & 0.4 & & & & \\
\hline $20248+2254$ & SCA175BC & - & 2016.717 & $\mathrm{~W}$ & 32 & 1.121 & 0.014 & $118.8^{*}$ & 0.6 & & & & \\
\hline $20248+2254$ & SCA175AC & - & 2016.677 & $\mathrm{~W}$ & 32 & 2.602 & 0.015 & $130.8^{*}$ & 0.3 & & & & \\
\hline $20248+2254$ & SCA175AC & - & 2016.717 & $\mathrm{~W}$ & 32 & 2.596 & 0.033 & $131.2^{*}$ & 0.3 & & & & \\
\hline $20249+3404$ & A 290 & 13834 & 2016.731 & $\mathrm{R}$ & 20 & 0.497 & 0.007 & 312.4 & 0.3 & & & & \\
\hline $20251+3408$ & COU1951 & - & 2016.731 & $\mathrm{R}$ & 20 & 1.345 & 0.010 & 179.2 & 0.4 & & & & \\
\hline $20256+2504$ & A392 & 13840 & 2016.731 & $\mathrm{R}$ & 20 & 0.899 & 0.006 & 292.2 & 0.4 & & & & \\
\hline $20312+1116$ & CHR99Aa,Ab & 13946 & 2016.540 & $\mathrm{R}$ & 10 & 0.385 & 0.003 & 141.5 & 0.3 & Diffuse & & & \\
\hline $20312+1116$ & DA1BC & 13946 & 2016.540 & $\mathrm{R}$ & 10 & 0.297 & 0.002 & 183.7 & 0.6 & & Hrt2014b & -0.04 & -4.5 \\
\hline $20396+0458$ & KUI99Aa,B & - & 2016.655 & $\mathrm{R}$ & 10 & 0.350 & 0.003 & 324.2 & 0.3 & & & & \\
\hline $20402+2039$ & SCA173 & - & 2016.655 & W & 32 & 1.521 & 0.009 & 169.7 & 0.3 & New pair & & & \\
\hline $20402+2039$ & SCA173 & - & 2016.657 & W & 32 & 1.500 & 0.009 & 169.7 & 0.3 & New pair & & & \\
\hline $20421+4125$ & COU2420 & - & 2016.600 & $\mathrm{R}$ & 20 & 0.509 & 0.011 & 89.6 & 0.6 & & & & \\
\hline $20437+4733$ & A749 & 14222 & 2016.600 & $\mathrm{R}$ & 20 & 0.476 & 0.004 & 306.0 & 0.8 & & & & \\
\hline $20445+3409$ & HU690 & 14232 & 2016.600 & $\mathrm{R}$ & 20 & 0.486 & 0.006 & 280.5 & 0.4 & & & & \\
\hline $20485+0143$ & RST5471 & - & 2016.600 & $\mathrm{R}$ & 20 & 0.645 & 0.008 & 13.9 & 0.5 & & & & \\
\hline $20489-0214$ & HO142AB & 14320 & 2016.600 & $\mathrm{R}$ & 20 & 0.999 & 0.005 & 9.9 & 0.3 & & & & \\
\hline $20519+0544$ & $\mathrm{~A} 613$ & 14368 & 2016.600 & $\mathrm{R}$ & 20 & 0.640 & 0.006 & 318.8 & 0.3 & & USN2002 & 0.03 & 0.3 \\
\hline $20521+1014$ & A1212 & 14374 & 2016.600 & $\mathrm{R}$ & 20 & 0.527 & 0.004 & 11.8 & 0.8 & & & & \\
\hline $20560+3546$ & COU2134 & - & 2016.540 & $\mathrm{R}$ & 20 & 0.557 & 0.011 & 263.6 & 1.1 & Faint & & & \\
\hline $20582+1038$ & $\mathrm{~A} 1215 \mathrm{AB}$ & 14481 & 2016.611 & $\mathrm{R}$ & 20 & 0.432 & 0.008 & 208.1 & 0.9 & Elongated & & & \\
\hline $20582+1942$ & TDT2663 & - & 2016.657 & $\mathrm{R}$ & 20 & 0.852 & 0.004 & 35.7 & 0.4 & & & & \\
\hline $21009+5929$ & MLR241 & - & 2016.616 & $\mathrm{R}$ & 20 & 0.935 & 0.006 & 174.1 & 0.4 & & & & \\
\hline $21012+5953$ & TDT2696 & - & 2016.616 & $\mathrm{R}$ & 20 & 0.526 & 0.004 & 246.3 & 0.5 & & & & \\
\hline $21021+1423$ & A1687 & 14553 & 2016.660 & W & 20 & 0.780 & 0.008 & 190.3 & 0.5 & Elongated & & & \\
\hline $21037+3857$ & TDT2720 & - & 2016.660 & $\mathrm{~W}$ & 20 & - & - & - & - & Unres. & & & \\
\hline $21039+4322$ & TDT2722 & - & 2016.660 & W & 20 & - & - & - & - & Unres. & & & \\
\hline $21051-0510$ & A177 & 14608 & 2016.603 & $\mathrm{R}$ & 20 & 1.234 & 0.007 & 336.5 & 0.3 & & & & \\
\hline $21055+5340$ & BU680AB & 14626 & 2016.862 & $\mathrm{R}$ & 20 & 0.613 & 0.007 & 282.6 & 0.7 & & & & \\
\hline $21061+2801$ & COU525 & - & 2016.633 & $\mathrm{R}$ & 32 & 1.066 & 0.016 & 167.7 & 0.4 & & & & \\
\hline $21065+4823$ & BU836AB & 14647 & 2016.605 & $\mathrm{R}$ & 20 & 0.796 & 0.004 & 172.8 & 0.3 & & & & \\
\hline $21069+4314$ & TDT2759 & - & 2016.633 & W & 20 & 0.511 & 0.008 & 118.7 & 0.5 & & & & \\
\hline $21072+1434$ & HEI79AB & - & 2016.660 & $\mathrm{R}$ & 32 & 1.731 & 0.024 & $294.1^{*}$ & 0.3 & & & & \\
\hline $21072+1434$ & HEI79AB & - & 2016.717 & $\mathrm{R}$ & 32 & 1.714 & 0.009 & $294.6^{*}$ & 0.3 & & & & \\
\hline $21072+1434$ & SCA174AC & - & 2016.717 & $\mathrm{R}$ & 32 & 1.868 & 0.009 & $285.8^{*}$ & 0.3 & New pair & & & \\
\hline $21072+1434$ & SCA174BC & - & 2016.717 & $\mathrm{R}$ & 32 & 0.315 & 0.009 & $229.4^{*}$ & 1.1 & New pair & & & \\
\hline $21080+0509$ & STT527 & 14666 & 2016.603 & $\mathrm{R}$ & 20 & 0.416 & 0.011 & 113.9 & 1.4 & & Pop1995d & 0.01 & 7.4 \\
\hline $21081+2615$ & $\mathrm{~J} 1328$ & 14671 & 2016.658 & $\mathrm{R}$ & 20 & 1.021 & 0.005 & 147.8 & 0.3 & & & & \\
\hline $21092+4737$ & TDT2786 & - & 2016.633 & $\mathrm{R}$ & 20 & - & - & - & - & Unres. & & & \\
\hline $21092+5220$ & COU2694 & - & 2016.617 & $\mathrm{R}$ & 20 & 1.128 & 0.011 & 246.9 & 0.3 & & & & \\
\hline $21097+3856$ & COU1967 & - & 2016.603 & $\mathrm{R}$ & 20 & 0.841 & 0.016 & 192.0 & 0.8 & Faint & & & \\
\hline $21097+4820$ & COU2545 & - & 2016.633 & W & 20 & 0.654 & 0.005 & 71.8 & 0.7 & & & & \\
\hline $21100+4745$ & A761 & 14704 & 2016.617 & $\mathrm{R}$ & 20 & 0.381 & 0.006 & 56.2 & 0.3 & & & & \\
\hline $21118+4417$ & TDT2812 & - & 2016.633 & $\mathrm{~W}$ & 20 & - & - & - & - & & & & \\
\hline $21121+1946$ & TDT2820 & - & 2016.617 & $\mathrm{R}$ & 20 & - & - & - & - & Unres. & & & \\
\hline $21127+4900$ & COU2652 & - & 2016.606 & $\mathrm{R}$ & 20 & 0.682 & 0.010 & 339.4 & 0.3 & Faint & & & \\
\hline $21133+4655$ & A 884 & 14766 & 2016.606 & $\mathrm{R}$ & 20 & 0.438 & 0.004 & 110.9 & 1.4 & & & & \\
\hline $21139+3830$ & COU1817 & - & 2016.630 & $\mathrm{R}$ & 20 & 0.557 & 0.004 & 110.2 & 0.4 & & & & \\
\hline $21139+4400$ & COU2299 & - & 2016.606 & $\mathrm{R}$ & 20 & 0.389 & 0.007 & 33.2 & 0.5 & & & & \\
\hline $21143+4109$ & STT432 & 14778 & 2016.854 & $\mathrm{R}$ & 20 & 1.338 & 0.007 & $115.0^{*}$ & 0.3 & & & & \\
\hline
\end{tabular}


Table 2 Table of speckle measurements and O-C residuals with published orbits (cont.)

\begin{tabular}{|c|c|c|c|c|c|c|c|c|c|c|c|c|c|}
\hline WDS & Name & ADS & Epoch & Fil. & $\begin{array}{l}\text { Eyep. } \\
(\mathrm{mm})\end{array}$ & $\begin{array}{c}\rho \\
\left({ }^{\prime \prime}\right)\end{array}$ & $\begin{array}{l}\sigma_{\rho} \\
\left({ }^{\prime \prime}\right)\end{array}$ & $\begin{array}{c}\theta \\
\left({ }^{\circ}\right)\end{array}$ & $\begin{array}{l}\sigma_{\theta} \\
\left(^{\circ}\right)\end{array}$ & Notes & Orbit & $\begin{array}{r}\Delta \rho(\mathrm{O}-\mathrm{C}) \\
\left({ }^{\prime \prime}\right)\end{array}$ & $\begin{array}{r}\Delta \theta(\mathrm{O}-\mathrm{C}) \\
\left({ }^{\circ}\right)\end{array}$ \\
\hline $21153+4017$ & A1440 & 14794 & 2016.630 & $\mathrm{R}$ & 20 & 1.161 & 0.006 & 211.0 & 0.4 & Elongated & & & \\
\hline $21161+1606$ & BU1261 & 14799 & 2016.658 & $\mathrm{R}$ & 32 & 1.740 & 0.029 & 149.5 & 0.3 & & & & \\
\hline $21161+4101$ & COU2229 & - & 2016.873 & $\mathrm{R}$ & 20 & 0.770 & 0.004 & 277.8 & 0.4 & & & & \\
\hline $21167+2955$ & TDT2878 & - & 2016.630 & $\mathrm{R}$ & 20 & 0.552 & 0.008 & 10.2 & 0.4 & & & & \\
\hline $21182+4956$ & HU692 & 14840 & 2016.658 & $\mathrm{R}$ & 20 & 0.378 & 0.006 & 259.3 & 0.6 & & & & \\
\hline $21186+3430$ & HU769 & 14842 & 2016.603 & $\mathrm{R}$ & 20 & 0.893 & 0.014 & 177.9 & 0.7 & & & & \\
\hline $21196+1421$ & HU962 & 14855 & 2016.611 & $\mathrm{R}$ & 20 & 0.618 & 0.006 & 60.8 & 0.4 & & & & \\
\hline $21197+5455$ & A1694 & 14873 & 2016.630 & $\mathrm{R}$ & 20 & 0.821 & 0.005 & 92.9 & 0.5 & & & & \\
\hline $21199+5319$ & A1695 & 14876 & 2016.658 & $\mathrm{R}$ & 20 & 0.462 & 0.004 & 193.2 & 1.3 & & & & \\
\hline $21206+4215$ & LDS2511AB & - & 2016.873 & $\mathrm{R}$ & 32 & - & - & - & - & Unres. & & & \\
\hline $21210+0022$ & RST5162 & - & 2016.611 & $\mathrm{R}$ & 20 & 0.409 & 0.004 & 21.9 & 1.0 & & & & \\
\hline $21218+4309$ & STT438 & 14907 & 2016.873 & $\mathrm{R}$ & 32 & 2.230 & 0.013 & $357.1^{*}$ & ${ }^{*} 0.4$ & & & & \\
\hline $21222+5453$ & A1696 & 14923 & 2016.611 & $\mathrm{R}$ & 32 & 1.323 & 0.012 & 48.7 & 0.3 & & & & \\
\hline $21223+2906$ & COU532 & - & 2016.614 & $\mathrm{R}$ & 20 & 0.387 & 0.006 & 45.0 & 1.5 & & & & \\
\hline $21223+5734$ & $\mathrm{~A} 764 \mathrm{AB}$ & 14926 & 2016.614 & $\mathrm{R}$ & 20 & 1.258 & 0.006 & 19.4 & 0.3 & & Hei1995 & 0.28 & -5.8 \\
\hline $21227+1533$ & J851AB & 14919 & 2016.649 & $\mathrm{R}$ & 32 & 1.405 & 0.009 & 140.9 & 0.4 & & & & \\
\hline $21245+3015$ & A1219 & 14950 & 2016.614 & $\mathrm{R}$ & 32 & 1.477 & 0.016 & 312.4 & 0.4 & & & & \\
\hline $21252+1828$ & COU430AB & - & 2016.638 & $\mathrm{R}$ & 20 & 0.552 & 0.004 & 234.3 & 0.6 & & & & \\
\hline $21256+4138$ & A618 & 14967 & 2016.614 & $\mathrm{R}$ & 20 & 0.525 & 0.011 & 276.8 & 1.2 & & & & \\
\hline $21264+3909$ & A1442 & 14979 & 2016.638 & $\mathrm{R}$ & 32 & 1.249 & 0.012 & 273.9 & 0.7 & & & & \\
\hline $21264+4911$ & ES822 & 14984 & 2016.614 & $\mathrm{R}$ & 20 & 1.435 & 0.010 & 23.5 & 0.4 & & & & \\
\hline $21277+1431$ & HEI285 & - & 2016.649 & $\mathrm{~W}$ & 20 & 0.903 & 0.007 & 98.4 & 0.5 & Elongated & & & \\
\hline $21280+4305$ & HO160 & 15000 & 2015.942 & $\mathrm{R}$ & 20 & 2.030 & 0.015 & $182.0^{*}$ & * 0.3 & Elongated & & & \\
\hline $21289+1105$ & STF2799AB & 15007 & 2015.942 & $\mathrm{R}$ & 20 & 1.877 & 0.015 & 258.6 & 0.3 & Elongated & Hrt2011a & 0.00 & 0.2 \\
\hline $21305+4620$ & A768 & 15047 & 2016.630 & $\mathrm{R}$ & 20 & 0.645 & 0.012 & 334.2 & 0.3 & & & & \\
\hline $21307+2258$ & COU134 & - & 2016.630 & $\mathrm{R}$ & 20 & - & - & - & - & Unres. & & & \\
\hline $21324+2434$ & MCT12 & - & 2016.647 & W & 32 & 1.598 & 0.022 & 239.5 & 0.4 & Elongated & & & \\
\hline $21362+3003$ & BU167 & 15126 & 2016.647 & $\mathrm{R}$ & 20 & 1.693 & 0.008 & 88.8 & 0.3 & & & & \\
\hline $21362+4253$ & HO463 & 15131 & 2016.647 & $\mathrm{R}$ & 20 & 0.508 & 0.009 & 180.2 & 0.5 & Elongated & & & \\
\hline $21392+4411$ & BU1331 & 15181 & 2016.647 & $\mathrm{R}$ & 20 & 0.928 & 0.005 & 339.3 & 0.3 & & & & \\
\hline $21393+2043$ & STT445 & 15177 & 2016.726 & $\mathrm{R}$ & 20 & 1.020 & 0.006 & 120.6 & 0.3 & Elongated & & & \\
\hline $21398+3749$ & A1444 & 15193 & 2016.647 & $\mathrm{R}$ & 20 & 1.124 & 0.006 & 82.5 & 0.3 & & & & \\
\hline $21420+1856$ & LBU2Aa,Ab & - & 2016.647 & $\mathrm{R}$ & 20 & 0.561 & 0.004 & 22.1 & 0.9 & Faint & & & \\
\hline $21431+3149$ & A1222 & 15255 & 2016.720 & $\mathrm{R}$ & 20 & 0.683 & 0.007 & 351.6 & 0.3 & & & & \\
\hline $21435+2721$ & A299AB & 15260 & 2016.726 & $\mathrm{R}$ & 20 & 1.127 & 0.006 & 64.5 & 0.5 & Elongated & & & \\
\hline $21448+0300$ & BU689 & 15278 & 2016.726 & $\mathrm{R}$ & 20 & 1.771 & 0.009 & 243.6 & 0.3 & Faint & & & \\
\hline $21521+2748$ & HO171 & 15401 & 2015.939 & $\mathrm{R}$ & 20 & 0.742 & 0.015 & 340.0 & 0.5 & & & & \\
\hline $21521+2748$ & HO171 & 15401 & 2015.939 & $\mathrm{R}$ & 20 & 0.745 & 0.015 & 340.1 & 0.3 & & & & \\
\hline $21555+5232$ & STT456AB & 15460 & 2015.939 & $\mathrm{R}$ & 20 & 1.615 & 0.015 & $216.3^{*}$ & * 0.5 & Elongated & & & \\
\hline $21559+1536$ & BAT1AB & 15455 & 2016.718 & $\mathrm{R}$ & 20 & 1.071 & 0.007 & 152.1 & 0.3 & Elongated & & & \\
\hline $21565+1940$ & HU382 & 15472 & 2016.718 & $\mathrm{R}$ & 20 & 0.309 & 0.007 & 220.9 & 1.1 & & & & \\
\hline $21576+4938$ & HU772 & 15497 & 2016.718 & $\mathrm{R}$ & 20 & 0.346 & 0.006 & 333.2 & 1.0 & & & & \\
\hline $21583+5616$ & A1898 & 15505 & 2016.718 & $\mathrm{R}$ & 20 & 1.244 & 0.006 & 223.4 & 0.3 & & & & \\
\hline $22013+4515$ & $\mathrm{~A} 780 \mathrm{AB}$ & 15556 & 2016.677 & $\mathrm{R}$ & 20 & 1.522 & 0.008 & 148.3 & 0.3 & & & & \\
\hline $22013+4515$ & A780CD & 15556 & 2016.677 & W & 20 & 1.087 & 0.005 & 110.4 & 0.3 & & & & \\
\hline $22020+2651$ & HO610AB & 15564 & 2016.677 & $\mathrm{R}$ & 20 & 0.621 & 0.008 & 241.3 & 0.4 & & & & \\
\hline $22025+2612$ & A307 & 15569 & 2016.677 & $\mathrm{R}$ & 20 & 0.401 & 0.004 & 125.3 & 0.8 & & & & \\
\hline $22028-0045$ & RST5166AB & - & 2016.726 & $\mathrm{R}$ & 20 & 0.587 & 0.006 & 11.9 & 0.8 & Elongated & & & \\
\hline $22029+4439$ & BU694AB & 15578 & 2016.939 & $\mathrm{R}$ & 20 & 1.017 & 0.006 & $6.5^{*}$ & ${ }^{*} 0.3$ & Elongated & & & \\
\hline $22038-0248$ & HO469AB & 15586 & 2016.726 & $\mathrm{R}$ & 20 & 0.437 & 0.004 & 51.2 & 1.2 & & & & \\
\hline $22050+4010$ & ES1695 & 15608 & 2016.677 & W & 20 & 1.204 & 0.006 & 37.2 & 0.4 & & & & \\
\hline $22053+4308$ & COU1826 & - & 2016.677 & $\mathrm{R}$ & 20 & 0.944 & 0.005 & 176.8 & 0.3 & & & & \\
\hline $22054+3858$ & A1453 & 15613 & 2016.939 & $\mathrm{R}$ & 20 & 0.543 & 0.007 & $329.8^{*}$ & * 0.3 & Elongated & & & \\
\hline
\end{tabular}


Table 2 Table of speckle measurements and O-C residuals with published orbits (cont.)

\begin{tabular}{|c|c|c|c|c|c|c|c|c|c|c|c|c|c|}
\hline WDS & Name & $\mathrm{ADS}$ & Epoch & Fil. & $\begin{array}{l}\text { Eyep. } \\
(\mathrm{mm})\end{array}$ & $\begin{array}{c}\rho \\
\left({ }^{\prime \prime}\right)\end{array}$ & $\begin{array}{l}\sigma_{\rho} \\
\left(^{\prime \prime}\right)\end{array}$ & $\begin{array}{c}\theta \\
\left({ }^{\circ}\right)\end{array}$ & $\begin{array}{l}\sigma_{\theta} \\
\left(^{\circ}\right)\end{array}$ & Notes & Orbit & $\begin{array}{r}\Delta \rho(\mathrm{O}-\mathrm{C}) \\
\left({ }^{\prime \prime}\right)\end{array}$ & $\begin{array}{r}\Delta \theta(\mathrm{O}-\mathrm{C}) \\
\left({ }^{\circ}\right)\end{array}$ \\
\hline $22057+3521$ & PRU2 & - & 2016.939 & $\mathrm{R}$ & 20 & - & - & - & - & Unres. & & & \\
\hline $22059+4522$ & A183 & 15633 & 2016.644 & $\mathrm{R}$ & 20 & 0.728 & 0.004 & 245.5 & 0.3 & & & & \\
\hline $22061+2034$ & BRT2840 & - & 2016.726 & $\mathrm{R}$ & 32 & 2.389 & 0.016 & 316.5 & 0.4 & & & & \\
\hline $22061+4159$ & $\mathrm{~A} 407 \mathrm{AB}$ & 15636 & 2016.644 & $\mathrm{R}$ & 20 & 0.650 & 0.004 & 22.2 & 0.4 & & & & \\
\hline $22070+3605$ & STT462AB & 15645 & 2015.942 & $\mathrm{R}$ & 20 & 1.054 & 0.015 & $314.8^{*}$ & 0.3 & Elongated & & & \\
\hline $22100+2308$ & COU136 & - & 2015.942 & $\mathrm{R}$ & 20 & 0.439 & 0.015 & 18.6 & 0.5 & & Doc2016h & -0.02 & -0.9 \\
\hline $22155+5549$ & MLR611 & - & 2016.600 & $\mathrm{R}$ & 20 & 0.382 & 0.004 & 140.3 & 0.5 & & & & \\
\hline $22173+5049$ & HU595 & 15813 & 2016.600 & $\mathrm{R}$ & 20 & 0.570 & 0.004 & 221.7 & 0.8 & & & & \\
\hline $22186+5434$ & MLR612 & - & 2016.600 & $\mathrm{R}$ & 20 & 0.346 & 0.011 & 165.2 & 0.7 & & & & \\
\hline $22202+2931$ & BU1216 & 15843 & 2015.939 & $\mathrm{R}$ & 20 & 0.934 & 0.015 & $275.4^{*}$ & 0.3 & & Lin2012a & 0.05 & -1.8 \\
\hline $22206+5349$ & BU379 & 15856 & 2015.939 & $\mathrm{R}$ & 20 & 1.125 & 0.015 & $333.1^{*}$ & 0.3 & & & & \\
\hline $22234+1733$ & HO182 & 15894 & 2016.720 & $\mathrm{R}$ & 20 & 1.454 & 0.007 & 309.4 & 0.3 & & & & \\
\hline $22239+1637$ & HEI83 & - & 2016.720 & $\mathrm{R}$ & 20 & 0.759 & 0.005 & 109.1 & 0.4 & & & & \\
\hline $22247+1914$ & HU493 & 15899 & 2016.641 & W & 20 & 0.807 & 0.009 & 171.6 & 0.4 & Elongated & & & \\
\hline $22265+1925$ & HU596 & 15936 & 2016.720 & $\mathrm{R}$ & 20 & 1.139 & 0.006 & 197.2 & 0.3 & & & & \\
\hline $22281+1215$ & BU701AB & 15962 & 2016.720 & $\mathrm{R}$ & 20 & 0.975 & 0.013 & 178.6 & 0.4 & & Cve2005 & -0.01 & 0.9 \\
\hline $22303+1810$ & COU331 & - & 2016.655 & $\mathrm{~W}$ & 20 & 0.418 & 0.005 & 176.3 & 1.2 & Elongated & & & \\
\hline $22313+1304$ & BRT1361 & - & 2016.720 & $\mathrm{~W}$ & 32 & 3.352 & 0.020 & 134.8 & 0.3 & Elongated & & & \\
\hline $22314+1332$ & TDT3567 & - & 2016.655 & $\mathrm{~W}$ & 20 & - & - & - & - & Unres. & & & \\
\hline $22325+3500$ & POP39 & - & 2016.652 & $\mathrm{R}$ & 20 & 0.626 & 0.006 & $95.1^{*}$ & 0.3 & & & & \\
\hline $22333+1203$ & HEI411 & - & 2016.649 & $\mathrm{~W}$ & 20 & 1.511 & 0.013 & 301.6 & 0.3 & Elongated & & & \\
\hline $22340+4051$ & ES1697 & 16063 & 2016.655 & $\mathrm{~W}$ & 32 & 2.923 & 0.015 & 338.1 & 0.3 & & & & \\
\hline $22344+2514$ & COU141 & - & 2016.649 & $\mathrm{~W}$ & 20 & 1.513 & 0.008 & 205.6 & 0.3 & Elongated & & & \\
\hline $22344+2623$ & COU540 & - & 2016.649 & $\mathrm{R}$ & 20 & 0.340 & 0.006 & 328.0 & 1.0 & & Mnt2004a & 0.00 & 5.9 \\
\hline $22359+4515$ & GII55 & - & 2016.655 & $\mathrm{~W}$ & 32 & 5.214 & 0.026 & 311.0 & 0.3 & & & & \\
\hline $22359+4515$ & GII55 & - & 2016.658 & $\mathrm{~W}$ & 32 & 5.215 & 0.026 & 310.9 & 0.3 & & & & \\
\hline $22360+4515$ & COU2242 & - & 2016.655 & $\mathrm{~W}$ & 20 & 0.823 & 0.004 & 275.5 & 0.3 & Elongated & & & \\
\hline $22373+5420$ & MLR614 & - & 2016.614 & $\mathrm{R}$ & 20 & 0.567 & 0.008 & 307.0 & 0.7 & & & & \\
\hline $22390+1850$ & HU392AB & 16139 & 2016.652 & $\mathrm{R}$ & 20 & 0.496 & 0.004 & 346.9 & 0.6 & & & & \\
\hline $22392+2014$ & HU393 & 16142 & 2016.652 & $\mathrm{R}$ & 20 & 0.897 & 0.006 & 224.9 & 0.5 & & & & \\
\hline $22393+4000$ & MLB906 & - & 2016.652 & W & 32 & 2.582 & 0.013 & 156.0 & 0.3 & & & & \\
\hline $22397+5441$ & A1473 & 16161 & 2016.614 & $\mathrm{R}$ & 20 & 1.449 & 0.007 & 288.6 & 0.4 & & & & \\
\hline $22402+3732$ & HO188 & 16164 & 2016.715 & $\mathrm{R}$ & 20 & 0.368 & 0.004 & 229.1 & 1.4 & & USN2006b & 0.08 & -4.0 \\
\hline $22414+0443$ & BU480 & 16180 & 2016.652 & $\mathrm{R}$ & 20 & 1.065 & 0.005 & 60.4 & 0.3 & & & & \\
\hline $22426+2943$ & BU710 & 16199 & 2016.614 & $\mathrm{R}$ & 20 & 0.494 & 0.005 & 246.4 & 0.7 & & & & \\
\hline $22443+3442$ & ES2203 & 16230 & 2016.715 & $\mathrm{R}$ & 32 & 2.105 & 0.021 & 96.8 & 0.3 & & & & \\
\hline $22451-0240$ & A2696BC & 16235 & 2016.652 & $\mathrm{R}$ & 20 & 0.607 & 0.004 & 75.2 & 0.6 & Elongated & Hrt2014b & -0.01 & -1.3 \\
\hline $22451+5458$ & A1474 & 16244 & 2016.633 & $\mathrm{R}$ & 20 & 0.491 & 0.008 & 7.4 & 0.7 & & & & \\
\hline $22451+5527$ & TDT3689 & - & 2016.633 & $\mathrm{R}$ & 20 & 0.724 & 0.004 & 98.5 & 0.5 & & & & \\
\hline $22497+5007$ & COU2663 & - & 2016.633 & W & 20 & 0.631 & 0.008 & 113.0 & 0.7 & & & & \\
\hline $22508+2404$ & TDT3747 & - & 2016.715 & $\mathrm{R}$ & 20 & - & - & - & - & Unres. & & & \\
\hline $22513+2438$ & TDT3751 & - & 2016.715 & $\mathrm{R}$ & 20 & - & - & - & - & Unres. & & & \\
\hline $22541+0246$ & HO483 & 16344 & 2016.652 & $\mathrm{~W}$ & 32 & 1.705 & 0.022 & 350.4 & 0.3 & & & & \\
\hline $22557+1547$ & HU987 & 16373 & 2016.944 & $\mathrm{R}$ & 20 & 1.211 & 0.006 & $75.4^{*}$ & 0.3 & Elongated & USN2007a & 0.06 & -1.1 \\
\hline $22564+2257$ & COU240 & - & 2016.944 & $\mathrm{R}$ & 20 & 0.793 & 0.004 & $294.3^{*}$ & 0.4 & & & & \\
\hline $22574+1958$ & COU334 & - & 2016.652 & $\mathrm{R}$ & 20 & 1.126 & 0.007 & 256.1 & 0.4 & Faint & & & \\
\hline $22579+5439$ & A1477 & 16409 & 2015.939 & $\mathrm{R}$ & 20 & 0.497 & 0.015 & $352.3^{*}$ & 0.3 & & & & \\
\hline $22579+5439$ & A1477 & 16409 & 2016.857 & $\mathrm{R}$ & 20 & 0.487 & 0.007 & $353.4^{*}$ & 0.6 & & & & \\
\hline $22590+4617$ & A192 & 16430 & 2016.633 & $\mathrm{R}$ & 20 & 0.332 & 0.006 & 230.7 & 0.5 & & & & \\
\hline $22592+1144$ & STT483 & 16428 & 2016.660 & $\mathrm{R}$ & 20 & 0.457 & 0.004 & 19.7 & 0.9 & & Alz2007 & 0.02 & -4.7 \\
\hline $22597+4149$ & HLD56 & 16435 & 2015.939 & $\mathrm{R}$ & 20 & 1.188 & 0.015 & $91.3^{*}$ & 0.3 & & & & \\
\hline $23042+1518$ & TDT3871 & - & 2016.660 & $\mathrm{R}$ & 20 & - & - & - & - & Unres. & & & \\
\hline
\end{tabular}


Table 2 Table of speckle measurements and O-C residuals with published orbits (cont.)

\begin{tabular}{|c|c|c|c|c|c|c|c|c|c|c|c|c|c|}
\hline WDS & Name & ADS & Epoch & Fil. & $\begin{array}{l}\text { Eyep. } \\
(\mathrm{mm})\end{array}$ & $\begin{array}{c}\rho \\
\left({ }^{\prime \prime}\right)\end{array}$ & $\begin{array}{l}\sigma_{\rho} \\
\left({ }^{\prime \prime}\right)\end{array}$ & $\begin{array}{c}\theta \\
\left(^{\circ}\right)\end{array}$ & $\begin{array}{l}\sigma_{\theta} \\
\left({ }^{\circ}\right)\end{array}$ & Notes & Orbit & $\begin{array}{r}\Delta \rho(\mathrm{O}-\mathrm{C}) \\
\left({ }^{\prime \prime}\right)\end{array}$ & $\begin{array}{r}\Delta \theta(\mathrm{O}-\mathrm{C}) \\
\left({ }^{\circ}\right)\end{array}$ \\
\hline $23055+4643$ & A196 & 16505 & 2016.718 & $\mathrm{R}$ & 20 & 0.455 & 0.008 & 313.5 & 0.6 & & & & \\
\hline $23078+1240$ & BU1025AB & 16524 & 2016.726 & $\mathrm{R}$ & 20 & 0.795 & 0.008 & 330.6 & 0.3 & Elongated & & & \\
\hline $23096-0358$ & A311 & 16551 & 2016.726 & $\mathrm{R}$ & 20 & 1.388 & 0.007 & 145.0 & 0.3 & Elongated & & & \\
\hline $23099+4447$ & COU1844 & - & 2016.718 & $\mathrm{R}$ & 20 & 0.504 & 0.007 & 167.0 & 0.5 & & & & \\
\hline $23099+4452$ & COU2341 & - & 2016.718 & $\mathrm{R}$ & 20 & 0.895 & 0.004 & 23.0 & 0.4 & & & & \\
\hline $23101+5158$ & HU1322 & 16559 & 2016.660 & $\mathrm{R}$ & 20 & 0.490 & 0.004 & 123.2 & 0.4 & & & & \\
\hline $23102+5727$ & STT490AB & 16560 & 2016.854 & $\mathrm{R}$ & 20 & 1.185 & 0.006 & $297.6^{*}$ & 0.3 & & & & \\
\hline $23110+0637$ & HEI829AB & - & 2016.652 & W & 20 & 1.060 & 0.007 & 86.7 & 0.3 & Elongated & & & \\
\hline $23117+3730$ & A1480 & 16578 & 2016.857 & $\mathrm{R}$ & 20 & 0.759 & 0.004 & 222.7 & 0.5 & Elongated & & & \\
\hline $23147+4116$ & A200 & 16621 & 2016.854 & $\mathrm{R}$ & 20 & 0.601 & 0.004 & $76.8^{*}$ & 0.8 & & & & \\
\hline $23156+5829$ & MLR365 & - & 2016.726 & $\mathrm{R}$ & 32 & 2.093 & 0.010 & 192.7 & 0.3 & & & & \\
\hline $23195+4225$ & COU1645 & - & 2016.660 & $\mathrm{~W}$ & 20 & 0.733 & 0.006 & 76.2 & 0.7 & Diffuse & & & \\
\hline $23210+4926$ & COU2700 & - & 2016.854 & $\mathrm{R}$ & 20 & 1.032 & 0.005 & 238.1 & 0.4 & & & & \\
\hline $23237+3739$ & A1483 & 16726 & 2016.603 & $\mathrm{R}$ & 20 & 0.541 & 0.005 & 326.6 & 0.6 & & & & \\
\hline $23241+5732$ & STT495 & 16731 & 2016.603 & $\mathrm{R}$ & 20 & 0.393 & 0.006 & 123.5 & 1.2 & & Alz2006 & 0.00 & 1.4 \\
\hline $23253+5321$ & MLR619 & - & 2016.660 & $\mathrm{~W}$ & 20 & 0.894 & 0.005 & 21.2 & 0.5 & & & & \\
\hline $23260+2742$ & HO489AB & 16748 & 2016.944 & $\mathrm{R}$ & 20 & 0.512 & 0.007 & $219.0^{*}$ & 1.4 & Elongated & & & \\
\hline $23267+4103$ & COU1845 & - & 2016.658 & $\mathrm{R}$ & 20 & 0.947 & 0.008 & 0.5 & 0.6 & Elongated & & & \\
\hline $23268+5434$ & A1485 & 16760 & 2016.854 & $\mathrm{R}$ & 20 & 0.579 & 0.006 & 208.5 & 0.8 & & & & \\
\hline $23292+4042$ & A1487 & 16785 & 2016.950 & $\mathrm{R}$ & 20 & 1.112 & 0.011 & $157.0^{*}$ & 0.4 & Elongated & & & \\
\hline $23302+1359$ & HU999 & 16797 & 2016.720 & $\mathrm{R}$ & 20 & 1.573 & 0.011 & 141.9 & 0.3 & Elongated & & & \\
\hline $23312+5604$ & TDT4080 & - & 2016.644 & W & 20 & - & - & - & - & Unres. & & & \\
\hline $23318+2148$ & COU243 & - & 2016.720 & $\mathrm{R}$ & 20 & 0.897 & 0.009 & 343.5 & 0.4 & Elongated & & & \\
\hline $23334+3215$ & TDT4096 & - & 2016.677 & $\mathrm{~W}$ & 20 & 0.421 & 0.011 & 104.3 & 0.6 & Elongated & & & \\
\hline $23339+2342$ & COU144 & - & 2016.944 & $\mathrm{R}$ & 20 & 0.332 & 0.004 & 44.9 & 0.9 & & & & \\
\hline $23368+2346$ & HU498 & 16867 & 2016.677 & $\mathrm{~W}$ & 20 & 0.686 & 0.007 & 295.8 & 0.4 & Elongated & & & \\
\hline $23377+4457$ & COU2249 & - & 2016.647 & $\mathrm{R}$ & 20 & 0.488 & 0.004 & 31.5 & 0.5 & Elongated & & & \\
\hline $23379+5806$ & A642 & 16881 & 2016.644 & $\mathrm{R}$ & 20 & 0.918 & 0.005 & 24.1 & 0.3 & & & & \\
\hline $23380+1253$ & $\mathrm{~A} 1241 \mathrm{AB}$ & 16882 & 2016.677 & $\mathrm{R}$ & 20 & 0.652 & 0.004 & 11.2 & 0.4 & & & & \\
\hline $23386+1151$ & TDT4136 & - & 2016.677 & $\mathrm{~W}$ & 20 & - & - & - & - & Unres. & & & \\
\hline $23401+1258$ & HU1325 & 16914 & 2016.647 & $\mathrm{R}$ & 20 & 0.887 & 0.006 & 35.9 & 0.3 & & Sca2003a & 0.01 & 4.1 \\
\hline $23409+0759$ & BU724 & 16924 & 2016.950 & $\mathrm{R}$ & 20 & 0.620 & 0.007 & $100.4^{*}$ & 0.5 & Elongated & & & \\
\hline $23425+5436$ & A1495 & 16941 & 2016.647 & $\mathrm{R}$ & 20 & 0.484 & 0.004 & 188.6 & 0.4 & & & & \\
\hline $23432+4313$ & POP172 & - & 2016.647 & $\mathrm{R}$ & 32 & 1.314 & 0.009 & 346.2 & 0.3 & & & & \\
\hline $23440+2922$ & AGC14 & 16957 & 2016.644 & $\mathrm{R}$ & 20 & 0.837 & 0.008 & 283.9 & 0.3 & & Pop1997f & -0.06 & 0.5 \\
\hline $23447+1146$ & TDT4172 & - & 2016.720 & $\mathrm{R}$ & 20 & 0.615 & 0.005 & 75.1 & 1.3 & & & & \\
\hline $23472+5755$ & TDT4191 & - & 2016.726 & $\mathrm{R}$ & 20 & 0.254 & 0.004 & 171.7 & 0.6 & & & & \\
\hline $23492+5838$ & $\mathrm{~A} 645$ & 17026 & 2016.718 & $\mathrm{R}$ & 20 & 0.887 & 0.004 & 97.3 & 0.3 & & & & \\
\hline $23495+5628$ & MLR623 & - & 2016.718 & $\mathrm{R}$ & 20 & 0.774 & 0.006 & 116.2 & 0.3 & & & & \\
\hline $23500+4755$ & TDT4226 & - & 2016.726 & $\mathrm{R}$ & 20 & - & - & - & - & Unres. & & & \\
\hline $23504+2620$ & COU545 & - & 2016.731 & $\mathrm{R}$ & 20 & 0.722 & 0.007 & 315.9 & 0.5 & Diffuse & & & \\
\hline $23505+4703$ & A792 & 17036 & 2016.734 & $\mathrm{R}$ & 20 & 0.655 & 0.009 & 268.4 & 0.4 & & & & \\
\hline $23512+4847$ & A795 & 17046 & 2016.732 & $\mathrm{R}$ & 20 & 1.225 & 0.006 & 318.5 & 0.3 & & & & \\
\hline $23514+4745$ & A796 & 17047 & 2016.734 & $\mathrm{R}$ & 20 & 0.708 & 0.006 & 8.7 & 0.6 & & & & \\
\hline $23515+5913$ & MLR367 & - & 2016.734 & $\mathrm{R}$ & 20 & - & - & - & - & Unres. & & & \\
\hline $23536+3316$ & TDT4258 & - & 2016.734 & $\mathrm{~W}$ & 20 & - & - & - & - & Unres. & & & \\
\hline $23561+2520$ & A 426 & 17105 & 2016.649 & $\mathrm{R}$ & 20 & 0.377 & 0.004 & 283.8 & 0.3 & & Hrt2001b & -0.02 & -5.0 \\
\hline $23579+5723$ & STF3047AB & - & 2016.865 & $\mathrm{R}$ & 20 & 1.160 & 0.008 & 71.3 & 0.4 & & & & \\
\hline $23595+5441$ & A1498 & 17151 & 2016.650 & $\mathrm{R}$ & 20 & 0.375 & 0.006 & 89.5 & 0.9 & & & & \\
\hline
\end{tabular}

Note: In column 9 , the exponent ${ }^{*}$ indicates that the position angle $\theta$ could be determined without the $180^{\circ}$ ambiguity. 